\title{
MEASUREMENT OF THE LARGE-SCALE ANISOTROPY OF THE COSMIC BACKGROUND RADIATION AT $3 \mathrm{~mm}$
}

\author{
LBL- 17118 \\ Gerald Lewis Epstein \\ DE84 006774 \\ (Ph.D. Thesis) \\ Lawrence Berkeley Laboratory \\ University of California \\ Berkeley, California 94720
}

December 1983

\begin{abstract}
DISCLAIMER
This report was prepared as an account of work sponsored by an agency of the United States Government. Neither the United States Government nor any agency thereof, nor any of their employees, makes any warranty, express or implied, or assumes any legal liability or responsibility for the accuracy, conroleteness, or usefulness of any information, apparatus, product, or process disclosed, or represents that its use would not infringe privately owned rights. Reference herein to any specific commercial product, process, or sirvice by trade name, trademark, manufacturer, or otherwise does not necessarily constitute or imply its endorsement, recommendation, or favoring by the United States Governmeat or any agency thereof. The views and opinions of authors expressed herein do not necessarily state or reflect those of the United States Government or any agtisy thereof.
\end{abstract}

This research was supported by the Director, Office of Energy Research, Office of High Energy and Nuclear Physics, Division of High Energy Physics of the U.S. Department of Energy under Contract No. DE-AC03-76SF00098. 


\author{
Measurement of the Large-Scale Anisotropy \\ of the Cosmic Background Radiation at $3 \mathrm{~mm}$
}

Gerald Lewis Epsteנn

\begin{abstract}
Abstraet
A balloon-borne differential radiometer has measured the large-scal 3nisotropy of the co-mic background radiation (CBR) with high sensitivity. The antenna teuperature dipole anisotropy at $90 \mathrm{GHz}$ ( $3 \mathrm{~mm}$ wavelength) is $2.82 \pm 0.19 \mathrm{mK}$, corresponding to a thermodynamic anisotropy of $3.48 \pm 0.24 \mathrm{mK}$ for $82.7 \mathrm{~K}$ blackbody CBR. The dipole direction, $11.3 \pm 0.1$ hours right ascension and $-5.7^{\circ} \pm 1.8^{\circ}$ declination, agrees well with measurements at other frequencies. Calibration error dominates magnitude uncertainty, with statistical errors on dipole terms being under $0.1 \mathrm{mK}$. No signiffcant quadrupole power is fuund, placing a $90 \%$ confldence-level upper limit of $0.27 \mathrm{mK}$ on the RMS thermodynamic quadrupolar anisotropy.

The cryogenically-cooled receiver has a donble-sideband noise temperature of $135 \mathrm{~K}$ and a sensitivity of $12 \mathrm{mK}$ for one second of integration time. Comparing two sky regions $45^{\circ}$ from zenith and $180^{\circ}$ apart in azimuth, it can resolve the dipole anisotropy in real-time as the balloon gondola rotates. In-flight calibrations show that gain stability is better than $\pm 1 \%$. With flights from Palestine, Texas ( $31.8^{\circ}$ north latitude) in November 1981 and April 1982, the radiometer has surveyed the sky between -15 and +75 degrees declination.
\end{abstract}


Since the magnitude of the dipole anisotropy induced by motion through the CBR depends on both the CBR intensity and its spectral index, anisotropy measurements at several frequencies provide information about the CBR spectrum. The ratio of the dipole magnitudes measured at $90 \mathrm{GHz}$ by this experiment and at $24.5 \mathrm{GHz}$ by another recent observation is consistent with a $2: k$ blackbely $\mathrm{CBR}$ but does not require such a spectrum. If the CBR spectrum is blackbody ibe two measurements establish a $90 \%$ conffdence-level lower limit of $2.2 \mathrm{~K}$ (i) its tewperature.

Th: region within tive degrees of the galactic plane has been escluded from the fits. Including it changes the fltted parameters by less than one statistical slandard deviation. Fitting to galactic models indicates tbat galactic emission averaged over the region within five degrees of the plane is on the order of $0.5 \mathrm{mk}$. 
For Joel Oriof 


\section{Contents}

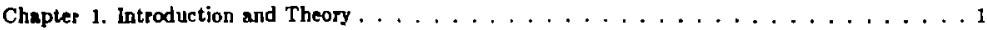

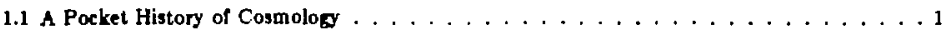

1.1.1 Early Earth-Centered Models . . . . . . . . . . . . . . . . . 1

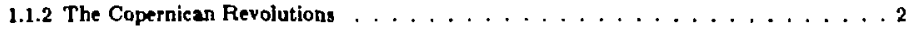

1.2 Modern Mathematical Cosmology . . . . . . . . . . . . . . . 3

1.2 .1 Ge.neral Relativity . . . . . . . . . . . . . . . . . 3

1.2 .2 Big Bang Cosmologies . . . . . . . . . . . . . . . . . . . 4

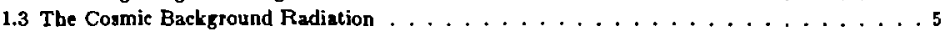

1.3.1 CBR Prediction . . . . . . . . . . . . . . . . 6

1.3 .2 Djscovery . . . . . . . . . . . . . . . . . . . . . .

1.3 .3 Properties . . . . . . . . . . . . . . . . . .

1.3.3.1 Specteum . . . . . . . . . . . . . . . . .

1.3 .3 .2 Polarization . . . . . . . . . . . . . . . 10

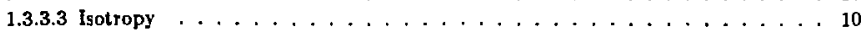

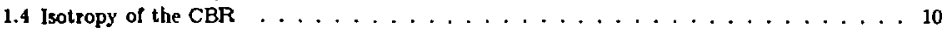

1.4.1 Isotropy of the Universe . . . . . . . . . . . . . . . 10

1.4.1.1 Chaotic Cosmology . . . . . . . . . . . . . . . . 11

1.4 .1 .2 Anthropic Principle . . . . . . . . . . . . . . 11

1.4.1.3 The Inflationary Universe . . . . . . . . . . . . . . 13

1.4.2 Non-Intringic Sources of Anjsotropy of the CBR . . . . . . . . . . . 16

1.4.2.1 Peculiar Velocity and Antenna Temperature . . . . . . . . . . 16

1.4.2.2 Gravitational Radiation . . . . . . . . . . . . 18

1.4 .3 Intrinsic Anisotropy . . . . . . . . . . . . . . . . . . 18

1.4.3.1 Anisotropic Cosmologies . . . . . . . . . . . . . 18

1.4.3.2 Inhomogeneous Matter Distribution . . . . . . . . . . . 20

1.4.4 Previous Experimental Resulty . . . . . . . . . . . . . . . 20

1.4.4.1 Ground-Based Observations . . . . . . . . . . . . 20

1.4.4.2 Aerial Observations . . . . . . . . . . . . . . 21

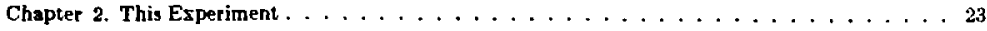

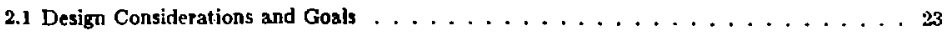

2.1.1 Choice of Frequency . . . . . . . . . . . . . . . . . . 23

2.1.2 Chnice of Technolog . . . . . . . . . . . . . . . . 25

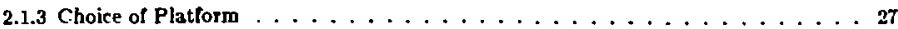


2.2 Radiometer Degcription . . . . . . . . . . . . . . . . . . . . . . 27

2.2.1 Radiometer Hardware . . . . . . . . . . . . . . . . . . . . . . . 29

2.2.1.1 Cryogenic Temperature Components . . . . . . . . . . . . . 29

2.2.1.2 Nen-Cryogenje Temperature Components . . . . . . . . . . . . . . 33

2.2.2 Support Equipment . . . . . . . . . . . . . . . . . . . 35

2.2.2.1 Magnetometers . . . . . . . . . . . . . . . . . . 35

2.2.2.2 In-Flight Calibrator . . . . . . . . . . . . . . . . 36

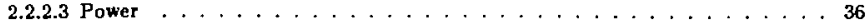

2.2.2.4 Temperature Regulation . . . . . . . . . . . . . . . . 36

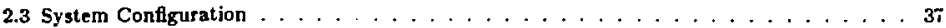

2.3.1 Experiment and Gondola . . . . . . . . . . . . . . . . . . . 37

2.3.2 Balloon and Flight Train . . . . . . . . . . . . . . . . . . . 37

Chapter 3. Systen Operation . . . . . . . . . . . . . . . . . . . . . . . . . . . 42

3.1 Radiometer Performance . . . . . . . . . . . . . . . . . . . . . 42

3.1.1 Noise J'emperature . . . . . . . . . . . . . . . . . . . . . . . . 42

3.1.2 Calibration ............................ . 42

3.1.3 Operating Parameters . . . . . . . . . . . . . . . . . . . 43

3.1.4 System Testing . . . . . . . . . . . . . . . . . . . . . . . 43

3.1.4.1 Magnetic Dependence . . . . . . . . . . . . . . . . . . . 44 44

3.1.4.2 Radio Interference and Other Tests . . . . . . . . . . . . . . . 44

3.2 Data Collection ... . . . . . . . . . . . . . . . . . . . . 44

3.2.1 Flight Scheduling . . . . . . . . . . . . . . . . . . . . . 44

3.2 .2 July 1981 Flight . . . . . . . . . . . . . . . . . . . . . . . . . 45

3.2 .3 November 1981 Flight ........................ . 47

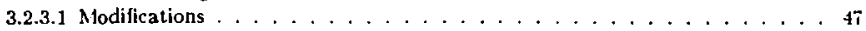

3.2.3.2 Flight . . . . . . . . . . . . . . . . . . . 18

3.2.4 April 1082 Flight . . . . . . . . . . . . . . . . . . . . 50

3.2 .4 Modifications . . . . . . . . . . . . . . . . . . . 50

3.2 .4 .2 Flight ........................... . . 52

3.2 .5 November 1082 Flight . . . . . . . . . . . . . . . . . . 53

3.2.5.1 Modifications . . . . . . . . . . . . . . . . . 55

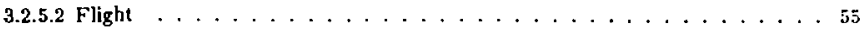

3.2.5.3 Post Mortem . . . . . . . . . . . . . . . . . . . 56

Chapter 4. Data Reduction and Processing . . . . . . . . . . . . . . . . . . . 59

4.1 Data Format . . . . . . . . . . . . . . . . . . . . . . . . 59

4.2 Beam Positional Reconstruction . . . . . . . . . . . . . . . . . . . G0

4.3 Data Editing and Sky Coverage . . . . . . . . . . . . . . . . . . . . . 61

4.4 Offset Analysis and Removal . . . . . . . . . . . . . . . . . . . . . 62

4.5 Conversion to Antenna Temperature and Correction to Barycenter . . . . . . . . . B6

Chapter 5. Data Analysis . . . . . . . . . . . . . . . . . . . . . . . 68

5.1 Spherical Harmonic Fits . . . . . . . . . . . . . . . . . . . . 68

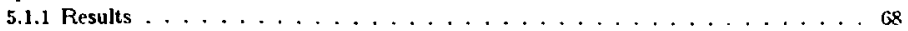

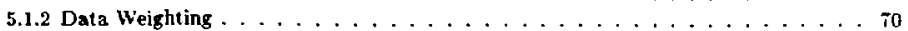

5.1.3 Chi-Square Statigtics and Hourly Averages . . . . . . . . . . . . il

5.1.4 Individual Flight Dipole Fits . . . . . . . . . . . . . . . . . . i6

5.1 .5 Galactic Effects and Models . . . . . . . . . . . . . . . . . . . . . . . . . . . . . . . . . . . . . . . . .

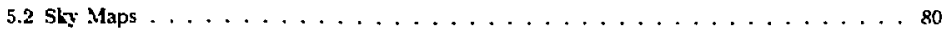


5.3 Systematic Error Analysis . . . . . . . . . . . . . . . . . . 82

5.3.J Errors of the First Kind . . . . . . . . . . . . . . . . . 84

5.3.1.1 Galactic Sources . . . . . . . . . . . . . . . . . . . 84

5.3.1.2 Interplanetary Sources . . . . . . . . . . . . . . . . 85

5.3.1.3 Ionospheric Free-Free Emission . . . . . . . . . . . . . . . 86

5.3.1.4 Almospheric Moleculas Emission . . . . . . . . . . . . . . . 86

5.3 .1 .5 Ground Pjckup . . . . . . . . . . . . . . . . . . 87

5.3.1.6 Balloon Contributions . . . . . . . . . . . . . . . 89

5.3.1.7 Sy nchronous System Modulations . . . . . . . . . . . . 89

5.3.2 Errors of the Second Kind . . . . . . . . . . . . . . . . . . . . 90

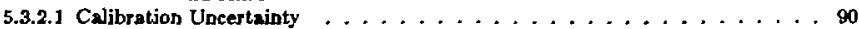

5.3.2.2 Pointing Errors . . . . . . . . . . . . . . . . . . . . 91

5.4 Summary and Conversion to Thermodynamic Temperature . . . . . . . . . . . . 91

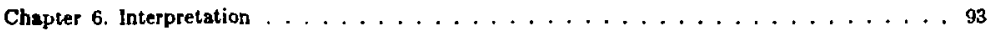

6.1 Dipole Anisotropy . . . . . . . . . . . . . . . . . . . . . . 93

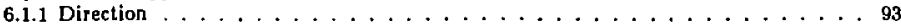

6.1 .2 Magnitude ............................ 95

6.1 .3 CBR Spectral Distortion . . . . . . . . . . . . . . . . . . 96

6.1.4 Solar Velocity . . . . . . . . . . . . . . . . . . . . . . 101

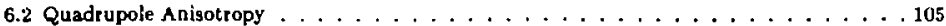

6.3 Galactic Emission . . . . . . . . . . . . . . . . . . . . 107

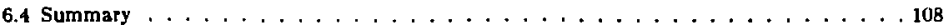

Appendix A. Relativistic Flux Trangformation . . . . . . . . . . . . . . 109

A.1 Phase Space Density . . . . . . . . . . . . . . . . . . . . . 109

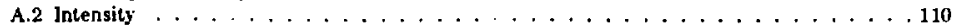

Appendix B. Radiometers . . . . . . . . . . . . . . . . . . . 112

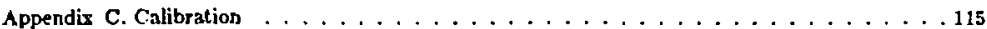

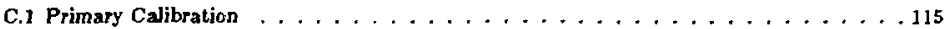

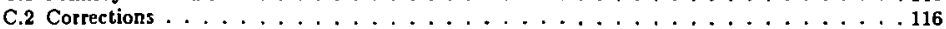

C.3 Stability of and Error in Calibration . . . . . . . . . . . . . . . . . 117

Appendix D. Linear Least Squares Estimation . . . . . . . . . . . . . . . . . 121

D.1 Best Fit Solution . . . . . . . . . . . . . . . . . . . . . 121

D.2 Uncertainty in Fitted Parameters . . . . . . . . . . . . . . . . . . . . . 122

D.3 Comparison of Independent Solutions . . . . . . . . . . . . . . . . . . 123

Appendix E. Generation of Sky Maps . . . . . . . . . . . . . . . . . . . . . . . . 125

E.1 Normal Equations . . . . . . . . . . . . . . . . . . . . . . . . 125

E.2 Gauss-Seidel Sparse Matrix Inversion . . . . . . . . . . . . . . . . . . . . . 127

E.3 Jacobi Iteratjve Method . . . . . . . . . . . . . . . . . . . . . . 128

E.4 Non-Iterative Method . . . . . . . . . . . . . . . . . . . . . . . . . . . . . 129

Appendix F. Radiometer Parts . . . . . . . . . . . . . . . . . . . . . . . . . . . . .

Appendix G. Azimuthal Anisotropy Coeftlcients . . . . . . . . . . . . . . . 131

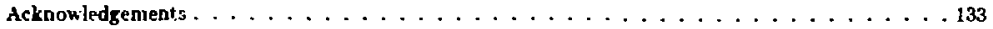

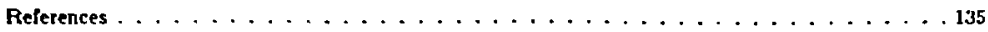




\section{Figures}

Page

Figure 1.1. Woody-Richards Spectrum $\ldots \ldots \ldots \ldots \ldots$

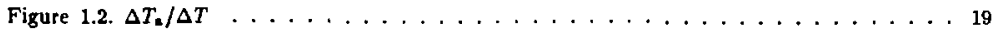

Figure 2.1. CBR Signal and Competing Backgrounds . . . . . . . . . . . 24

Figure 2.2. Calculated Zenith Atmospheric Errigsion at $30 \mathrm{~km} \ldots \ldots \ldots \ldots$

Figure 2.3. General Differential Radiometer Block Diagram . . . . . . . . . . . . . 28

Figure 2.4. Block Diagram of the $90 \mathrm{GHz}$ Anisotropy Radiometer . . . . . . . . . . . 30

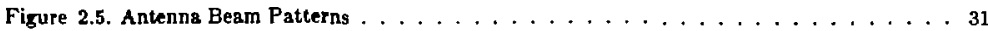

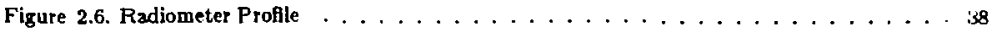

Figure 2.7. Perspective View of Gondola $\ldots \ldots \ldots \ldots \ldots \ldots \ldots$

Figure 2.8. Balloon and Flight Train $\ldots \ldots \ldots \ldots \ldots \ldots \ldots \ldots \ldots \ldots \ldots \ldots \ldots$

Figure 3.1. November 1981 and April 1982 Flight Paths . . . . . . . . . . . 49

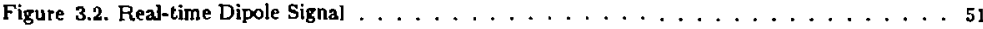

Figure 3.3. Northern Sky Coverage $\ldots \ldots \ldots \ldots \ldots \ldots \ldots \ldots$

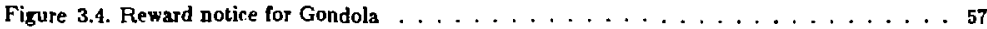

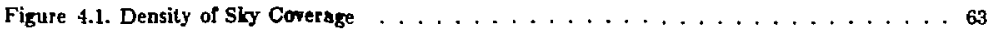

Figure 4.2. November 1981 Flight Data . . . . . . . . . . . . . . . . . . . 64

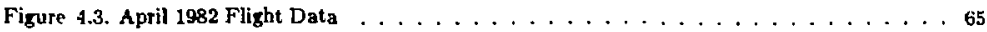

Figure 5.1. Hourb Sine and Cosine Anisotropy Coefficients . . . . . . . . . . i3

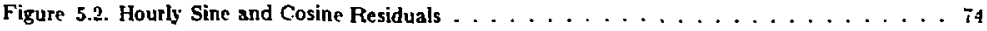

Figure 5.3. HI Emission in the Northern Sky . . . . . . . . . . . . . . ig

Figure 5.4. Reconstructed $90 \mathbf{G H z}$ Stymep $\ldots \ldots \ldots \ldots \ldots \ldots$

Figure 6.1. Consistency of $24.5 \mathrm{GHz}$ and $90 \mathrm{GHz}$ Dipole Anisotropies as a function of TCBR . . 99 


\section{Tables}

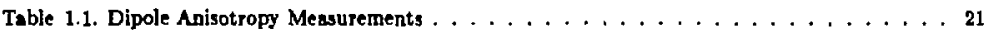

Table 5.1. Spherical Harmonic Basis Functions $\ldots \ldots \ldots \ldots$

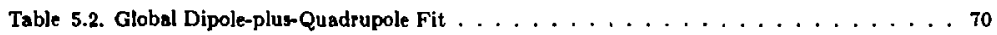

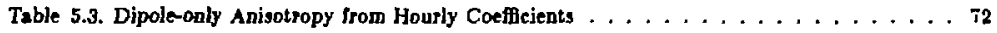

Table 5.4. Dipole-plus-Quadrupole Anisotropy from Hourly Coeffleients . . . . . . . . . . 6

Table 5.5. Dipole-only Fits . . . . . . . . . . . . . . . . 78

Table 5.6. Efrects of Galactic Cuts $\ldots \ldots \ldots \ldots \ldots \ldots$

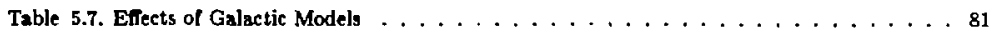

Table 5.8. Contributions to Total Error . . . . . . . . . . . . . . . . 92

Table 5.9. Dipole-plus-Quadrupole Anisotropy at $90 \mathrm{GHz} \ldots \ldots \ldots \ldots$. . . . . . . . 92

Table 6.1. $90 \mathrm{GHz}$ Dipole Anisolropy . . . . . . . . . . . . . . . 94

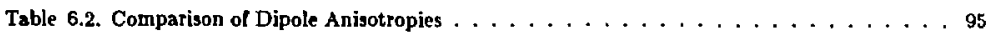

Table 6.3. Measured and Predieted Dipole Anisotropies . . . . . . . . . . . . . 98

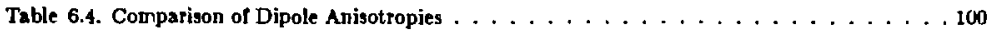

Table 6.5. Solas Peculiar Velocity . . . . . . . . . . . . . . . . . 103

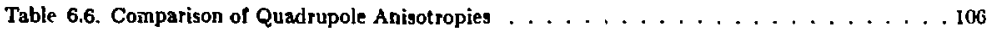

Table C.1. Pop-up Calibrator Ground Observations . . . . . . . . . . . . . 120 
Chopter 1

\section{Introduction and Theory}

People have speculated about the nature of their surroundings for as long as they hare been aware of them. Quantitative cosmology, requiring accurate observations as well as world models which motivate and provide interpretation for observations, is somewhet more recent.

The earliest un verse models were animistic - early man jmbued his surroundings with spirits and demons based upon his own motivations and emotions. Magical explanations gave way to mythological ones as civilizations developed, and systematic, if not physical, descriptions were given for the nature of the world and its origin. Subsequent efiorts to quantify and physically motivate descriptions of the universe represent what Harrison (1981) has called the transition from anthropocentric to anthropometric cosmologies. If not the center of the universe, man is at least a means by which the universe measures itself.

\subsection{A Pocket History of Cosmology}

\subsubsection{Early Earth-Centered Models}

Some of the earliest surviving attempts to relate astronomical observations to cosmological modeis, and to measure properties of the universe on scales larger than were immediately accessible, came from the aicient Greeks. By about the fourth centwry BCE, the Greel astronomers 
and philosophers had generally agreed on a universe model consisting of a spherical, stationary earth suspended at the center of a rotating sphere which carried the stars (Kuhn, 1957). This world view, although not unanimously adopted, was elaborated by Aristotle and others and became institutionalized in Christian theology by Thomas Aquinas and his thirteenth century contemporaries.

\subsubsection{The Copernican Revolutions}

In 1543 , Copernicus initiated a change in cosmological world view which removed the earth from its privileged location at the universal center. Successive displacements have expanded mankind's view of the universe and in many ways have further removed us from preferential status. Observations of non-uniformity in the distribution of stars led to the understanding that our sun was embedded within a larger system - the galaxy. In the late 1700's, Herschel placed the sun at the center of the Milky Way. By 1918, Shapley's observations of the anisotropic distribution of globular clusters showed the sun's actual position to be in the outskirts of our galaxy. The question of whether our "Island Universe" was unique or was rather one of many similar systems was resolved by Hubble's observations of the spira! nebulae, which showed them to be galaxies in their own right located far outside our own. Hubble's further discovery that the recession velocity observed for a galasy was proportional to its distance established the idea of an expanding universe.

Hubble's Law, that recession velocity equals a constant $\boldsymbol{H}_{0}$ times distance, does not mandate a privileged location for our galaxy. In a homogeneous, isotropicaliy expanding universe, oòservers at all locations will find the recession velocities of other galaxies to be proportional to their distances. For each observer, however, there will be a unique comoving frame of reference in which this proportionality between distance and velocity is independent of direction. The frame of reference defined by our galary, then, is special to the ertent that, this isotropy bolds. 


\subsection{Modern Mathematical Cosmology}

Modern cosmological models have taken, as their basis, what was stated by Einstein and developed by Milne (1933) as

The Cosmological Principle:

All places in the oniverse are equivi:ent.

This is merely the Coper:-an idea that we do not possess a privileged status or location, and that the universe at large scales is bomogeneous. Since we can only indirectly examine the distant universe, the Cosmological Principle must be postulated: it cannot be experimentally verifled. Some models had adopted the stronger postulate (Bondi and Gold, 1948) of

The Perfect Cosmological Prinr:ple:

All places and times in the universe are equivalent.

However, the steady-state models resulting from this assumption have not been able to explain the observed evidence of change in the universe. As one looks farther out in space (and therefore further back in time), the universe looks different, so the present is not equivalent to the past. The cosmic background radiation, discussed below and the main subject of this thesis, gives perhaps the clearest evidence of such erolution.

\subsubsection{Gezeral Relativity}

Einstein's General Theory of Relativity provides the mathematical framework in which to test and express models describing the nature of our universe on tL largest scales ar 1 earliest times. The theory relates how the geometry of space-time influences the mass distribution contained within it, and how the mass in torn determines the geometry. Within General Relativity, different assumptions ecncerning the mass distribution and overall structure of the 
universe can be codifled into diffesent cosmological models; the features and predictions of the different models can then be compared with observations.

\subsubsection{Big Bang Cosmologies}

The simplest cosmological models describe universes which are homogeneous and isotropic. All points are equivalent, and at any point all directions are equivalent. Robertson and Walker showed in 1935 and 1936 (Misner et al., 1973) that in all such universes, space-time can be separated into a curved three-dimensional space and a fourth time coordinate which can be consistently deflned by any comoving observer. The geometry of space is determined by a distance scale parameter $\boldsymbol{R}$ which is a function only of time, being independent of position. The expansion or contraction of the universe, represented by the time erolution of the scale parameter, is determined by the distribution of matter and energy.

The "hot big bang" cosmology, which today is the most widely accepted description of the history and large-scale structure of our nniverse, is built upon on a Robertson-Walker spacetime. It represeats a class of solutions to the Einstein equations derived by A. Friedmann in 1922 and G. Lemaitre in 1927 (Misner et al., 1973) in which the universe expands and cools from an initial state of extreme temperature and density. Hubble's Law is a necessary consequence of the expansion of the universe embodied in these models.

In its barest form, a big bang universe model begins in a singularity; its subsequent properties are parameterized by elapsed time as measured by comoving observers. A more convenient parameter for many purposes is the ratio of the distance scale parameter $R$ at a given epoch $t_{0}$ to its value at some other time $t$. Taking $t_{0}$ as the present time, this ratio can be shown to be equal to the ratio between the wavelength of a photon now, measured by a local comoving receiver, to its wavelength at time $t$, measured by a distant comoving observer: 


$$
\frac{\lambda\left(t_{0}\right)}{\lambda(t)} \equiv(1+z(t))=\frac{R\left(t_{0}\right)}{R(t)}
$$

The first identity defines the red shift parameter $z$.

Another important parameter in Robertson-Walker nniverses describes the matter density. In the simplest models, universes having less than critical density will always orpand following the initisl singularity; spz ze is open (inflite) and negatively curved. Universes hoving greater than the critical density will eventually stop expanding and will recontract into a "big crunch"; space in these universes is closed (flnite) and positively curved. Universes having exactly critical density will always expand, but at an ever decreasing rate; space is inflnite and flat. The ratio between the actual density of the universe and the critical density is denoted $\Omega$, so $\Omega=1$ is the value separating open from closed universes. Relativity theory, cosmology, and big bang models are described at various levels in many works (Misner et al., 1973; Weinberg, 1972; Weinberg, 1977; Silk, 1980, to give just a few).

\subsection{The Cosmic Background Radiation}

Whether or not big bang models can validly be extrapolated all the way back to a singularity at $t=0$, they have had great success in explaining many features of the universe today. The cosmic background radiation (CBR), a nearly isotropic, nearly blackbody radiation fleld giving the universe an effective temperature of about $3 \mathrm{~K}$, has a natural interpretation as highly red-shifted thermal radiation remaining from a much earlier epoch. When it was some nine orders of magnitude hotter, this radiation was responsible for photo-dissociating deuterium which would otherwise have formed in the early universe. As a result, the combination of protons and neutrons into more stable heavier nuclei was prevented. Deuterium could persist when the

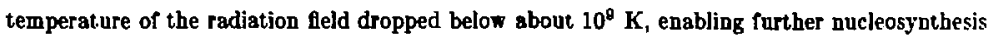
in which essentially all neutrons then present became bound into helium nuclei. The helium 
mass fraction of the universe today, thought to be between 0.20 and 0.25 (Y2.3g et al., 1979), is consistent with this scenario.

The radiation fleld continued to maintain thermal equiljbrium with matter until the universe expanded and cooled another sir orders of magnitude. At the epoch of recombination, when the temperature had dropped to about $4000 \mathrm{~K}$, the ionized plasma formed nentral hydrogen and helium and broke thermal contact with the radiation.

\subsubsection{CBR Prediction}

Several p8pers written in the late 1940's suggested that the universe msy have passed through a hot early stage in which heary elements were formed via thermonuclear reactions. Gamow (1946) argued the necessity of considering non-equilibrium nucleosynthetic processes in an expanding universe. Accounting for expansion time scales, Gamow (1948) showed that particle number densities of about $10^{18} \mathrm{~cm}^{-3}$ at temperatures of $10^{9} \mathrm{~K}$ would be required in order for an appreciable fraction of protons and neutrons to form heavier nuclei. These conditions represent a universe dominated by radiation. AJpher and Herman (1949) calculated that the present temperature of that radiation would be on the order of $5 \mathrm{~K}$.

At about the same time, experimental apparatus capable of measuring thermal radiation in the microwave band was being developed by Dicke at the MTT Radiation Laboratory (Dicke, 1946). In the process of making atmospheric absorption measurements, Dicke and colleagues put a limit of $20 \mathrm{~K}$ on the cosmic radiation temperature at three wavelengths near $1 \mathrm{~cm}$ (Dicke et al., 1946).

\subsubsection{Discovery}

Detection and identiflcation of the cosmic background radiation came some tro decades 
later. In 1965, Penzias and Wilson at Bell Laboratories reported finding an eflective zenith noise temperature $3.5 \pm 1.0 \mathrm{~K}$ higher than expected at $7.3 \mathrm{~cm}$ wavelength (Penzias and Wilson, 1965). The excess power was "isotropic, unpolsrized, and free from seasonal variation" to the limits of their observation.' A companion paper by Dicke, Peebles, Roll, and Wilkinson (1965) from Princeton University suggested a cosmological origin for this power as greatly red-shifted blackbody radiation which had been in thermal equilibrinm with ionized matter in the early universe.

The Princeton research had originated independently of the work at Bell Labs. Dicke had been considering oscillating universe models in which elements generated in one cycle would be destroyed by the extremely high temperatures occurring in the next contraction. By the summer of 1964, Dicke realized that red-shifted blackbody radiation remaining from a hot epoch, if present, should be observable. A literature seurch for existing upper limits to the temperature of a cosmological radiation fleld yielded the $20 \mathrm{~K}$ flgure Dicke himself had made in the 1940's, as well as a limit of $15 \mathrm{~K}$ set in 1961 by the total system temperature of the apparatus later used by Penzias and Wilson (Wilson, 1979). To extend these results, Roll and Wilkinson of the Princeton group construeted a radiometer to measure cosmic background radiation at 3.2 cm. This experiment was in progress when news of the Bell Labs discovery reached Princeton. Roll and Wilkinson's publication, within a year of the initial CBR discovery papers, reported a temperature of $3.0 \pm 0.5 \mathrm{~K}$ at over twice the frequency of the Penzias and Wilson observation and strongly supported the thermal origin of the radiation (Roll and Wilkinson, 1966 and 1967). The highly thermal and highly isotropic nature of the CBR, as conflrmed by these and subsequent. measurements, is generally taken as conflrmation of its primordial origin.

\footnotetext{
${ }^{1}$ The limits on isotropy and polarization in the orizinal detectlon of the CBR, not explicitly stated in Penzias and Wilson (1065), were $0.3 \mathrm{~K}$ and were independent of most of the errors contributing to the $1.0 \mathrm{~K}$ ucertainty in the CBR absolnte temperature (Wilson 1070).
} 


\subsubsection{Properties}

The CBR was quickly realized to be one of the few directly accessible probes of conditions and events in the early universe. Therefore, characterizing the CBR's properties as completely as possible became a high priority. Weiss (1980) has reviewed the status of CBR observations in the past twenty years.

\subsubsection{Spectrum}

Any distortion of the CBR spectrum from a purely thermal form would be of great interest since it might provide information about physical processes occurring in the early universe. In the low-frequency Rayleigh-Jeans region, the spectrum is consistent with a blackbody having a temperature (given by the weighted average of 21 measurements between 0.33 and $12 \mathrm{~cm}$ wavelength) of $2.76 \pm 0.07 \mathrm{~K}$ (Smoot et al., 1983; Weiss, 1980). At and above the peak, the measurement of Woody and Richards (1979) indicates a higher flux than the Rayleigh-Jeans measurements and shows deviation trom a thermal spectrum at the two standard deviation level. The Woody-Richards spectrum has excess flux near the peak and a deficiency above when compared to the $2.96 \mathrm{~K}$ blackbody spectrum having the same integrated flux. Figure 1.1 shows the Woody-Richards spectrum along with some lower frequency measurements and a $2.96 \mathrm{~K}$ blackbody curve.

An indirect, but potentially quite precise, measurement of the CBR intensity at speciflc frequencies near and above the peak is obtained from incerstellar cyanogen observations. The relative strengths of two lines in the $\mathrm{CN}$ absorption band at $3874 \hat{\mathrm{A}}$ - one from the ground state and the otber from the first rotationally excited state - permit determination of the relative populations of the states and hence of the $\mathrm{CN}$ excitation temperature. Since excitation mechanisms other than the CBR are possible, the excitation temperature is an upper bound to 


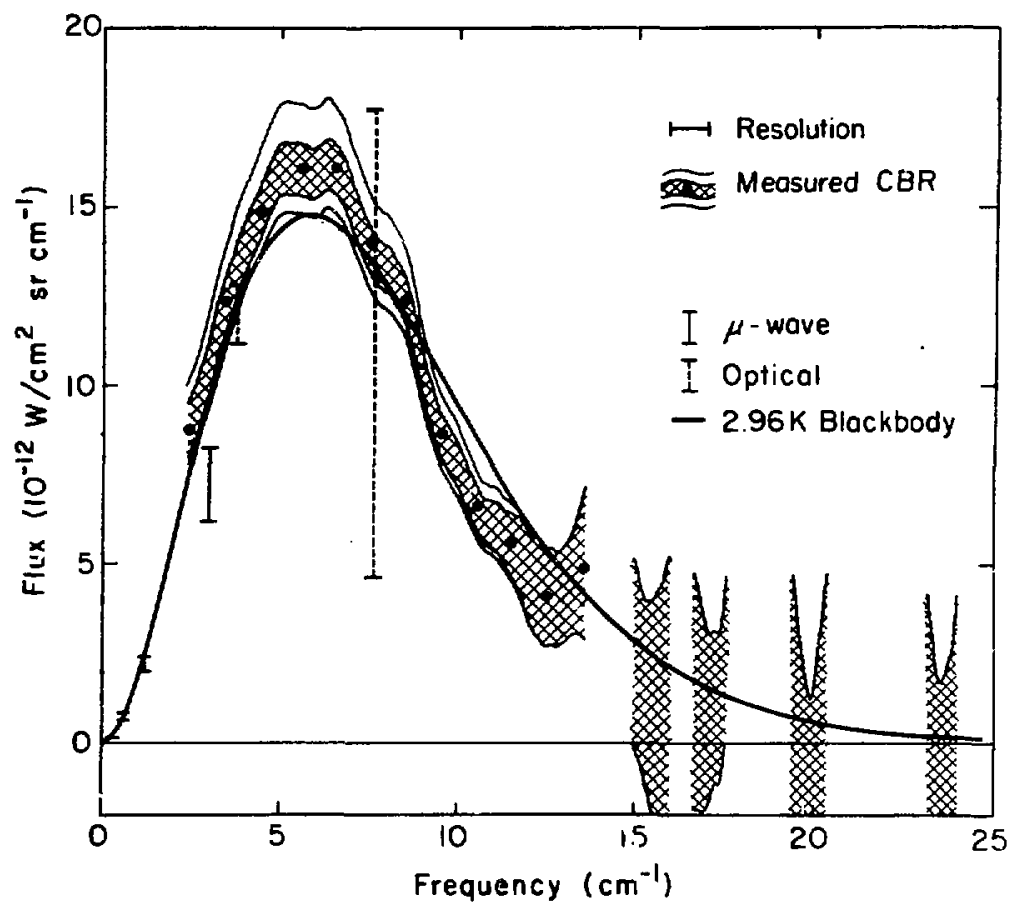

Figure 1.1. Woody-Richards spectrum (Woody and Richards, 1979). 
the CBR temperat ure at the frequency of the transition linking the cyanogen states. Subsequent to the Woody-Richards measurement, Meyer and Jura (1984) have made a high signal-to-noise measurement of this excitation and flnd $T_{\mathrm{CBR}} \leq T_{\mathrm{ex}}=2.73 \pm$ i.j.: $\tilde{\mathrm{n}}$ at $1 \mathrm{i} \cdot \mathrm{i} \mathrm{GE} z$, in disagreement. with the Woody-Richards results.

\subsubsection{Polarization}

Any anisotropy intrinsic to the CBR which was established prior to the CBR's last scattering will be accompanied by a net linear polarization due to the polarization dependence of Thompson scattering (Rees 1968). Lubin, Melese, and Smoot (1983b) have placed a 95\% confldence level limit of $0.2 \mathrm{mK}$ on any linearly polarized component at $3 \mathrm{~mm}$ wavelength.

\subsubsection{Isotropy}

The present universe is quite transparent to microwave radiation, so the CBR we measure today has propagated freely from regions of the universe where the line-of-sight optical depth becomes significant. Its angular intensity distribution carries information about the structure of the uigiverse on these scales. A dipole anisotropy of a part in one thousand of the CBR, attributable at leas". in part to our motion relative to a comoving frame, is by now well establisbed (Smaot et al., 1977; Gorenstein, 1978; Corey, 1978; Cheng et al. 1979). Further investigation of angular anisotropy in the CBR forms the subject of this work.

\subsection{Isotropy of the CBR}

\subsubsection{Isotropy of the Universe}

There are many mechanisms which can gencrate anisotropy in the cosmic background radiation. Indeed, the greater challenge for theoretical cosmology is explaining the CBR's unifor- 
mity. In standard big bang models, which treat the early universe as a radiation-dominated Roberson-Vialker space-time, radiation reaching us from opposite parts of the sky comes from regions which have never been in causal contact. ${ }^{2}$ Without postulating specialized initial conditions, it is hard to understand why these independent regions should be at the same temperature.

\subsubsection{Chaotic Cosnology}

Misner (1968) hoped to account for the present isotropy by showing that in a large class of homogeneous but anisotropic models, initial anisotropy would damp out due to dissipative processes such as neutrino viscosity. However, it was later shown that the temperature of the cosmic background radiation itself limits the amount of dissipation that could have occurred in the very early universe (Barrow and Matzner, 1977). Collins and Hawking (1973a), eramining the same question of whether initially homogeneous but anisotropic universes evolve towards isotropy, showed that those which do form a set of measure zero in the space of all spatially homogeneous models. In sther words, a given chautic universe model will in general not become isotropic. Instead, Collins and Hawking proposed an alternate explanation for the observed isotropy of our universe: only universes expanding just fast enough to avoid recollapse, those being the ones which approach isotropy, can frrm galaxies containing observers who measure isotropy.

\subsubsection{Anthropic Principle}

This last argument is an invocation of what Carter (1974) termed

The Anthropic Principle:

What we can expect to observe must be restricted by the conditions necessary for our existence as observers.

\footnotetext{
${ }^{2}$ Taking the sorface of last scattering to be at a red shit of 1500 . retions more than $3 \sqrt{\Omega}$ angalar degrees apart on that surface are cavsally disconnected (Weinberg, 1972, p. 525).
} 
Carter sees this as a qualification of the Copernican principle discussed earlier. We do not... occupy a central location in the uriverse, but the fact that we exist indicates that our situation is necessarily privileged to some extent.

Many authors have used anthropic arguments to explain what otherwise might need to be attributed to coincidence or to undiscovered physics. Consider two very large but apparently independent dimensionless numbers: the ratio of the electrical to gravitational forces between proton and electron, and the Eubble time, or age of the universe, measured in the length of time light takes to cross a classical electron radius. Both are on the order of $10^{40}$. Dirac's Large Number Hypothesis explains their near-equality by postulating a physical relationship which sets them equal. Dicke (1961), on the other hand, reasons that our very presence requires the existence of heavy elements. We cannot measure a universe to be younger than the lifetime of the stars required to produce those elements, which is on the order of several billion years. However, if the universe were much older than that, all stars and all observers would have died out. Therefore, we measure its age to be $10^{40}$ "light-electrons" old because we are bere to measure it.

Carr and Rees (1979) discu' a number of relationships between mass, length, and time scales in the universe. Many of these relationships have straigbtforward physical derivations; others can be "explained" only by involing anthropic arguments. Carr and Rees find the latter approach somewtat unsatisfying, from a physical point of view, for three reasons: the anthropic explanations supply post-boc rationalizations rather than predictions, they may embody overly anthropomorphic assumptions coneerning what is necessary for the existence of observers, and they cannot provide exact values for the constants that they are able to relate by order of magnitude. Nevertheless, Carr and Fiees feel that the antbropic explanation was the only candidate (apart from the less general Large Number Hypothesis) able to account for the "remarkable coincidences" displayed by nature. 


\subsubsection{The Inflationary Universe}

At least one of these "remarkable coincidences" of cosmology, the isotropy of the CBR, may have found a more physical justification in recent theoretica! work. Isotropy is a puzzle in standard models since, as has been mentioned, different areas on the surface of last scattering are causally disconnected; no signal traveling from any common origin can have standardized all of them. However, the size of the region to which a given point can be causally connected, its particle horizon, depends on the rate of expansion of the universe.

If we lived in a static oniverse which had a fnite age $t$, our present particle horizon would have a proper radius of $c t$. Light signals which left points on our horizon at $t=0$ would now just be arriving after having traversed the distance $c t$ between the horizon and us. In an expanding universe, the relationship between time and horizon size is more complicated. Our particle horizon at the present time still represents the portion of space which we are aware of - that portion from which we have received light signals. But the present location of the points on that horizon depends on how the universe expands during the time that the light is traveling to us. Furthermore, the size of the largest region which could have been causally connected at some earlier time, which is the particle horizon size for an observer at that time, depends on the nature of the expansion.

The scale parameter in early Friedmann-Robertson-Walker universes (standard big bang models) grows as $t^{\frac{1}{2}}$, for a radiation-dominated universe, or $t^{\frac{2}{3}}$, for a matter-dominated one. This is not fast enough to permit all points on the surface of last scattering of the cosmic background radiation to be inside each other's particle horizons at the time the CBR was last scattered. But if the scale parameter were to have grown exponentially rapidly for a period in the early universe, a very large region could be causally connected today. Extrapolating the present slower expansion rate back to the big bang, we greatly underestimate the size of the region which act ually would have been in contact during and before the exponential growth. 
Inflationary universe models (Guth, 1981; Press, 1981) isotropize the universe by splicing just such a period of exponential growth into the early nniverse. To see how this period arises, we must examine the concept of the cosmological constant. In the early twentieth century, when Einstein formulated the General Theory of Relativity, the universe was believed to be static. On discovering that only dynamic solutions existed to his origina! feld equations, Einstein modifled the equations by adding an additional term. This added cosmological constant represents a longrange repulsive force which just balances the gravitational attraction of mass over long distances, preventing collapse of the universe. It does so at the expense of destroying the equivalence of General Relativity to classical Newtonian gravitational theory in the limit of weak gravitational flelds. After Hubble verifled that the universe actually was expanding, Einstein abandoned the modiffeation, later calling it "the biggest blunder of my life." (Misner et ad, 1972, p. 758).

The cosmological constant can be interpreted as a "vacuum" energy density attributable to free space, differing from ordinary matter or energy ("substance") density in that it bas a negative effective pressure. The solution to the fleld equations for a universe having a cosmological constant, but devoid of matter, is exponential expansion. If there is a cosmological constant today, it must be sufficiently small so that its equivalent vacuum energy density is negligible compared to the mass-energy density of any system adequately described by Newtonian gravitation. Existing limits are sufficient to determine that the present, dynamics of the universe are not dominated by a cosmological constant term.

However, according to certain particle physics grand unifled gauge theories (GUTs), a large cosmological constant could have temporarily arisen in the very early universe. In these models, all non-gravitational interactions are equivalent at extremely high temperatures. When the universe cools below a critical transition temperature within a few orders of magnitude of $10^{28} \mathrm{~K}\left(10^{15} \mathrm{Gev}\right)$, it undergoes a phase transition in which the strong interactions between quarks become distinguishable from the electroweak interactions among leptons. The symmetric 
phase in whict all interactions are equivalent, which is the minimum energy state at higher temperatures, becomes a "alse vacunm" state which has a highar potential energy than the "true vacuum" state of broken symmetry. In the inflationary GUT models, the form of the vacuom potential is such that the universe becomes supercooled - it takes a relatively long time to evolve from the false vacuum to the true racuum state. During that evolution, the potential energy of the false vacuum acts as a cosmological constant, and the universe andergoes a period of exponential expansion. As the aniverse reaches the true asymmetric vacu am state, the false vacuum energy is dissipated as heat, ending the exponential growth. The re-heated universe subsequently evolves according to the standard big bang models.

Inflationary scenarioj are extremely attractive mechinisms for explaining the present isotropy of the universe. They also can explain some other presently outstanding cosmological puzzles. Recall that the difference between the dimensionless density $\Omega$ and unity is a measure of the curvature of space. In a spatially flat universe, $\Omega=1 . \Omega$ is our universe at present is most likely bounded by the limits 0.01 and 10 (Weinberg, 1972, p. 475 181; Misner et af., 1972, p. 796-797); recent studies of galactic dynamics indicate a value on the order of 0.2 (Davis and Peebles, 1983a). Since $\Omega$ is on the order of one, space is ciose to flat. But the deviation between $\boldsymbol{\Omega}$ and unity increases with time in big bang universes. Therefore, having $\Omega$ within two orders of magnitude of unity at present requires its value to have been within a part in $10^{15}$ of unity when the universe had a temperature of $10^{10} \mathrm{~K}$ - - thus, the flatness problem (Guth, 1981).

The exponential growth phase of inflationary scenarios solves the flatness problem. During that phase, the cosmological constant (representing racuum, rather than substance, energy density) dominates the dynamics of the universe; the expansion rate then is exactly the rate for which the racuum energy density is the critical density. Space in an exponentially growing universe is flat. During the phase transition ending the exponential growth period, vacuum energy emerges as substance (radiation or particles) which remains at the critical -lensity. Space is flat. 
before and after the transistion, and it remains flat in the period of power-law expansion which follows. Inflationary models predict that the total density of the universe today should equal the critical degsity to very high accuracy.

Inflationary universe models, then, provide, answers for some of the presently ontstanding problems with conventional big bang models. They predict that the universe at present is critically dense, and more relerant to this thesis, that there should be no detectable intrinsic anisotropy in the CRR attributable to large-scale inhomogeneity in the early universe. The models are the subject of intense scru'iny by both particle physicists and cosmologists (Barrow and Turner, 1982).

\subsubsection{Non-Intrinsic Sources of Anisotropy of the CBR}

\subsubsection{Peculiar Velocity and Antenna Temperature}

At some levei, we certainly expect so measure anisotropy in the CBR. Motion of the earth about the sun, the sun about the galaxy, and the galaxy relative to a comoving frame fill introduce anisotropy into measurements of the CBR even if the CBR is isotropic in a comoving frame. Motion through a blackbody radiation field will preserve its blackbody spectrum but will give it an angle-dependent temperature (Peebles and Wilkinson, i 968 ). If $\Theta$ is the angle between the direction of observation and the direction of motion, the observed temperature is

$$
T(\theta)=T \frac{\left(1-\beta^{2}\right)^{2}}{(1-\beta \cos \theta)}
$$

where $T$ is the temperature in the isotropic frame and $\theta=\frac{v}{6}$ is the dimensionless velocity of the moving observer relative to the isotropic frame. For sinall values of $\beta$, the expression can be linearized to yield

$$
T(\theta)=T(1+\beta \cos \theta) .
$$


representing a ftrst-order or dipole anisotropy with a fractional magnitnde equal to $\beta$. in a blackbody radiation fleld of $2.7 \mathrm{~K}$, a velocity of $330 \mathrm{~km} / \mathrm{sec}$ induces a thermodynamic ejpole anisotropy of $3 \mathrm{mK}$.

In general, consider the trassformation of a radiation field described by intensity I (power per unit area per unit solid angle per unit bandwidth). At frequency $\nu_{0}$ teflne the intengity spectral index $\alpha$ to be the $\operatorname{logarithmic}$ derivative $(\theta \log I / \theta \log \nu)$ at $\nu_{0}$. If $I$ is isotropic in soms reference frame, the linearized intunsity snisotropy : $:$ a frame moving at velocity $g$ is gival i seo Appendix $\dot{i}$ ) by

$$
\begin{aligned}
I^{\prime} & =I_{0}^{\prime}(1+\beta(3-\alpha) \cos \theta) \\
\dot{\Delta} I^{\prime} & =:_{0}^{\prime} \beta(3-\alpha)
\end{aligned}
$$

where $\Delta I^{\prime}$ is the amplitude of the dipolf: anisotropy. Note th th the spectral indes as well as the velocity enter into the fors.ula. In priticular, an isotropic radiation field having an intensity proportional to $\nu^{3}(\alpha=3)$ is isotropic in all frames: the frequency dependence $\left(\sim \nu^{3}\right)$ exactly balances the contraction of solid angle $\left(\sim \nu^{2}\right)$ and the trassformation $c /$ pheton energy $(\sim \nu)$ induced by the observer's motion.

A microwave radiometer measures power received in one polarization with a flxed collecting area, solid angle scceptance, and handwidth. It is therefore directly measuring intensity I. Let the antenna terperature corresponding to intensity $I$ measured at frequency $\nu$ be defined as

$$
T_{a}=\frac{c^{2}}{2 k \nu^{2}} I
$$

If measured in the Rayleigh-Jeans portion of the spectrum, the antenna temperature of a blackbody flling the beam of a microwave radiometer eqrals the blackbody's thermodynamic temperat.ure. In the Wien limit, antenna temperature drops exponentially with frecuency. 
Letting $x$ be the parameter $h \nu / k T$, the antenna temperature and thermodynamic temperature are related by

$$
\begin{aligned}
T_{a} & =\frac{x}{e^{x}-1} T, \text { and } \\
\Delta T_{a} & =\frac{x^{2} e^{x}}{\left(e^{x}-1\right)^{2}} \Delta T .
\end{aligned}
$$

In prineiple, the frequency dependence of a motion-induced antenns tempersture anisotropy permits the spectrum of a radiation feld to be determined. Figure 1.2 shows bow a CBR thermodynamic temperature anisotropy $\Delta T$ appears as a frequency-dependent antenna temperature anisotropy $\Delta T_{a}$ whose form is a function of the CBR spectrum. Plotted is the ratio $\Delta T_{a}$ to $\Delta T$ for both $2.7 \mathrm{~K}$ and $3.0 \mathrm{~K} \mathrm{CBR}$ spectra.

\subsubsection{Gravitational Radiation}

An additional source of non-intrinsic anisotropy in the CBR would be the quadrupolar and smaller-scale distortion produced by long-wavelength gravitational radiation (Burke, 1975).

\subsubsection{Lutrinsic Anisotropy}

\subsubsection{Anisotropic Cosmologies}

Large-seale anisotropy in the stracture of the universe will produce red shifts dependent on direction. Bawking (1969) and Collins and Bawking (1973b) discuss the forms taken by the CBR due to rotation or vorticity, shear, and peculiar velocities in spatially homogeneous but anisotropic cosmological models. The CBR appearance due to a particular anisotropy of the universe is strongly dependent on the type of anisotropic model studied. For example, shear introduces a quadrupolar variation in the CBR in two particular anisotropic models; in other models the variation is more complez. In addition, the magnitude of induced anisotropy depends on how long ago the radiation was last scattered. 
ANTENNA TEMPERATURE ANISOTROPY

F :EQUENCY DEPENDENCE

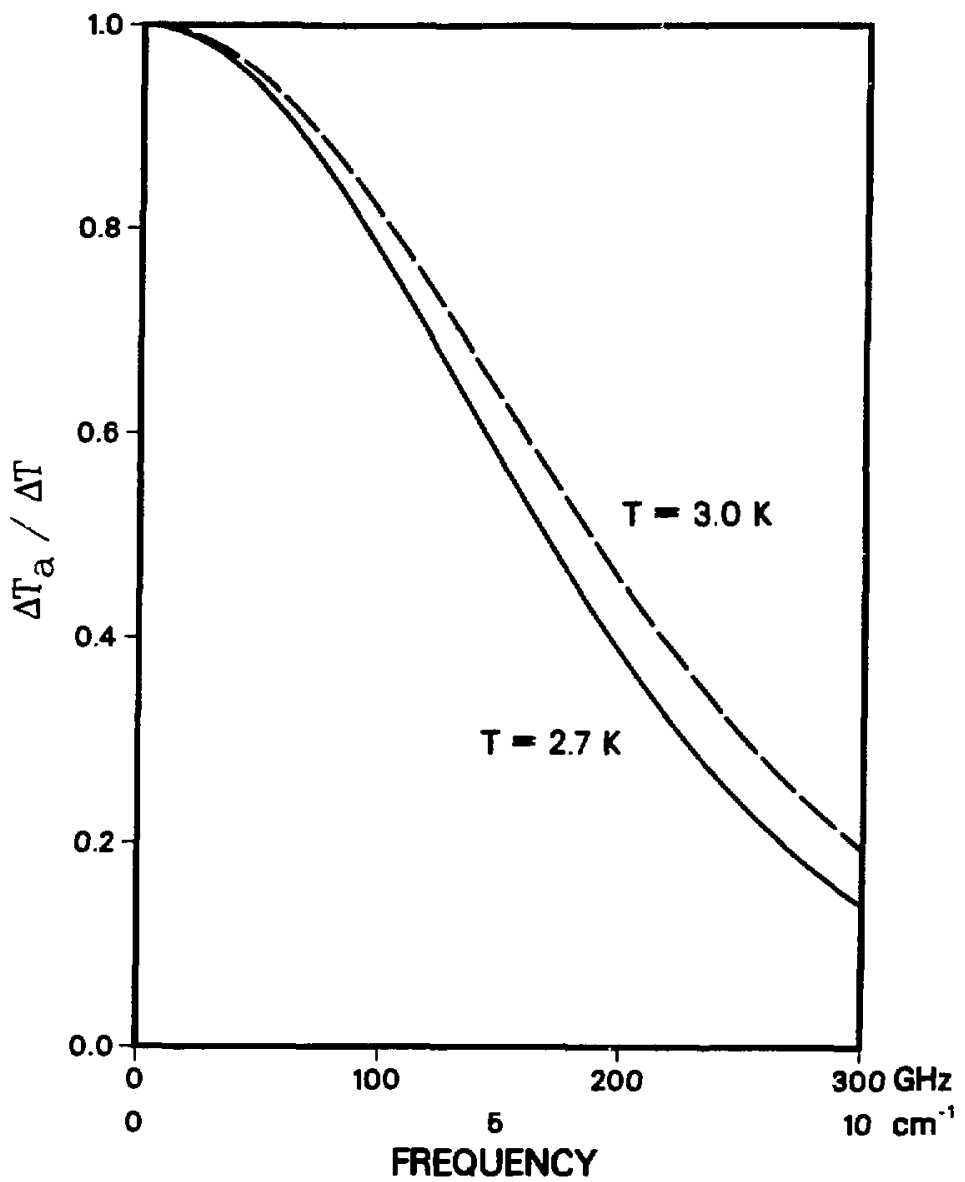

Figure 1.2. $\Delta T_{a} / \Delta T$ for $2.7 \mathrm{~K}$ and $3.0 \mathrm{~K}$ blackbody $\mathrm{CBR}$ spectra. 


\subsubsection{Inhomogeneous Matter Distribution}

Matter inhomogeneity certainly exists today. Therefore, perturbations must have existed at some level in the early universe as well. They could have influenced the CBR in three ways - intensity (temperature) fluctuations on the surface of last scattering, differential red shifts due to velocity fluctuations of the last scatterers, and differential gravitational red shifts imparted by matter inhomogeneities between the surface of last scattering and the observer. Sachs and Wolfe (1967) used the last mechanism, along with the then-existing $1 \%$ upper limit on CBR anisotropy, to bound large-scale density fluctuations in the universe on scales of 1000 megaparsecs to be under $10 \%$. Since ther, considerable work has been done predicting what anisotropy in the CBR would be expected given various density distributions and cosmological models (e.g. Silk and Wilson, 1981; Peebles, 1982; Fabbri et al., 1982; Hogan et al., 1982).

\subsubsection{Previous Experimental Results}

Searches for large-scale CBR anisotropy have been undertaken since the discovery of the CBR in 1965 . These are differential observations which directly compare power from different parts of the sky. Systematic environmental and instrumental effects are minimized by observing the same sky regions with difierent experimental conflgurations and differencing the measurements so that only CBR anisotropy contributes to the result. Results of previous measurements are given in Table 1.1. Only the most recent publication in ongoing series of experiments have been listed. The gap between 1971 and 1979 represents work which has been incorporated into the post-1979 publications.

\subsubsection{Ground-Based Obserrations}

The first anisotropy measurements were done at microwave trequencies (centimeter 
Table 1.1. Dipole anisotropy measurements.

\begin{tabular}{|c|c|c|c|c|c|c|}
\hline Source & Platform & $\begin{array}{c}\text { Altitude } \\
\text { (lm) }\end{array}$ & Type $^{t}$ & $\begin{array}{c}\nu \\
(\mathrm{GHz})\end{array}$ & $\begin{array}{c}\lambda \\
(\mathbf{c m})\end{array}$ & $\begin{array}{c}\text { Dipole Magnitude } \\
\text { (mK) }\end{array}$ \\
\hline $\begin{array}{l}\text { Wilson and Penzias, } \\
196 \pi\end{array}$ & Ground & 0 & $\overline{\mathbf{R}}$ & 4.1 & 7.32 & $<100$ \\
\hline $\begin{array}{l}\text { Partridge and Wilkinson, } \\
1967\end{array}$ & Ground & 0 & $\overline{\mathbf{R}}$ & 9.4 & 3.19 & $0.9 \pm 2.1$ \\
\hline Conklin, 1969 & Mountain & 3.8 & $\overline{\mathbf{R}}$ & 8.0 & 3.75 & $1.6 \pm 0.8$ \\
\hline Boughn et al., 1971 & Ground & 0 & $\overline{\mathbf{R}}$ & 35.0 & 0.86 & $7.5 \pm 11.6$ \\
\hline $\begin{array}{l}\text { Smoot and Lubin, } \\
1979\end{array}$ & Aircraft & 20 & $\overline{\mathbf{R}}$ & 33.0 & 0.91 & $3.1 \pm 0.4$ \\
\hline Fabbri et al., 1980 & Balloon & 40 & B & $100-600$ & $0.05-0.30$ & $2.9 \pm 1.3$ \\
\hline $\begin{array}{l}\text { Muehlner and Weiss, } \\
1980\end{array}$ & Balloon & 39 & $\bar{B}$ & $90-300$ & $0.10 \cdot 0.33$ & $2.8 \pm 0.8$ \\
\hline Boughn et al, 1981 & Balloon & 27 & $\overline{\mathbf{R}}$ & $\begin{array}{l}19.0 \\
24.8 \\
31.4 \\
46.0\end{array}$ & $\begin{array}{l}1.58 \\
1.21 \\
0.95 \\
0.65\end{array}$ & $\begin{array}{l}3.0 \pm 0.8 \\
4.0 \pm 0.3 \\
3.7 \pm 0.3 \\
3.9 \pm 1.0\end{array}$ \\
\hline Firsen et al., 1983 & Balloon & 25 & $\bar{R}$ & 24.5 & 1.21 & $3.18 \pm 0.17$ \\
\hline This work, 1983 & Balloon & 28 & $\overline{\mathrm{F}}$ & 90.0 & 0.33 & $3.48 \pm 0.23$ \\
\hline
\end{tabular}

${ }^{1} \mathbf{R}=$ Radiometer; $\mathbf{B}=$ Bolometer

2 Thermodynamic Temperature for $T_{C B R}=2.7 \mathrm{~K}$

wavelengt hs) from the ground. Except for Conklin (1969), the ground-based observations provided upper limits. Conklin reported the first signiflcant non-zero result for the equatorial 24-hour (dipole) anisotropy projected to 32 degrees declination. His result agrees with later observations but involved extrapolating a large galactic correction from lower frequency observations with a poorly known spectral index.

\subsubsection{Aerial Observations}

Deflnitive observations of CBR dipole anisotropy have all been made from the air. Radiometric observations have been done by the Princeton group from balloon-borne platforms (Corey, 1978; Cheng et al., 1978; Boughn et al., 1981; Fizsen et al., 1983) and by the Berkeley 
group from both the U-2 aircraft and balloons (Smoot et ah, 1978; Gorenstein, 1978; Lubin et af., 1983a) Far-infrared bolometric observations have also been made from balloon-borne platforms (Weiss, 1980; Fabbji et al., 1980) All aerial observations have measured the dipole anisotropy to have magnitude about $3 \mathrm{mK}$ in roughly the same direction, although there are statistically signiflcant discrepancies in magnitude and direction between experiments.

Radiometric anisotropy magnitudes in Table 1.1 have been converted from antenna temperature anisotropy to thermodynamic anisotropy according to equation $1.6 \mathrm{~b}$ by assuming the CBR to have a thermal spectrum with a temperature of $2.7 \mathrm{~K}$. Below $35 \mathrm{GHz}$, the ratio $\Delta T_{a} / \Delta T$ is insensitive to the exact lemperature or precise spectral shape of the CBR (Figure 1.2). At $90 \mathrm{GHz}$, the conversion factor of 0.81 becomes 0.85 if the CBR temperature is taken to be $3.0 \mathrm{~K}$ instead of $2.7 \mathrm{~K}$. Conversion of the wideband bolometric infrared measurements to thermodynamic temperature is highly dependent on the detector frequency response and the assumed spectrum of the CBR; the assumptions made for the infrared works quoted here were not explicitly stated by the authors.

Fabbri et al. (1980) reported detection of a "quadrupole-like" anisotropy with magnitude $0.9+0.4 \mathrm{mK}$. They were unable to characterize it in terms of second-order spherical harmonics due to insuffcient sky coverage. Reanalysis of the data (Ceccarelli et al., 1982) determined quadrupcle coefifients and attributed at least part of the quadrupole to an extended infrared source; however, the reanalysis was still unable to specify the dipole and quadrupole anisotropies completely. Boughn et al., (1981) reported significant quadrupolar anisotropy coefficients of $0.31 \pm 0.15 \mathrm{mK}$ and $0.54 \pm 0.14 \mathrm{mK}$ in the two second-order spherical harmonic components having twelve-hour periods in right ascension. This quadrupole detection was retracted in Fixsen et al. (1983), published simultaneously with the initial publication (Lubin et al., 1983a) of the work reported here. The experiment reported in this thesis does not support claims of quadrupolar anisotropy in the cosmic background radiation. 


\section{Chapter 2 \\ This Experiment}

\subsection{Design Considerations and Goals}

The two principal goals of this experiment were a desire to measure the background radiation anisotropy with high sensitivity and to do so at a frequency signiflcantly different from previous measurements. These two considerations, sensitivity and frequency, are not independent. The technology available to perform this experiment depends upon the operating wavelength, and the degree of sensitivity needed or desirable depends on what signal level is expected and what the competing backgrounds will be. The signal and the backgrounds are both frequency dependent.

\subsubsection{Choice of Frequency}

The most fundamental contaminant of measurements of the large-scale anisotropy of the cosmic background radiation is diffuse galactic radiation. Estimates of the galactic contribution to a seven-degree full width at half maximum (FWHM) bean on the galactic plane are identifled in Figure 2.1. Since the galactic contributions have different frequency dependences from the CBR and from each other, comparison of measurements at different frequencies can in principle 
SIGNAL AND GALACTIC BACKGROUND FREQUENCY DEPENDENCE

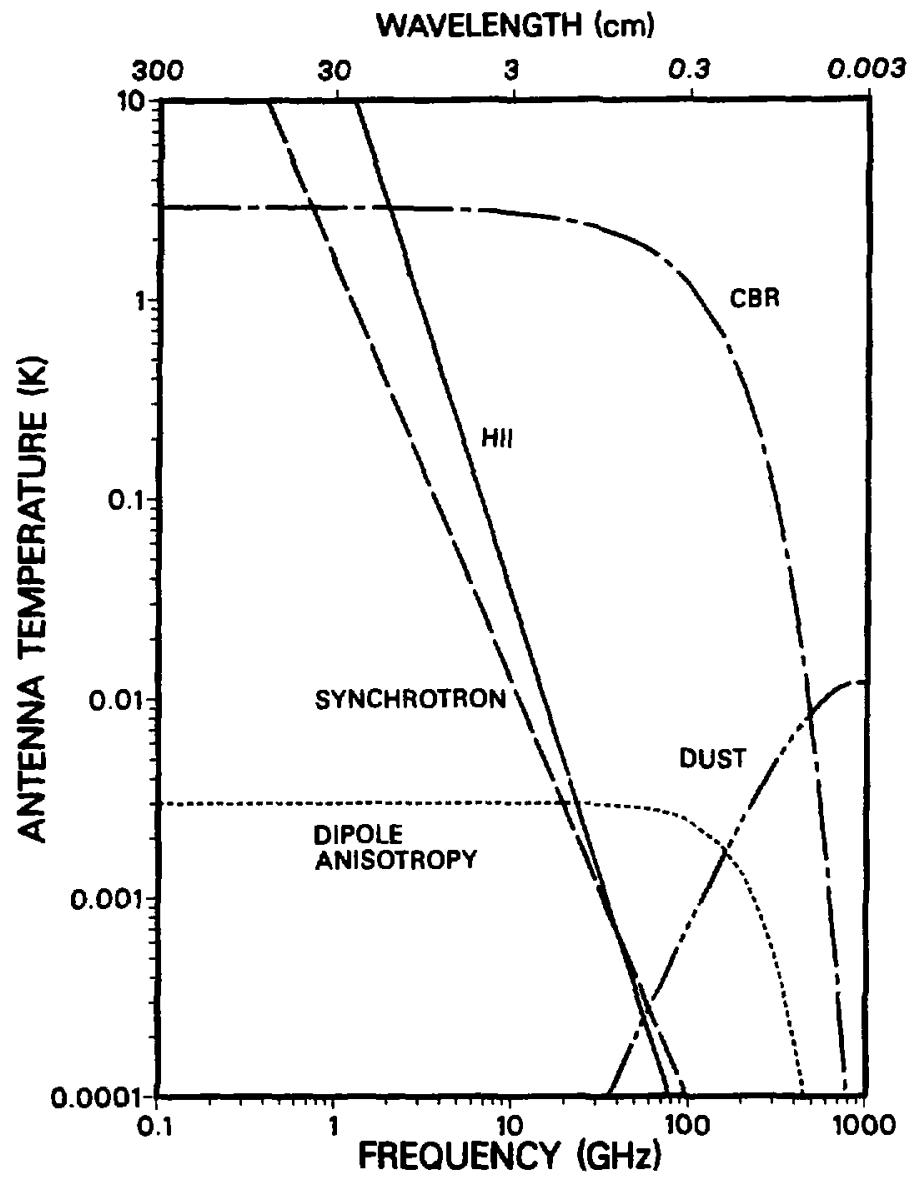

Figure 2.1. CBR signal and competing backgronnds. Galactic backgrounds are for a seven-degree beam on the galactic equator. 
decouple the various contributions. This procedure is of course limited by the accuracy to which the frequency dependences are known.

Synchrotron radiation from electrons in the galactic magnetic fleld, and thermal brehmsstrahlung from ionized hydrogen regions, are the dominant contributiors at centimeter wavelengths. In the infrared, thermal emission from interstellar dust predominates. If galactic radiation is signiflcant, its angular distribution must be understood before attributing anisotropy to the CBR. Preferably, anisotropy measurements should be done at a frequency where the galactic contribution is minimized. Figure 2.1 indicates a window near $90 \mathrm{GHz}$ where the total galactic emission is low. Exactly where this minimum occurs depends on how the dust emission is distributed spatially and spectrally.

Having a galactic window is of little use if one cannot see through the atmosphere. Figure 2.2 shows the calculated atmospheric zenith emission at $30 \mathrm{~km}$ altitude in the microwave region, indicating a window between two oxygen absorption peaks at 60 and $120 \mathrm{GHz}$. Within this window, the band between 86 and $92 \mathrm{GHz}$ is protected for radio astronomy and was selected for this experiment.

\subsubsection{Choice of Technology}

Microwave technology is continually improving, both in sensitivity and in accessible frequencies. Development of high-performance millimetes and sub-millimetes jadiometers has blurred the boundary between microwave and infrared. In the region where the two overlap, at a wavelength of about a millimeter, the distinctions are primarily in terminology and technique.

As to terminology, this microwave experimentalist will usually say the frequency of a 360 micro-electron-volt CBR photon is $90 \mathrm{GHz}$, whereas his infrared colleagues will label it $3 \mathrm{~cm}^{-1}$. Diflerences in technique are more tundamental. $A$ microwave receiver is a coherent detector - 


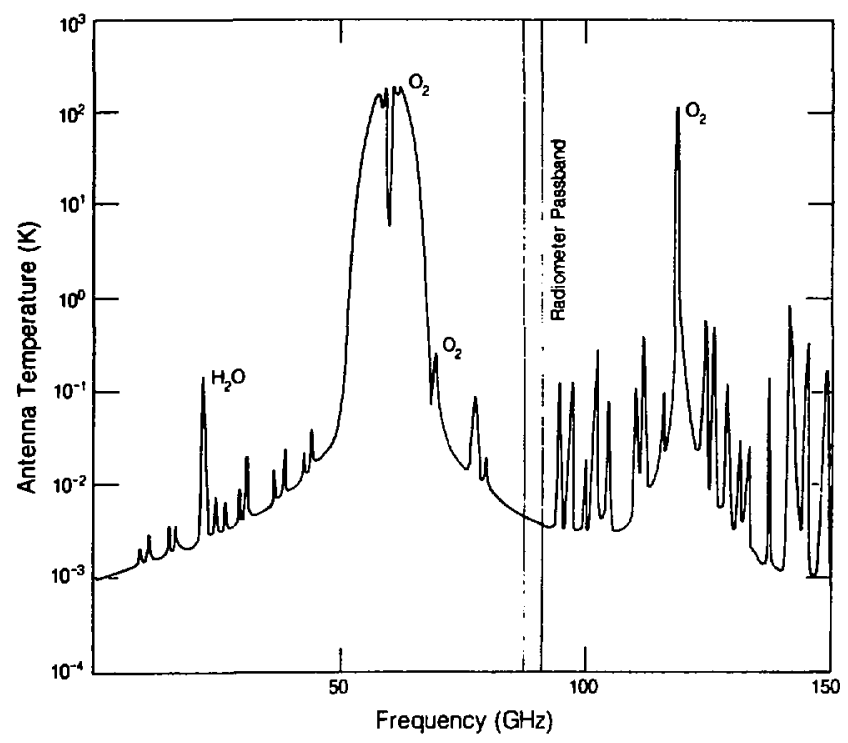

Figure 2.2. Calculated zenith atmospheric emission at $30 \mathrm{~km}$. All unlabeled peaks are ozone lines.

it can preserve the phase of the incident signal. A bolometer used to detect infrared radiation is an incoherent device sensitive only to total incident power. In isotropy measurements, where ultimately only the power is measured, both types of devices can he used. This experiment uses a cryogenically-cooled radiometer, which can be made more sensitive than a bolometer in the region near $90 \mathrm{GHz}$. More importantly, a radiometer's passband can be defined much more precisely than can that of a bolometer, making it possible to take betier advantage of windows between atmospheric emission peaks. The signal to noise ratio of a radiometer increases with the square root of the bandwidth of the detected radiation, whereas a bolometer's sensitivity is 
directly proportional to its bandwidth. Therefore, the bolometer is better able to utilize large bandwidths.

\subsubsection{Choice of Platform}

The differential radiometers nsed to measure anisotropy compare incident intensity from two sky patches located at equal zenith angles. Atmospheric emission, to first order, contributes equally to both. However, atmospheric variation and misalignment of the apparatus will canse the actual atmospheric contributions to differ. By using a high-altitude platform, total atmospheric emission (and therefore differential atmospheric emission) is reduced to acceptable levels. At $30 \mathrm{~km}$, the zenith atmospheric antenna temperature at $90 \mathrm{GHz}$ is $4 \mathrm{mK}$.

The required altitudes can be reached by balloon, rocket, or satellite. Rocket flights do not last long enough to make sufficiently sensitive measurements. An orbiting platform is ideal; flight duration, sky coverage, and elimination of atmospheric background make a satellite experiment particularly attractive for measuring the CBR anisotropy. The Cosmic Background Explorer (Mather and Kelsall, 1980) has been under NASA development since 1974 to do so, but it will not be launched before 1987 . This experiment was therefore designed to be carried by a sciericiflc balloon.

\subsection{Radiometer Description}

The heart of this experiment is a cryogenicallf-cooled differential radiometer (Appendix B) which uses the sky for both source and reference. Such a device, with an output proportional to the difference in intensity between areas in the sky, permits anisotropy to be measured directly without the need to make absolute measurements to the $10^{-4}$ level. Figure 2.3 shows a general block diagram of a differential radiometer used in this configuration. Having the two radiometer 


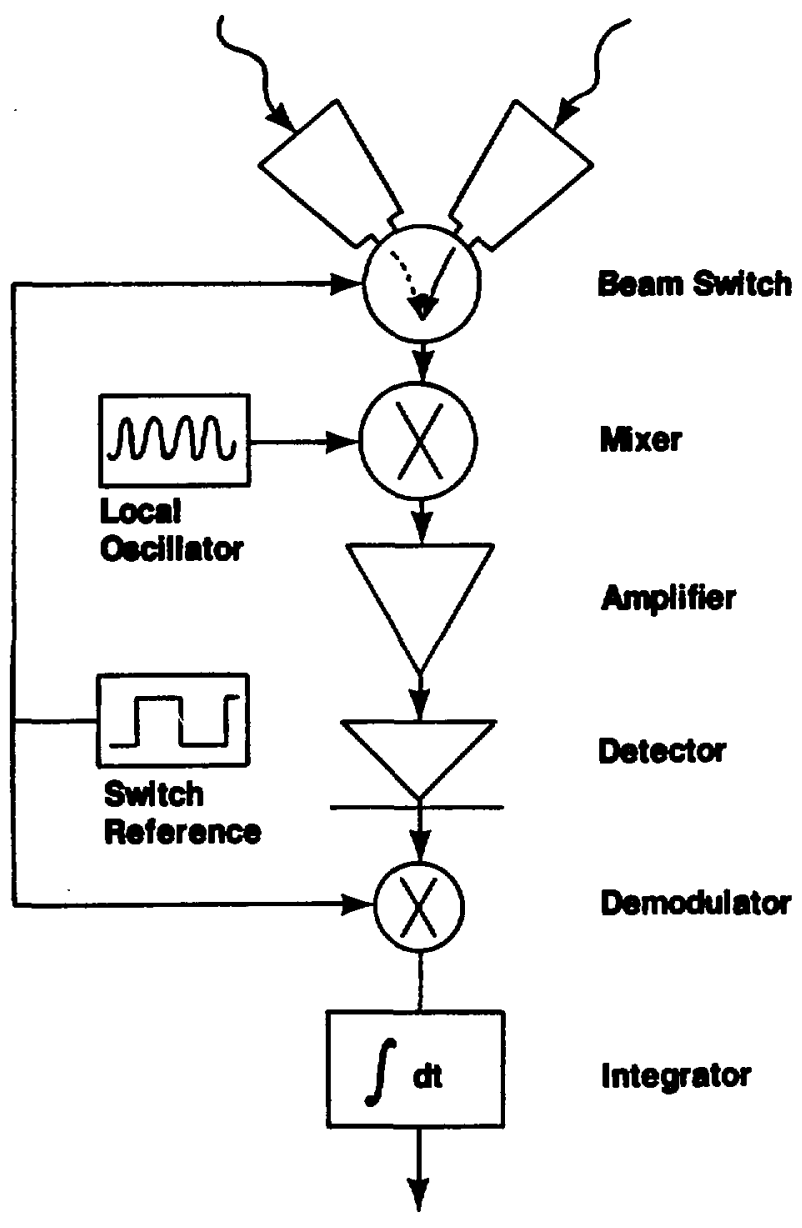

Ortput

Figure \&.S. General differential radiometer block diagram. 
felds of view at equal zenith angles balances residual atmospheric emission. Rotating the receiver, besides providing sky coverage, modulates anisotropy in the background radiation to distinguish it from asymmetry in the apparatus. Anisotropy will yield a difference signal which changes sign when the sky patches observed by each beam are interchanged; an asymmetry or offset will remain constant to the extent that the apparatus itself is unaffected by rotation. It is therefore very important to ensure that nothing internal to the receiver changes synchronously with rotation.

\subsubsection{Radiometer Bardware}

Since electronic waveguide switches at $90 \mathrm{GHz}$ have bigh loss and would significantly degrade the performance of a cryogenic system, the radiometer in this experiment switches between flelds of view using a rotating chopper wheel. The antenna, pointed towards the chopper at an angle of 45 degrees from vertical, alternately accepts reflected and transmitted radiation from two locations 90 degrees apart in the sky. A block diagram of this particular radiometer is given in Figure 2.4 .

\subsubsection{Cryogenic Temperature Components}

Horn and Coupler: Power passes into the cryogenic dewar ${ }^{1}$ through a 5 -mil thick mylar window and is collected by a corrugated conical horn cooled to $77 \mathrm{~K}$. The antenna half-power beam width is $\tau$ degir $^{-}:$, and the gain pattern for an equivalent horn scaled to $33 \mathrm{GHz}$ and measured at $31.4 \mathrm{GHz}$ (Janssen et al., 1979) is shown in Figure 2.5. Beam patterns of a $90 \mathrm{GHz}$ horn identical to the one used in this experiment bave been measured and are essentially the same as those sbown in Figure 2.5 (C. Witebsky. private communication). A circular-to-linear polarization transition and a $5.4 \mathrm{~cm}$ copper-plated section of thin-wall stainless steel wareguide

\footnotetext{
${ }^{1}$ Specific components used in the radiometer are itemized in Appendis $F$.
} 


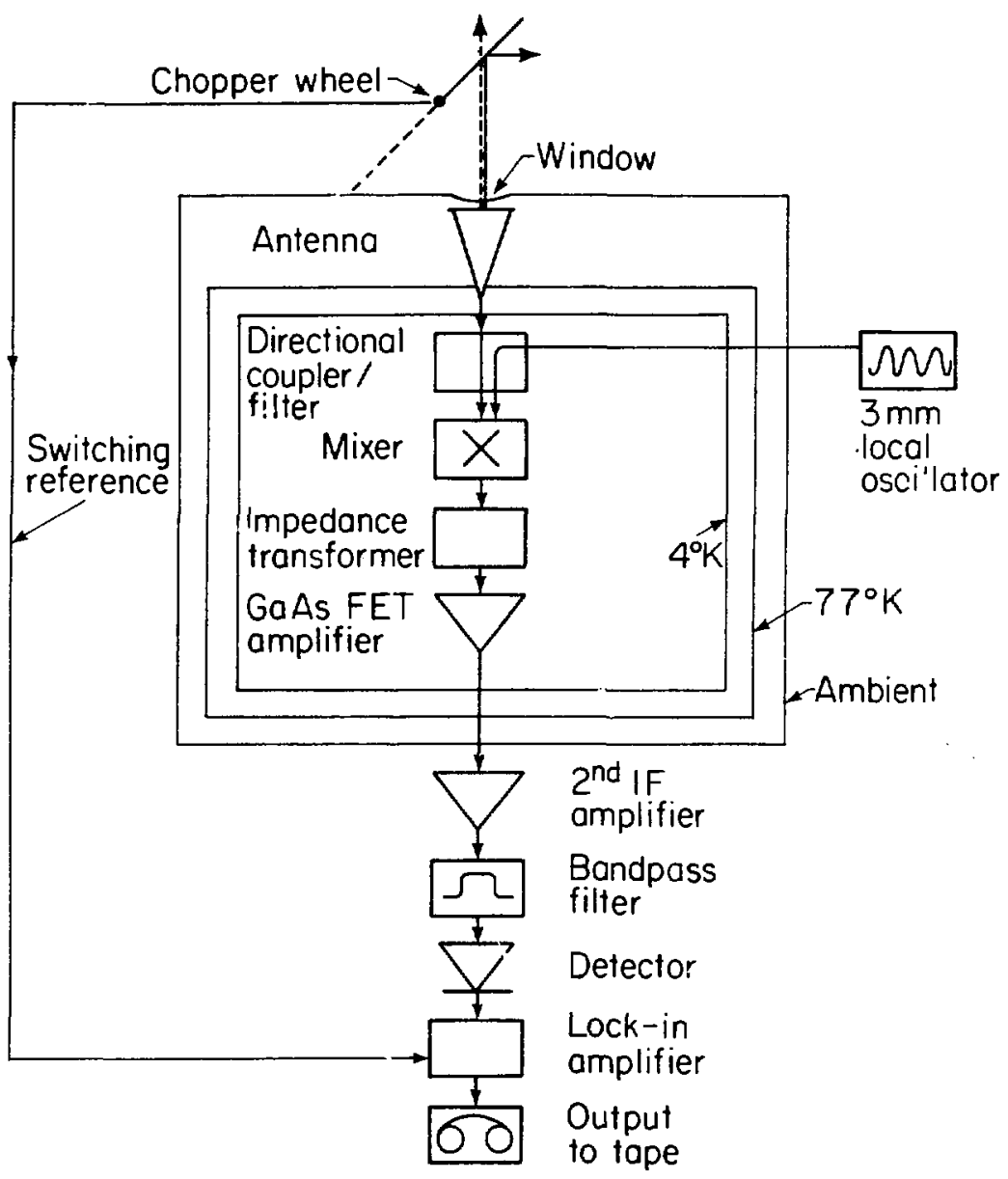

Figure 2.4 . Block diagram of the $90 \mathrm{GHz}$ anisotropy experiment. 


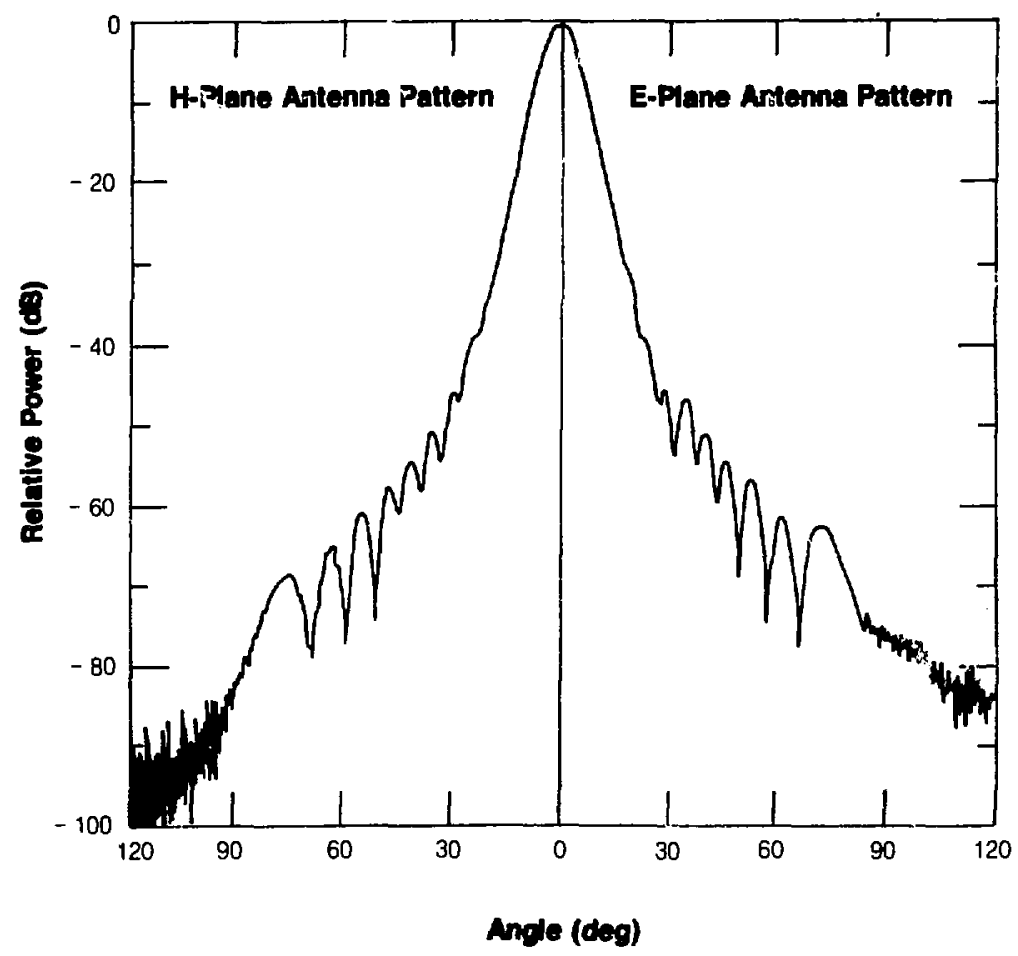

Figure 2.5. Antenna heam patterns. Data shown are for a proportionally scaled horn at $31.4 \mathrm{GHz}$ (Janssen et al, 1979); the $90 \mathrm{GHz}$ beam patterns are essentially identical. 
connect the horn to the components on the $4 \mathrm{~K}$ cold plate. Loss in the window and in the signal waveguide is less than $0.3 \mathrm{db}$.

The sigmal and the $90 \mathrm{GHz}$ local oscillator (LO) power fed separately into the dewar enter two ports of a tunable resonant ring diplezer (Davis, 1977). The mirer block is mounted on the third port and the fourth port is terminated. Insertion loss of the coupler for the signal is $0.3 \mathrm{db}$ at room temperature; local oscillator-to-mixer loss in the coupler when the coupler is toned fcr ma: imum LO throughput is $4 \mathrm{db}$ for the LO carrier and $23 \mathrm{db}$ for non-resonant LO sideband noise.

Mixer: From the coupler, LO and signal power pass through a step-reduced-height wavegnide transformer to a low-doped Schottky barrier diode. Noise theory of Schottky barrier mixers is discussed in Barber (1967). Weinreb and Kerr (1973) analyze cryogenically-cooled centimeter and millimeter mixers; the mixer used in this experjment is an updated version of the one described by Kerr (1975) and Cong, Kerr, and Mattauch (1979). The GaAs mixer diode is operated with a DC bias current of $780 \mu a m p$. It is mounted on a quartz microstrip and protrudes into the quarter-height WR-10 waveguide. A phosphor-bronze whisker extending across the waveguide contacts the metal surface of the Schottky barrier. Intermediate frequency (IF) power passes from the diode through a microstrip radio frequency choke and of the mixer block.

Behind the chip, an adjustable backshort tunes away the reactive component of the impedance seen by the junction. The tuning vernier on the ring coupler and the backshort are not accessible when the system is cooled. They were adjusted when the system was warm to maximize LO power coupied into the diode, as indicated by the change in diode voltage at constant bias current when the LO was turned on. When the system is cooled, the bias voltage is not as strongly affected by the $L O$.

IF Amplifer: A low-noise IF amplifer immediately following the mixer is essential to utilize the cooled mixer's low noise figure. The noise contributed by components downstream of the 
first IF amplifier is reduced by a factor equal to the first IF's gain and is therefore not signifcant. This receiver uses a cryogenically-cooled two-stage GaAs FET amplifer described by Williams and Lum (1980) and built in the University of California's Radio Astronomy Laboratory. The amplifier is optimized for performance at L-band frequencies between one and two GHz. The actual IF bandwidth of 1080 to $1670 \mathrm{MHz}$ was established by evaluating the receiver spot noise figure and gain as a function of frequency and maximizing the overall sensitivity. At $77 \mathrm{~K}$, the amplifler has a noise temperature of $20 \mathrm{~K}$ and a gain of $25 \mathrm{db}$ over an effective gain-weighted bandwidth of $540 \mathrm{MHz}$. Its output is attenuated $3 \mathrm{db}$ by the low thermal conductivity stainlesssteel rigid coaxial cable through which power leaves the dewar cold-space.

The amplifier functions best when viewing a source impedance of 50 to 70 ohms (Williams and Lum, 1980), whereas the incremental impedance of the biased mixer diode when cooled is closer to 200 ohms (Cong, Kerr, and Mattauch, 1979). Therefore, a broadband, partially tunable impedance transformer is used to match the two. While connected to the mixer, the transformer was trimmed to minimize VSWR over the IF amplifler operating band. The transformer also provides a terminal for feeding DC bias to the mixer.

\subsubsection{Non-Cryogenic Temperature Components}

Local Oscillator: A Gunn diode followed by a waveguide attenuator produces $6 \mathrm{mw}$ of $90 \mathrm{GHz}$ LO power which is further attenuated $10 \mathrm{db}$ by passage through a mylar window, an unplated stainless-steel waveguide inside the dewar, and the ring coupler. Somew bat less than one milliwatt is delivered to the mixer. The $L O$ power was adjusted to maximize radiometer sensitivity, which is only weakly dependent on the LO power level.

Second IF amplifier, Filters, and Detector: Outside the dewar, the radiometer signal passes through an isolator (which was later removed: see Chapter 3 ) and is amplified another $50 \mathrm{db}$ by the second IF amplifier. The $180 \mathrm{~K}$ noise temperature of this amplifier adds less than $2 \mathrm{~K}$ to the 
system noise, since its contribution is reduced by a factor equal to the first IF amplifier's gain. The IF passband is determined by an eight-pole bandpass filter following the second IF ampliffer. The signal then passes through a notch filter at the balloon telemetry transmitter frequency of 1485.5 $\mathrm{MHz}$ (see Chapter 3) and is attennated to keep the Schottky barrier detector diode in its linear range. After detection, the signal is fed to a lockin amplifier.

Beam Chopper and Lockin Reference: The beam is switched between sly patches with a polished aluminum rotating chopper wheel. The chopper emissivity into the mode accepted by the radiometer (linear polarization with $\mathbf{H}$ fleld vertical) can be calculated by considering the corresponding case of radiation incident on a conductor at 45 degrees with $\mathbf{B}$ fleld in the plane of incidence. The fractional power absorbed is given by

$$
\epsilon=\frac{2 \delta \cos 45^{\circ}}{\lambda}
$$

with $\delta$ the skin depth and $\lambda$ the free space wavelength over $2 \pi$. Upon substituting typical values for aluminum, $\epsilon$ equals $6 \times 10^{-4}$ at $90 \mathrm{GHz}$. Fractional absorption is equal to emissivity, so this value predicts emission on the order of $135 \mathrm{mK}$ from an aluminum chopper at $225 \mathrm{~K}$. Emissivity increases as the square root of resistivity, so a warmer chopper has a higher emissivity as well as a higher physical temperature.

The chopper wheel, having low emissivity and surrounded by turbulent air, is assumed to be at the ambient air temperature. However, the ambient temperature is difleult to measurt since the residual air at altitude does not make good thermal contact with sensors. The blackbody pop-up calibrator target, whose temperature ranged between 205 and $225 \mathrm{~K}$ during two different flights, most likely radiatively cooled below the ambient air temperature. The $225 \mathrm{~K}$ air temperature used in the emissivity calculation is that given in the U.S. Standard At mosphere (Cole et al., 1965; reprinted by Ulaby et al., 1981) for ambient temperature between 28 and 30 $\mathrm{km}$. The actual offset observed during flight is about $200 \mathrm{mK}$; the discrepancy can be attributed 
to the large uncertainty in the conductivity of aluminum alloys depending on composition and preparation and to the uncertainty in chopper wheel temperature.

The chopper is a tro-tobed wheel driven at 700 RPM and phase-locked to a crystalcontrolled oscillator. The beam switching trequency is $23 \frac{2}{3} \mathrm{~Hz}$. When the chopper is phaselocked, the oscillator provides the 23 f Hz reference signal to the lockin and also establishes the integration period of 50 switch-cycles (just over two seconds). The motor and clock circuits are electricaliy isolated from the rest of the apparatus.

Lackin Amplifter and Data Logger: The first stage of the synchronous-filter lockin amplifies the detector output. The amplified signal is used to charge one capacitor when the radiometer observes the direct beam and another when it observes the reflected beam. $A$ differential amplifier produces an output voltage proportional to the difference between capacitor voltages. During the $20 \%$ of each switch cycle when the edge of the chopper crosses the horn, the loekin inpat signal is blanked to eliminate spurious diffracted signals. As a result, one second of observation time corresponds to a net integration time of 0.8 second. The lockin output is "boxcar-integrated" for a period of 2.11 seconds, digitized, and recorded by an onboard tape logger.

\subsubsection{Support Equipment.}

\subsubsection{Magnetometers}

To reconstruct the positions of the direct and reflected beams, the radioneter's orientation is first determined relative to the earth's magnetic field. Two sets of magnetometers are included in the experiment. The primary system is a three-axis fuxgate unit; thr:e orthogonalymounted Hall probes provides a backup system. 


\subsubsection{In-Flight Calibrator}

To determine the stability of the radiometer calibration in flight, a small blackbody target made of iron-loaded epoxy was periodically rajsed into the benm. The target subtends about $1 \%$ of the beam solid angle and contributes an antenna temperature on the order of $1 \%$ of its physical temperature. At altitude, the calibrator showed that the system gain is stable to better than $\pm 1 \%$ over a flight. Calibration is discussed further in Appendix C.

\subsubsection{Power}

The system requires thirty watts of power with ap to thirty more watts going to heaters in the temperature regulators. This power was supplied by five pounds of lithium batieries on the radiometer's first flight, when weight was particularly critical, and by 100 pounds of rechargeable lead-acid gelled electrolyte cells on subsequent flights. All power supply voltages were regulated by non-switching series-pass DC regulators.

\subsubsection{Temperature Regulation}

Cryogenic temperature stability is maintained independent of altitude and ambient pressure by venting the liquid helium and liquid nitrogen tanks through absolute-pressure regulators. Temperatures inside the dewar are monitored at three locations with silicon diodes. One sensor on the horn measures the temperature of the liquid-nitrogen-cooled shield, and two on the $4 \mathrm{~K}$ cold plate measure the GaAs FET $\mathrm{F}$ amplifier and the mixer block lemperatures, respectively.

Temperatures of the non-cryogenic electronics were regulated with proportional heaters. Integrated circuit sensors on the chopper motor, the LO, the second IF amplifier, the support 
electronics cage, the dewar exterior, and the in-flight calibrator were used tc measure and record these temperatures; the heater currents were also recorded.

\subsection{System Configuration}

\subsubsection{Experiment and Gondola}

The radiometer has flown four times in four different conflgurations. The radiometer dewar, chopper wheel, and motor remained unchanged for the four flights and are shown in profile in Figure 2.6.

The dewar is tilted back with the horn viewing the chopper at a 45 degree angle. In this position, the dewar could hold about two liters each of liquid nitrogen and liquid helium, sufficient to keep the system cold for at least 18 hours. Surrounding the aperture for the horn is a hexagonal groundshield, shown in cross-section in Figure 2.6, which was designed to keep ground radiation from contributing more than $0.1 \mathrm{mK}$. This groundshield is supplemented by shielding on the gondola. The chopper is enclosed, except where it crosses the horn, by an electrically-conductive guard (not shown in Figure 2.6) which minimizes modulation of any stray radio power and prevents the loss of significant digits (i.e. the author's).

The electronics not mounted in or on the dewar, snd the flight batteries, are carried separately on the balloon gondola. Also on board is the telemetry and telecommand system linking the experiment to the balloon launching facility. The entire gondola is suspended under a motor and bearing which rotates the package. A perspective riew of the gondola as flown in April 1982 is shown in Figure 2.7.

2.3.2 Balloon and Flight Train

The required size of a scientiflc balloon depends on the weight of the apparatus and 


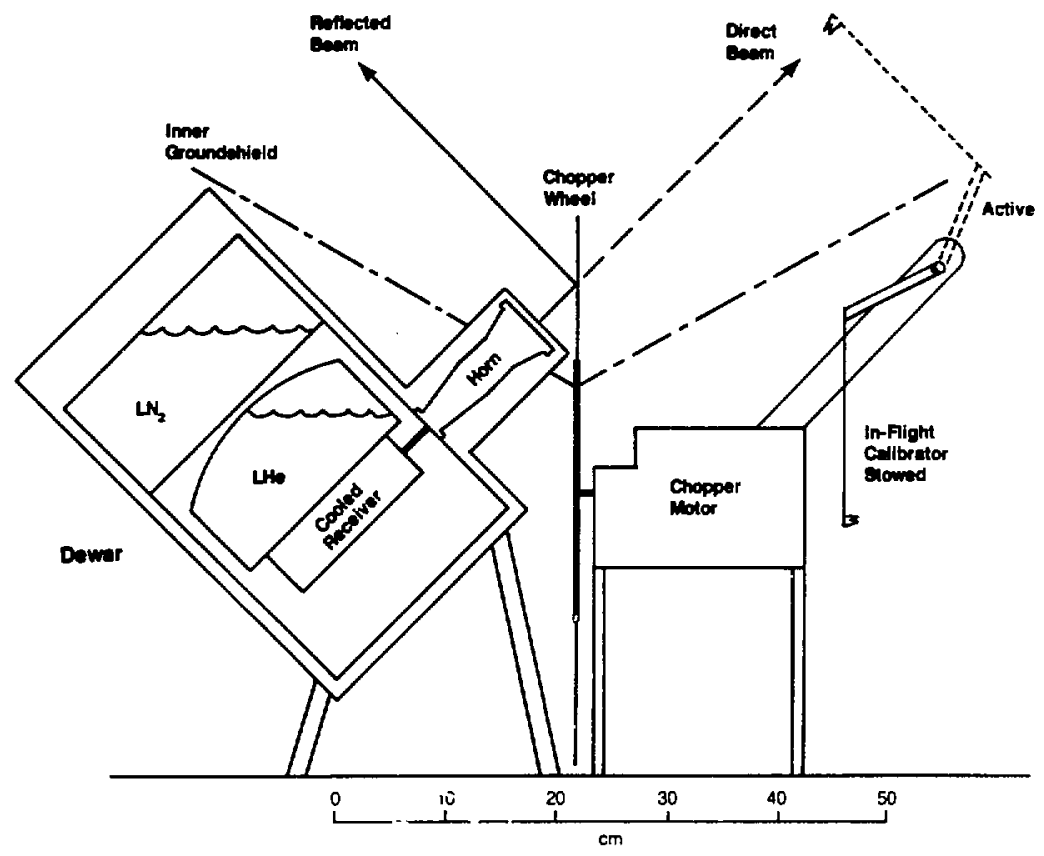

Figure 2.6. Radiometer profile. 


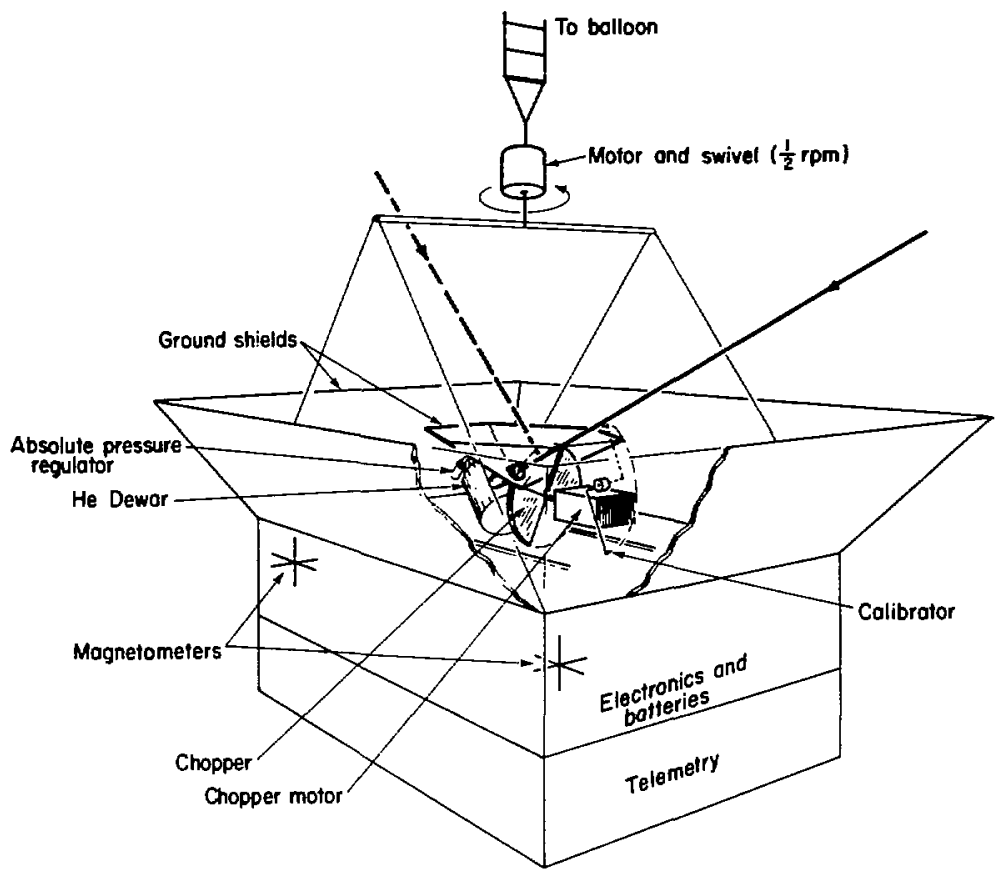

Figure 2.7. Perspective view of gondola in April 1982 configuration. Gondola dimensions are $0.8 \times 0.9 \times 1.7 \mathrm{~m}$. 
the desired altitude. With its various confgurations and hosts, this experiment bas flown with balloons ranging from sis hundred thousand to six million cubic feet in volume. Hanging under the balloon, and connected to it by a termination fitting which is separated at the end of the fight, is the parachute. The rotor and gondola are attached to the parachute at the end of a wire cable ladder long enough to keep the balloon 30 degrees away from the radiometer beams. Figure 2.8 shows the balloon and flight train. 


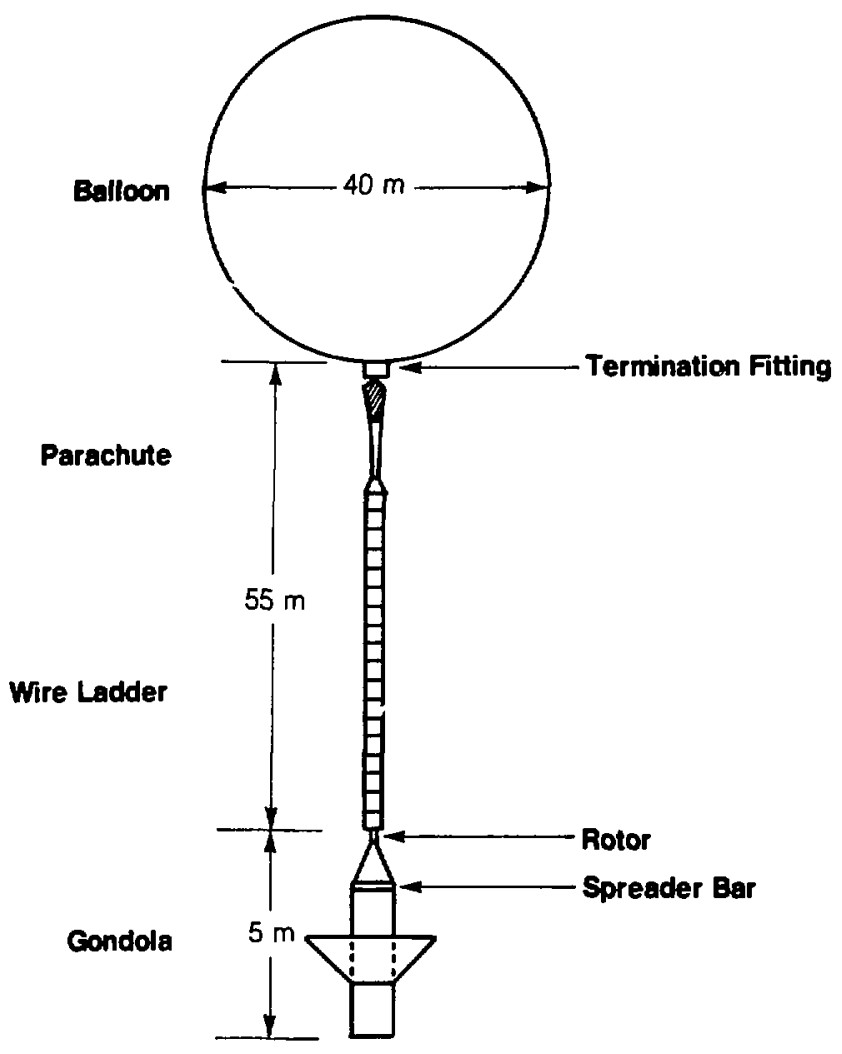

Figure 2.8. Balloun and flight train for April 1982 flight. Not to scale. 


\section{Chapter 8 \\ System Operation}

\subsection{Radiometer Performance}

\subsubsection{Noise Temperature}

The noise temperature of the radiometer at ambient temperature, in wideband measurements over the full $540 \mathrm{MHz}$ IF bandwidth, is $650 \mathrm{~K}$. At liquid nitrogen temperature, the noise temperature drops to $220 \mathrm{~K}$, and at its normal operating temperature of $4 \mathrm{~K}$ the radiometer system temperature is $135 \mathrm{~K}$. Over the IF bandwidth, this yields a system sensitivity of $12 \mathrm{mK}$ for one second net integration time.

\subsubsection{Calibration}

The radiometer is calibrated by placing an ambient temperature blackbody emitter ill one beam and a blackbody target saturated with liquid nitrogen in the other. The calibration constant (Kelvins antenna temperature per volt) changes by a factor of two between operation at. $300 \mathrm{~K}$ and at $4 \mathrm{~K}$. At $4 \mathrm{~K}$, t'se system is quite stable, with the RMS scatter in widely separated calibrations throughout a year of $2 \%$. Relative calibrations of the system during a flight are 
provided by inserting a small blackbody into one of the radiometer beams every 27 minutes. The calibration sequences last 48 seconds, causing a $3 \%$ loss of potential observation time. Further details of system calibration and corrections are presented in Appendix C.

\subsubsection{Operating Parameters}

Several factors determine the operating parameters of this apparatus. The chopping frequency of $23 \frac{2}{3} \mathrm{~Hz}$ is high enough to be in the region where thermal fluctuations with a flat spectrum dominate over fluctuations having the $1 / f$ spectrum typical of gain variations. These drifts and gain fluctuations limit the gondola rotation period, which must be short enough so that the drifts are small compared to the minimum detectable signal over the same interval. At the same time, to preserve sensitivity to small-scale structure, the antenna fleld of view should not be unnecessarily broadened by gondola rotation. The boxcar integrator averages over 50 chopper cycles $(2.11 \mathrm{sec})$ during which each beam scans an azimuthal range of 13 degrees at a rotation rate of 1 RPM. For one minute observation time, the radiometer sensitivity is $1.7 \mathrm{mK}$.

\subsubsection{System Testing}

In the laboratory, we investigated possible sources of systematic error by observin $\_$; stable load with the radiometer, modulating some parameter, and looking for a synchronous modulation in the radiomfier output. However, testing to the $0.1 \mathrm{mK}$ level on the ground is not feasible. Eleven hours of integration are necessary to reach that sensitivity with a $75 \mathrm{~K}$ target and over forty-flve for an ambient temperature load. Instead, useful tests involved modulating some parameter (e.g. magnetic field) at a higher leve' than was expected in fight. The limit on the instrument's response was then scaled assuming a linear stimulus/response relation. 


\subsubsection{Magnetic Dependence}

Since the radiometer rotates in the earth's magnetic field, any magnetic dependence of the output will yield a signal synchronous with gondola rotation and therefore will mimic background radiation anisotropy. With the isolator removed, there are no magnetically sensitive ele nents in the radiometer and no magnetic dependence was expected. Magnetic sensitivity was bounded by measuring a response of $0.4 \pm 1.9 \mathrm{mK}$ to a switched 10 gauss fleld. This puts a 0.1 mis limit to the effects of the $\mathbf{0 . 5}$ gauss peak-to-peak modulation of the horizontal component of th ' earth's magnetic fleld caused by gondola rotation.

\section{3. .4.2 Radic interference and Other Tests}

Sensitivity to radio interference was tested, particularly after data from first flight was st erely affected by it, by transmitting near the apparatus. Sources of radio frequency (RF) leskage were located and shielded by adding flters or enciosing components within RF-tight sl elds. To insure proper system operation under conditions expected at altitude, the system was placed in a thermal/vacuum chamber which was evacuated and cooled. This provided a "zeroth order" the ground were exacerbated by inability to access the system or to calibrate while the chamber was evacuated.

\subsection{Data Collection}

\subsubsection{Flight Scheduling}

The sun is a $6000 \mathrm{~K}$ source which, when diluted in the radiometer field of view, must $\varepsilon$ ill be attenuated $50 \mathrm{db}$ to contribute less than $0.1 \mathrm{mK}$. To achieve this rejection, it must be more taan 35 degrees of the beam axis. Sunlight might also cause temperature cycling synchronous 
with the gondola rotation. As a result, the radiometer collects useful dats primarily at night. If coverage of a particular portion of the sky is important, flights can be scheduled only for that part of the year when the desired section is visible at night.

The moon can contribute a signal of up to $800 \mathrm{mK}$ depending on lunar phase and position relative to the beam. To keep its effect under $0.1 \mathrm{mK}$, it must be more than 25 degrees off the beam axis. Depending on the moon's elevation, a significant amount of observation time will be lost in satisfying this requirement. On the other hand, the moon's signal is a valuable check on the radiometer pointing and can in principle provide an absolute calibration. In practice, inaccuracy in determining its position relative to the radiometer beam and the corresponding uncertainty in antenna gain prevented using the moon as an absolute calibrator.

There are two flight opportunities on either side of new moon, each of two or three days length, where the moon will be visible for a short period of time at the beginning or end of a flight. The determining factor in flight scheduling, though, invariably becomes the weather. Given the requirements on high-altitude wind speed and direction (particularly relative to the nearest large body of water), surface conditions at launch, and expected weather in the down-range recovery area, one rarely tas the luxury of waiting for ideal celestial alignmedts.

\subsubsection{July 1981 Flight}

The $90 \mathrm{GHz}$ radiometer was originally designed to fly in a gondola operated by David Wilkinson's group at Princeton University. This gondola, used to make the measurements reported in Cheng et al. (1979) and Boughn et al. (1981), had carried radiometers operating at. 24.8, 31.4, and $46 \mathrm{GHz}$. The Berkeley radiometer replaced the $24.8 \mathrm{GHz}$ Princeton radiometer. The gondola rotated at a rate of 1 RPM, and the Princeton magnetometers provided orientation information for both Princeton and Berkeley experimenis. 
Athongh presenting some of the inevitable difmculies of collaborating with another group, this gondola-sharing arrangement made my introduction to ballooning much easier. We at Berkeley were responsible for the $90 \mathrm{GHz}$ radiometer; the balloon, gondola, and light procedures were for the most part bandled by the Princetonians.

In June, 1981, we packed the radiometer and all of our test and support equipment into the back of a station wagon and drove to the National Scientific Balloon Facility in Palestine, Teras. We tested our equipment in the NSBF thermal/vacuum chamber mentioned previously, mounted the radiometer on the gondola, fed the Princeton magnetometer signals into our inflight recorder, and arranged for our radiometer output to be telemetered to the ground. We then checked for interference between the Berkeley experiment, the Princeton radiometers, and the NSBF-supplied command and telemetry equipment.

This last te st revealed a significant problem. The telemetry downlink transmitter at $1.485 \mathrm{GHz}$ fell in the middle of the $90 \mathrm{GHz}$ radjometer's IF band and severely interfered with the radiometer's operation. Deploying the transmitting antenna on a long cable under the gondola, and adding more shielding, did not reduce the interference to acceptable levels. In addition to transmitting the Princeton data, the transmitter was required by the NSBF to command and locate the balloon and could not be turned off.

On July 1 , weather conditions and the moon position were acceptable for launch. Taking advantage of the lull in surface winds at sunset, the balloon was launched at 19:13 Central Daylight Time (00:13 Univeral Time on July 2) and rose to float altitude of $25 \mathrm{~km}$ (25.1 millibar pressure) in 80 minutes. RF (or more precisely IF) interference was evident in the $90 \mathrm{GHz}$ radiometer data which was being telemetered to the ground. The in-flight calibrator also failed to operate during the first half of the flight, due (it was discovered later) to an overheated relay.

The gondola drifted almost due west at about 40 knots during the night. As it neared mountajnous terrain in west Texas the next morning, the fight was terminated, introducing 
some fortber problems. When the parachute opened, the spreader bar separating the gondola suspension cables from each other snapped. Possibly as a result, the paclage fipped over on its back upon landing. The radiometer was checked out after the experiment was recovered and returned to the balloon base. Except for the chopper motor, which had burned out when the chopper wheel jammed on impact, the radiometer functioned normally.

\subsubsection{November 1981 Flight}

\subsubsection{Modifications}

Based on the experience gained from the July flight, several modiflcations were made to the apparatus. Most importantly, a notch filter providing over $70 \mathrm{db}$ rejection at the telemetry transmitter frequency of $1485.5 \mathrm{MHz}$ was placed immediately before the detector diode. The isolator between the first and second IF ampliflers, which had been one of the most RF-sensitive components and whose presence was not essential to system operation, was removed. The second IF amplifler, bandpass filter, notch filter, and detector diode were moved into an RF-tight enclosure mounted directly on the dewar. All lines to this enclosure, as well as those going directly to the dewar, entered through filters.

The radiometer found a new host with Rainer Weiss from MTT, who was planning flights from Palestine in the fall. His four-channel, far-infrared bolometer system was to fly then in two conflgurations: with wide-beam (seven-degree FWHM) horns for a large-scale anisotropy experiment, and with two-degree beams to search for far-infrared sources. The MIT gondola required some additional modiflcations to be made to the $90 \mathrm{GHz}$ experiment. Although primary magnetometer signals were obtained from the MT experiment, a backup set of three orthogonal Hall probe magnetometers was also flown. The lithium batteries powering the system for the July flight were replaced by gelled-electrolyte lead-acid cells to permit system testing in the 
Eight configuration. Although mach lighter, the lithium batteries were not reehargable and condd not be used to power the system during ground tests. Other changes included surronnding the window over the antenna with a perforated collar through which nitrogen gas was exhausted to prevent frost formation on the window. These modiflcations were all done in Berkeley; the problems of mounting the experiment onto the MTT gondola and interfacing with MIT and with NSBF were left as fleld exercises.

In early October, 1981, the radiometer and associated equipment were once again packed into a station wagon for the three-day drive to Palestine. On arrival, the equipment was checked out and a structure for mounting it onto the MIT gondola was developed and built. The Berkeley equipment was placed above the MIT apparatus on two platforms projecting out the sides not occupied by MIT optics. The end result was a package $3 \mathrm{~m}$ high and extremely top-heavy. The hexagonal groundshield surrounding the $90 \mathrm{GHz}$ radiometer was extended on each face by adding foil-covered styrofoam panels. After waiting a month for weather, flights of other groups, and the completion of the the flrst MT flight (which was done with the same gondola), the $90 \mathrm{GHz}$ anisotropy experiment was ready to fly.

\subsubsection{Flight}

At sunset on November 4, 1981 (23:42 UT), the gondola was launched with a 6 million cubic foot balloon. The length of the parachute and flight train of $120 \mathrm{~m}$ gave the balloon an angular radius of 14 degrees at altitude when viewed from the gondola suspended underneath. Gondola rotation with a 74-second period was started fifteen minutes after launch. At this rate, each beam swept through 10.3 degrees in azimuth per 2.11-second integration period. The balloon rose more slowly than expected and never reached the $35 \mathrm{~km}$ altitude required by the MT infrared experiment. It did reach $27.5 \mathrm{~km}$ (17.1 mbar pressure) two hours after launch and peaked at $30 \mathrm{~km}$ (11.8 mbar) another half-hour later. Slowly dropping for the remainder of the 


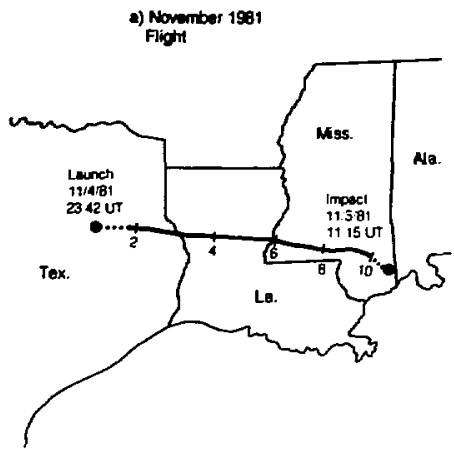

Ascent or Descent

Float b) April 19.2

Flight

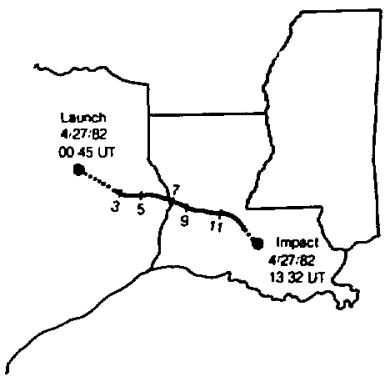

Tick marks Labelled in Hours UT

Figure 9.1. November 1981 and April 1982 flight paths.

flight, the balloon crossed $27.5 \mathrm{~km}$ again six hours after the peak altitude was reached. Two hours after that, the flight was terminated from an altitude of $26 \mathrm{~km}$ as the gondola neared the Gulf of Mexico, and the package landed in a wooded area near Mobile, Alabama. The fight path is shown in Figure 3.1a.

The Berkeley experiment worked well throughout the flight. As the gondola reached float altitude, the moon reached its maximum elevation of 39 degrees. six degrees off the beam. Becanse of contamination by the moon, one-quarter of the data taken in the flrst two hours at float had to be dropped from later analysis. On the ground, passing the telemetered lockin 
ampliater output through a 55-second time constant RC slter elearly showed the 3 mK dipole amisotropy in real time as a modulation synchronous with the gondola rotation. Figure 3.2 shows an equivalent plot from the April 1982 flight.

Analysis of the twenty in-flight calibrations showed that the gain at altitude varied by less than $\pm 0.7 \%$ (1 standard deviation). There were some periods of bighly irregular rotation during the flight, probably arising from shear winds acting on the asymmetric surface and mass distribution of the gondola.

No damage was incurred on landing, and the system was tested upon its return to Palestine. At ambient temperature (with no cryogens), the system gain was within one percent of its ambient tempersture pre-flight value. When the system was re-cooled following recovery, but had not yet reached equilibrium, the gain was within flve percent of the pre-flight cooled value.

\subsubsection{April 1982 Flight}

The November flight provided good data covering half the northern sky. Filling in the other half would increase the value of the data by much more than just the additional observation time, since complete coverage decouples otherwise highly correlated dipole and quadrupole parameters.

\subsubsection{Modifications}

To get this additional coverage, we made plans to fy again in April. The radiometer remained basically unchanged. What needed to be added was the equipment which had previously been supplied by our hosts - primary magnetometers, a gondola, and a balloon. A multiaxis fluxgate magnetometer provided the primary $x$ and $y$ reference, with the Hall probe mag- 
Signal

Magnetometers
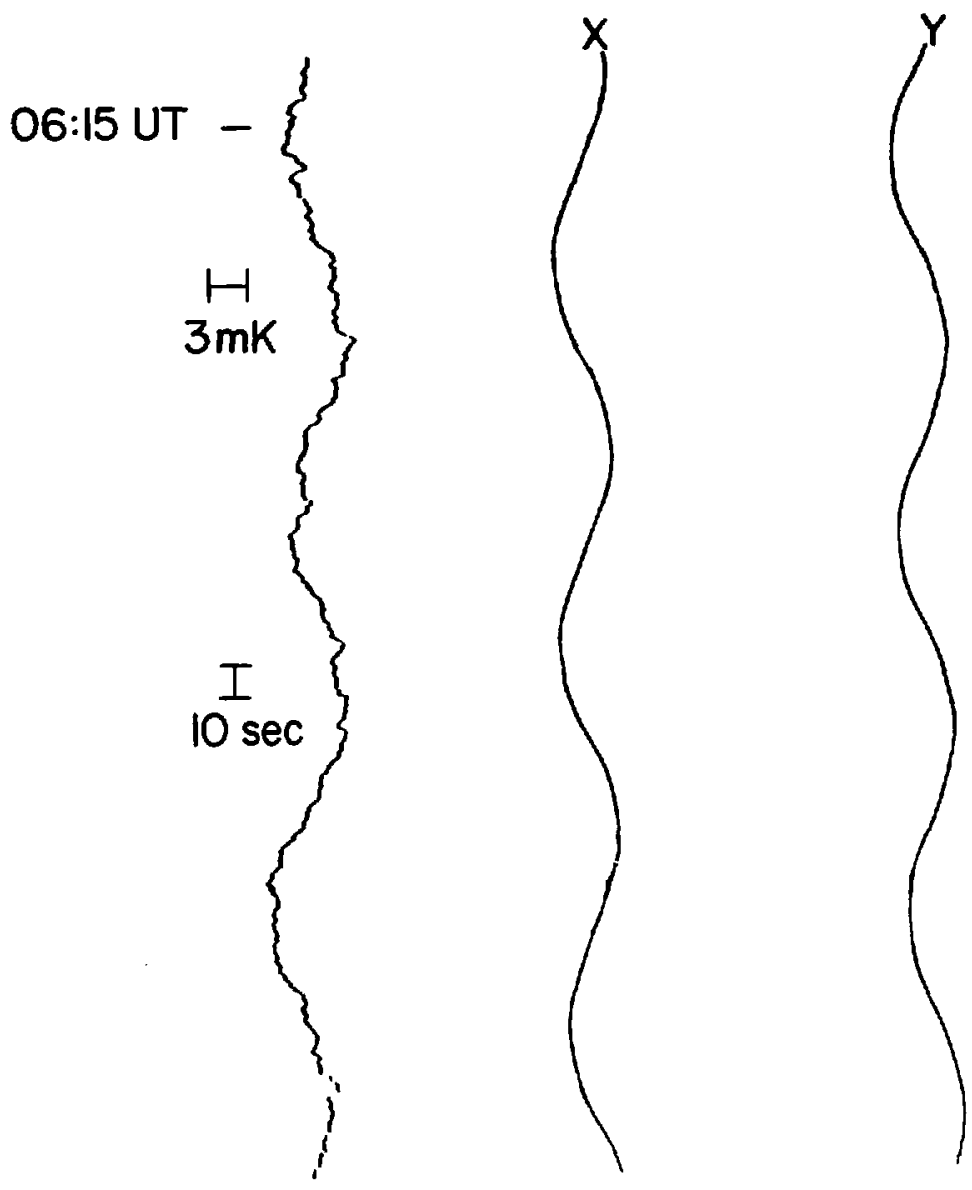

Figure 8.\&. Real-time dipole signal. 
netometers retained 25 s backup unit. A gondola, $0.8 \times 0.9$ × $1.7 \mathrm{~m}$ in size, was constructed and surrounded by an external aluminum sheet groundshield extending up to the height of the internal hexagonal shield. The gondola carried the radiometer, electronics, and batteries, and provided room for the NSBF telemetry package. Analysis of the radiometer's performance during the November flight showed that the gondola could be rotated more slowly, so the rotation was slowed down to a 110 second period for the April flight. At this rate, the radiometer beams swept through one beam-width (7.0 degrees) in one boxcar integration period.

\subsubsection{Flight}

Having obtained two balloons and authorization for flight, we packed the equipment. (no longer fitting in a station wagon) into a van and took the by-now-familiar route to Palestine. After checking out the equipment, rigging the gondola, and connecting our telemetry and telecommand lines to the NSBF telemetry package, we were ready to fly four days after arrival. With a small (one million cubic foot) balloon, our flight was not as restricted by launch surface weather conditions as those using larger balloons. The high altitude wind and downrange weather requirements, though, still had to be satisfled.

On April 26, the 350 pound gondola was launched at sunset at 19:45 Central Daylight. Time (00:45 UT on April 27). The gondola hung $55 \mathrm{~m}$ below the balloon, which had an angular radius of 16 degrees at altitude. The rotor was turned on just after launch. The gondola reached its maximum altitude of $28.5 \mathrm{~km}$ (14.6 mbar pressure) two hours ather launch; it dropped very slowly after that but remained above $27.5 \mathrm{~km}(17.1 \mathrm{mbar})$ for the duration of the flight. The moon, setting as the instrument rose, was within 25 degrees of the beam for less than one-half hour after the balloon reached float altitude and did not contribute a signal strong enough to see above the radiometer noise.

Gondola rotation was more uniform than it had been in November, and the CBR dipole 
anisotropy was again observable in real-time in the downlinked radiometer ontput (Figure 3.2). The rop-up calibrator rose every 27 minutes, as expected. However, the calibration signals were extremely erratic, varying by much more than would be consistent with the stability of the radiometer offset. Examination of the calibrator after recovery showed that the arm was severely bent, presumably having been caught just after launch by some of the tape fastening thermal insulation to the chopper motor assembly and causing it to rise to irregular positions during the flight.

The gondola drifted east during the night, taking a southward turn eleven hours into the flight near sunrise. Wanting to keep the gondola out of the Gnlf of Mexico even more than we wanted to check our pointing with the sun, we terminated the flight just after sunrise near Baton Rouge, LA. The April ground track is shown in Figure 3.I b; sky coverage obtained from both November and April flights is shown in Figure 3.3.

After the apparatus had been recovered and returned, it was cooled and tested. Postflight calibration was within $3 \%$ of the pre-flight value. Eight days after arrival, we were on the road back to Berkeley.

\subsubsection{November 1982 Flight}

With two successful Texas flights providing coverage of most of the northern sky, southern sky observations became extremely attractive. In addition to decoupling the axisymmetric dipole and quadrupole terms which cannot be separated in data taken from a single declination (see Chapter 5), southern hemisphere measurements permit observations of the galactic center. Dust emission should be maximized there, making possible either its measurement or the establishment of a firm upper limit to the galactic dust contribution. Additional motivations for further flights were the increased observation time and the chance to modify and re-fy the in-fight calibrator. 


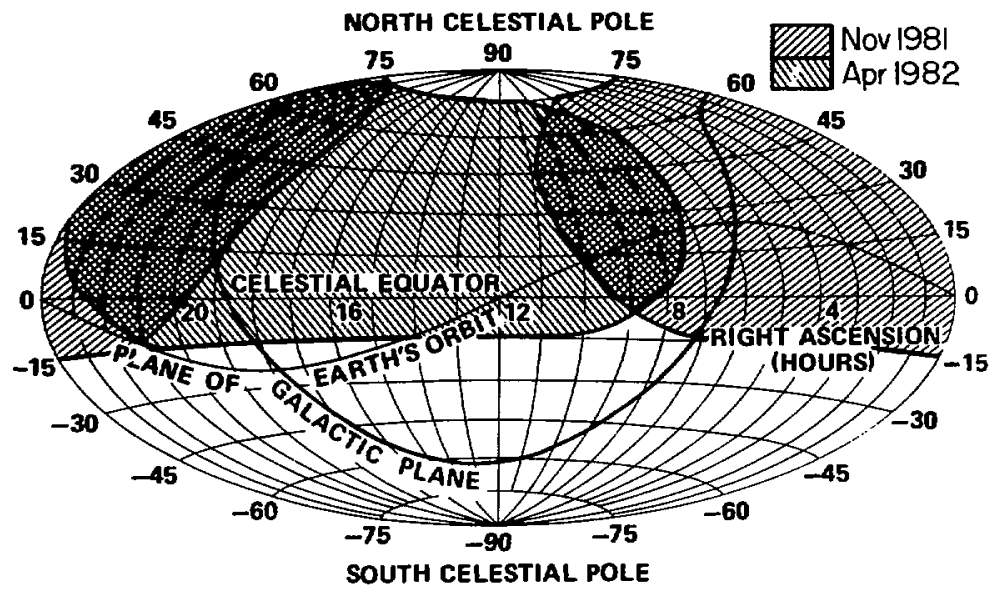

Figure 9.ง. Northern sky coverage. Hammer-Aitoff equal area projection. 


\subsubsection{Modifications}

The existing in-flight calibratos obsermations placed limits on gain variations during a Ilight. More usefdl would be a foll-beam calibrator which could provide an absolute calibration of the radiometer at altitude to relate the radiometer's ground performance to its fight operation.

A blackbody target $10 \mathrm{~cm}$ by $10 \mathrm{~cm}$ in ares with a front surface of cones $2.5 \mathrm{~cm}$ bigh was constructed at NASA's Goddard Space Flight Center out of iron-loaded epoxy. The target surface, including the valleys betmeen the cones, was covered with styrofoam to keep the emitting surface at a stable temperature. Reflection off the target's front surface, minimized by the shape of the cones and by the high absorption of the epary, was suppressed by more than $24.5 \mathrm{db}$. The target was incorporated into a new calibrator arm and the lockin amplifler circuitry was modified to automatically reduce system gain by a factor of 100 when the target was inserted into one beam.

At the same time, we had been making arrangements with the Brazilian Institute for Space Research (Instituto de Pesquisas Espacias - INPE) to use their ballooning facilities. We modifled the gondola slightly to accept the Brazilian telemetry system, and in late October, 1982, we air-freighted the equipment, two balloons, and 500 liters of liquid helium to INPE headquarters near Saõ Paulo, Brazil.

\subsubsection{Flight}

We ourselves arrived in early November to find our equipment and the helium (a signifleant addition to that nation's supply) in good shape. After a week's preparation and coordination, we moved to the launch facility at Cachoeira Paulista (latitude $23^{\circ}$ South) $100 \mathrm{~km}$ angy where the INPE flight crew rigged the gondols and fight train and prepared for launch. Three launch attempts were serubbed over the nert several days due to weather conditions or 
telemetry problems, but at 17:14 local time (20:14 UT) on November 20, 1982, the gondola was lannched. The 1.75 million cubic-foot balloon reached float altitude of $30.5 \mathrm{~km}$ two hours later.

Observed on the ground, the limited number of signals which the INPE telemetry could downlipk indicated that the instrument was performing quite well. The moon, which passed through the center of the radiometer beams due to the later-than-desired launch date, was highly visible in data at the beginning of the flight, and the dipole anisotropy was clearly evident in real-time throughout the night. The balioon's altitude at float remained between 30 and $31 \mathrm{~km}$. Fourteen hours after launch, the sun rose into the radiometer beams. After ohserving it for a few rotations to check the magnetometer alignment, we powered down the radiometer and gave the command to release the gondola from the balloon. Nothing happened. A long hour later, it became clear that despite four explosive bolts with redundant command circuits and power supplies, the termination fitting remained connected. The balloon and gondola stayed aloft that day, losing altitude and flnally drifting out of celemetry range the following night.

Aerial searches for the signal from the on-board radio beacon were hampered by bad weather and by poor position fises for the balloon's last known locations. The searches did not locate the gondola during the beacon's three-day lifetime. Subsequent searches and reward offers (Figure 3.4) were also unproductive, and the gondola has not been recovered.

\subsubsection{Post Mortem}

The down-linked analog telemetry data, intended for real-time monitoring (or worry) but not for data analysis, is of questionable quality. The onboard voltage-controlled-oscillators apparently drifted considerabjy, and much telemetery was contaminated with dropouts and interference. Nevertheless, downlinked data was recorded on the ground and has since been digitized. Some useful engineering data has been obtained from the Brazil flight, including limits 
Cr\$450.000,00 (QUATRUCENTOS E CINCOENTA MIL CRUZEIROS)

PAR: INFERMACOES QUE LEVEM $A$ RECUPERACAO DE UMA CARGA CIENTIFICA EM BALKO.

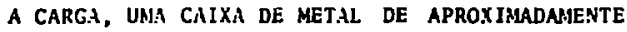
IM $\times 2 M \times 1 M$, DEVE TER CAIDO APROXIMADAMENTE EM 26 DE NOVEMBRO DE 1982 NA REGIAO PROXIMA DE CORNELIO PROCO PIO, hostrada No MaPs EM aNeXo.

JUNTO COM a EXPERIENCIA DEVE ESTAR UM PARAQUEDAS COR LAPANJA BRILHANTE E PODE SER VISTO DO AR.

PEDAÇOS DO BALJO, DE PLASTICO BRANCO, DEVEM ES TAR POR PERTO.

PARA MAIORES INFORMACOES ligue, A COBRAR PARA:

OR I M MARTIN

INSTITUTO DE PLSQUISAS ESPACIAIS - INPE

CAIXA POSTAL 515 - 12.200 S J DOS CAMPOS - SP

TELEFONE: (0123) 22-9977

A QIJALQUER HORA DO DIA OU NOITE

Figure 9.4. Reward notice for gondola. 
on pointing accuracy. The reward for information leading to recovery of the onboard data cassette is still outstanding. 


\section{Chapter \&}

\section{Data Reduction and Processing}

\subsection{Data Format}

The boxcar-integrated radiometer output and the horizontal magnetometer values were recorded on the on-board tape cassette every 2.1 seconds. Every 6.3 seconds, the Hall probe magnetometer values were written, and sirteen housekeeping siguals were recorded once in every 25 second (12 integrator output) record along with Universal Time and some digital status bits. Gondola altitude and position, determined by a pressure transducer and an on-board OMEGA navigation receiver, were recovered after the flight from the dow nlinked telemetry data.

In Berkeley, the data on the flight cassette was read and transferred to disk. Absolute caljbrations done before flight and resorded by hand were cross-checked with the values written on pre-flight and post-flight tapes. For tht November flight, in-flight calibrations done every 27 minutes (64 records) were tabulated and remored from the data. For the April flight, since the in-flight calibrator was damaged socn atter launch, observations were removed from the data but. could not. be used for relative calibration. 


\subsection{Beam Positional Reconstruction}

The gondola position and orientation, obtained from magnetometer and tracking data, uniquely determine the position of each beam on the sky. The fluxgate $x-y$ magnetometer ralues, recorded just after each boxcar-integrated radiometer value, were interpolated to times corresponding to the midpoint of the integration period. The midpoint and amplitude of the magnetometer signals were found from twenty-two minute running averages of the magnetometer extrema. Removing the DC level from the magnetometer signals and normalizing their amplitudes yielded the sine and cosine of the gondola azimuth angle with respect to the horizontal component of the earth's magnetic field. Correcting the magnetic azimuth by the deviation between magnetic and true north, obtained from National Oceanic and Atmospheric Administration sectional navigation maps, yielded the true azimuth angle.

Observations of the moon at the beginning of the Norember flight showed that the beam azimuth angles were within 1.5 degrees of the values determined by the above process. Although data recovered from telemetry from the Brazil flight was not put through an identical analysis, observations of the sun at the end of the flight showed the deviation between magnetometerderived azimuth and true azimuth to be $0.2 \pm 0.8$ degree for the direct beam and $0.9 \pm 0.8$ degree for the reflected beam.

Gondola latitude and longitude were determined by the onboard OMEGA receiver and logged on the ground every five minutes. Comparison of the OMEGA positions with the launch and impact locations and with FAA radar flxes showed that the OMEGA positions were accurate in April and at the beginning of the November flight but gave a position 0.6 degree too far east. and 0.5 degree too far north for the November impact point. The November positions were corrected for this offset by assuming the error in position was proportional to the distance from Palestine. 
For both Teras data dights, the gondola drifted almost due east at a fairly uniforn rate. For data analysis, a lipear ground track with constant speed was assumed. This approximation was at all times within 0.5 degree of the OMEGA position (corrected, in the Norember case, for the position offet). The gondola location and azimuth were then used to reconstruct the sky position of each beam.

\subsection{Data Editing and Sky Coverage}

Data taken during calibration sequences, along with data taken when either radiometer beam pointed within 25 degrees of the moon, was removed. For the November flight, the moon cuts eliminated $25 \%$ of the first 2.3 hours at float or $6 \%$ of the total November data. Less than $0.2 \%$ of the April data was removed due to the moon. An additional twenty-minute section of data two-thirds of the way through the April flight, during which the offset changed by four $m K$ and then returned to its previous value, was deleted. During that section, the RMS difference between consecutive 25-second data averages was more than twice the RMS difference between consecutive 25-second arerages for the rest of the data. The RMS difference between consecutive 2.11-second integrator values in that section is not noticeably higher than in other sections, indicating that the excess noise in the deleted section is at longer time scales than 4 seconds. No other section of data showed similar behavior. Removing the section had negligible effect (less than $15 \%$ of the one-standard-deviation statistical error) on spherical harmonic coeffients fitted to the data. No additional non-statistical fluctuations were seen, and after the moon cuts, none of the 29000 radiometer 2.11 -second boxcar integrator values differed from a local mean by more than 4.5 standard deviations.

Figure 4.1 show s the density of sky coverage of all data remaining after the mooi cuts and the twenty-minute cut descr. hed above. Of the data remainisg after the moon cuts, $17 \%$ of the November data and $10 \%$ of the April data had one of the radiom rter beams within 5 degrees 
of the galactic plane. These data, $13 \%$ of the total, were excluded from some of the spherical harmonic ats discussed in the following chapter. The galactic plane is indicated by Figure 5.3 in the next chapter, which is done to the same scale as Figure 4.1.

\subsection{Ofiset Analysis and Removal}

As stated previously, the chopper wheel introduced an offset between direct and reflected beams of the radiometer. This offset had an average magnitude of $201 \mathrm{mK}$ in the November flight and $225 \mathrm{mK}$ in April, implying emissivities of $8.9 \times 10^{-4}$ and $1.0 \times 10^{-3}$ for a chopper temperature of $225 \mathrm{~K}$. The offset varied by no more $\pm 2.5 \%$ in November and $+2.5 /-5 \%$ in April (even with the deleted section discussed above left in). Offset variations, except for the deleted section, correlated fairly well with changes in the temperature measured by a sensor on the in-filght calibrator.

Figures 4.2 and 4.3 show the behavior of the radiometer offset for the November and April flights, respectively. The lower box in each figure shows the radiometar ouf put, including offset, averaged over 100-second intervals, and the upper box shows the calibrator temperature. With the lockin amplifier blanked while the chopper crosses the horn, each 100-second interval represents 80 seconds of net integration time. Moon-contaminated data are not included in the plots, but Figure 4.3 shows the twenty-minute section of the April flight deleted from later analysis. The smooth curve in the radiometer output plots is a ten-term Legendre-series fit to the ofiset.

To remove the offset, data were averaged in 6.5 -minute sections, one-quarter of the interval between calibrations. A piece-wise cubic spline through the averages was subtracted from the data, removing low-frequency drifts but not affecting signals at the gondola rotation frequency (0.81 RPM in November and 0.55 RPM in April). After the section in the April flight discussed above was deleted, residual offset for each 3.3 minute segment of data (half the 6.5 

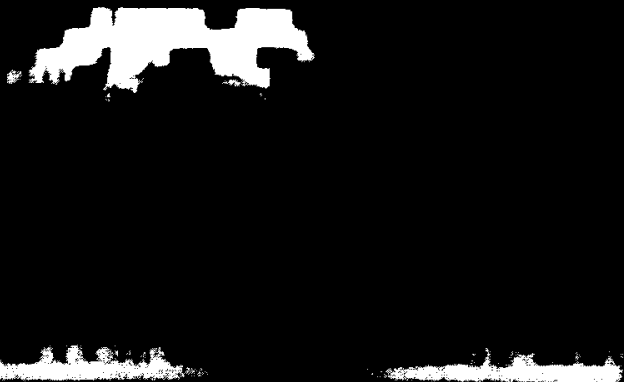

Figure 4.1. Sine equal-area projection of the number of observations per pixel. Observation time equals 2.11 seconds times the number of observations. Declination of displayed region ranges from $-15^{\circ}$ at the bottom to $+80^{\circ}$ at the top: RA decreases from 24 hours at left to 0 hours at right. Pixels are $5^{\circ}$ high in declination $\delta$ by $3^{\circ} / \cos \delta$ wide in RA. having equal areas on the sky of 15 square degrees. 


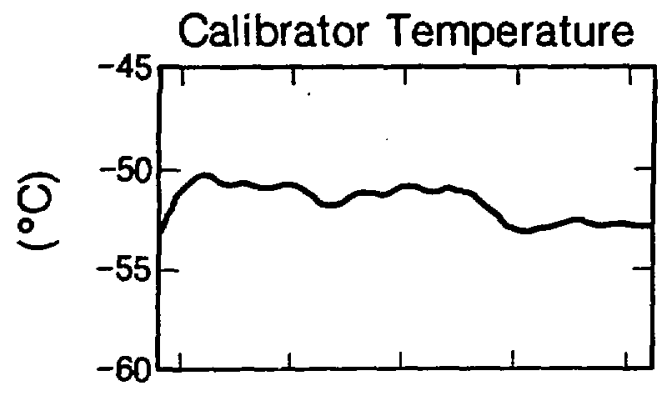

\section{Radiometer Output Including Offset}

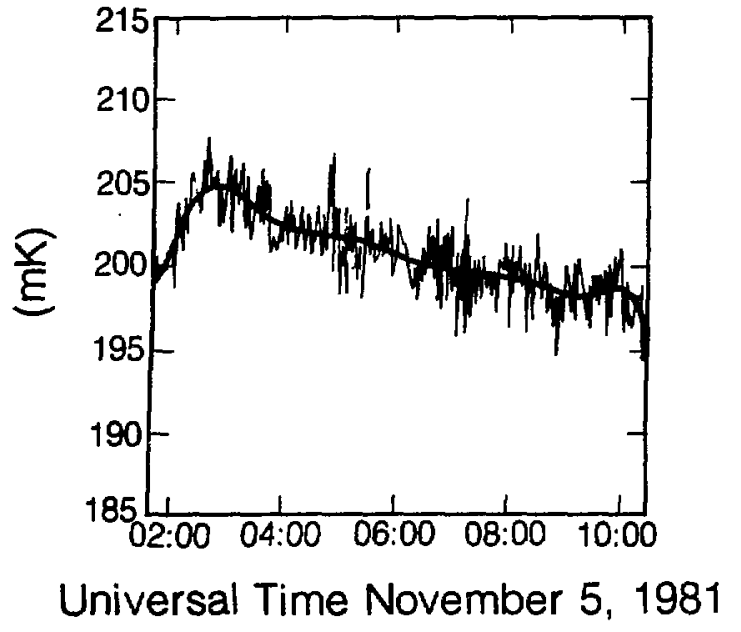

Figure 4.\&. November 1981 Flight: Lower flgure is radiometer offset in $\mathrm{mK}$, averaged over 100-second intervals and excluding moon-contaminated data. Smooth curve is 10-term Legendre-series fit. Upper figure is calibrator temperature in degrees $C$. over the same period. 


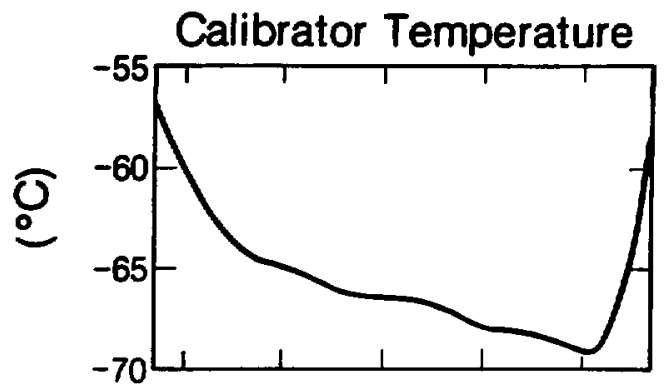

Radiometer Output Including Offset

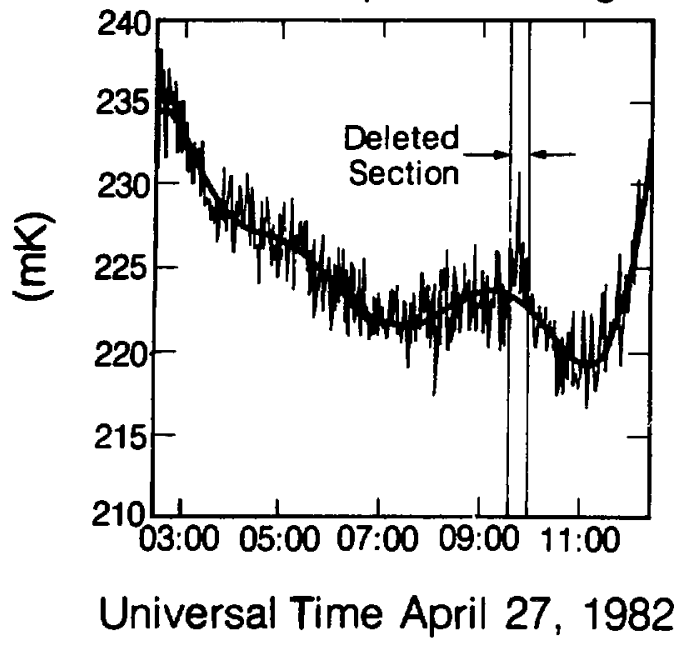

Figure 4.9. April 1982 Flight: Lower figure is radiometer offset in $\mathrm{mK}$, averaged over 100-second intervals and excluding moon-contaminated data but showing the twenty-minute segment deleted from later analysis. Smooth curve is 10-term Legendre-series fit. Upper figure is calibrator temperature in degrees $\mathrm{C}$. 
minute areraging interval) was less than $2 \mathrm{mK}$ except for three 3.3-minute segments of the April Alight which had residual oflsets under $3 \mathbf{m K}$.

The data analysis is insensitive to the technique used to remove the offet. Fitting and removing a 10-term or a 25-term Legendre series to the offset rather than a spline changes fited parameters by less than $25 \%$ of the statistical error. Since the total variance of the data set after removal of a spline fit to the offset is less than the variance after removal of a Legendre series, the spline is a better approximation. Sky maps inverted from the data after a spline has been removed converge faster than when a Legendre series is used.

Although no sensor directly measured the temperature of the chopper wheel, an upper limit to the variation in chopper temperature synchronous with gondola rotation can be found using measurements of the calibrator temperature. The calibrator's small thermal mass and more exposed location should make it more sensitive than the chopper to synchronous temperature modulation. The temperature sensor on the in-fight calibrator survived whatever happened to the calibrator itself early in the April flight, so temperature variation could be examined. Synchronous variation of the calibrator temperature was less than $0.03^{\circ} \mathrm{C}$ at all times. Giving the chopper a synchronous variation of this amplitude would generate a spurious anisotropy signal of less than $0.03 \mathrm{mK}$.

\subsection{Conversion to Antenna Temperature and Correction to Barycenter}

After the data edits had been made and the offset removed, the radiometer output values were multiplied by the system calibration to yield the antenna temperature difference between beams. As is discussed further in Appendix C, the same calibration value was used for both flights, and observations of the in-flight calibrator in November indicated that this value did not change during a flight. Ohservations were referred to the solar system barycenter by subtracting from each data point the temperature diffesence generated by the earth's orbital 
motion, assuming a blackbody CBR with a temperature of $2.7 \mathrm{~K}$. The marimum $\mathbf{v}$ lue of this correction to either radiometer beam is $0.22 \mathrm{mK}$, and with the 90 degree opening angle between beams the maximum adjustment to their temperature diference is $0.31 \mathrm{mK}$. Therefcre, a $30 \%$ error in this correction would change any data point by less than $0.1 \mathrm{mK}$. 


\section{Chapter 5}

\section{Data Analysis}

\subsection{Spherical Harmonic Fits}

\subsubsection{Results}

The radiometer output is a measurement of the temperature differeace between two parts of the sky. The most straightforward way to 8nalyze the differences is to do a least-squares fit to a spherical harmonic multipole expansion. Table 5.1 gives the basis functions chosen, and the fitting procedure is described in Appendix $D$.

The best-fit dipole and quadrupole terms, excluding data taken less than five degrees from the galactic plane, are given in Table 5.2. The galactic cut eliminates $17 \%$ of the November data and $10 \%$ of the April data, or $13 \%$ of the total. The fitted terms change by less than one statistical standard deviation when data near the galactic plane is inciuded (Table 5.6), and they are not signiflcantly affected by including a galactic model term in the fts (Table 5.7). Galactic contributions are diseussed further in Section 5.1.5.

The data from the two Texas fights analyzed in this work were all tajen at constant latitude. As a result, the axisymmetric quadrupole term $Q_{1}\left(\frac{3}{2} \sin ^{2} \delta-\frac{1}{2}\right)$ and the dipole term $T_{z} \sin \delta$ cannot be decoupled, as can be seen from the following argument: The difference in values 
Table 5.1. Spherical harmonic basis fanctions.

$$
T(\dot{n})=T_{0}+\vec{T} \cdot \dot{n}+\sum_{i=1}^{5} Q_{i} \cdot g_{i}(\dot{n})
$$

$\delta$ is declination measured north from celestial equator

$\alpha$ is right ascension measured east from 0

$\hat{n}$ is unit vector in direction of observation

\begin{tabular}{|c|c|c|c|}
\hline $\begin{array}{c}\text { Basis } \\
\text { Function }\end{array}$ & $\begin{array}{c}\text { Spherical } \\
\text { Harmonic }\end{array}$ & $\begin{array}{c}\text { Angular } \\
\text { Dependence }\end{array}$ & $\begin{array}{c}\text { Rectangular } \\
\text { Representation }\end{array}$ \\
\hline$n_{x}$ & $-\sqrt{\frac{2 \pi}{3}}\left(Y_{11}-Y_{1,-1}\right)$ & $\cos \delta \cos \alpha$ & $x$ \\
$n_{y}$ & $i \sqrt{\frac{2 \pi}{3}}\left(Y_{11}+Y_{1,-1}\right)$ & $\cos \delta \sin \alpha$ & $y$ \\
$n_{z}$ & $\sqrt{\frac{4 \pi}{3}} Y_{10}$ & $\sin \delta$ & $z$ \\
$q_{1}^{*}$ & $\sqrt{\frac{4 \pi}{5}} Y_{20}$ & $\frac{1}{2}\left(3 \sin { }^{2} \delta-1\right)$ & $\frac{1}{2}\left(3 z^{2}-1\right)$ \\
$q_{2}$ & $-2 \sqrt{\frac{\pi}{5}}\left(Y_{21}-Y_{2,-1}\right)$ & $\sin 2 \delta \cos \alpha$ & $2 x z$ \\
$q_{3}$ & $2 i \sqrt{\frac{2 \pi}{5}}\left(Y_{21}+Y_{2,-1}\right)$ & $\sin 2 \delta \sin \alpha$ & $2 y z$ \\
$q_{1}$ & $2 \sqrt{\frac{2 \pi}{5}}\left(Y_{22}+Y_{2,-2}\right)$ & $\cos ^{2} \delta \cos 2 \alpha$ & $x^{2}-y^{2}$ \\
$q_{5}$ & $-2 i \sqrt{\frac{2 \pi}{5}}\left(Y_{22}-Y_{2,-2}\right)$ & $\cos ^{2} \delta \sin 2 \alpha$ & $2 x y$ \\
\hline
\end{tabular}

of the $Q_{1}$ terin between beams at declinations $\delta_{a}$ and $\delta_{b}$ is $\frac{3}{2} Q_{1}\left(\sin \delta_{a}-\sin \delta_{b}\right)\left(\sin \delta_{a}+\sin \delta_{b}\right)$. For constant balloon zenith declination $\delta_{z}$ and radiometer opening angle $\theta$, the factor $\frac{3}{2}\left(\sin \delta_{a}+\right.$ $\left.\sin \delta_{b}\right)$ has a fixed value equal to $3 \sin \delta_{z} \cos (\theta / 2)=1.10$ for our latitude. The $Q_{1}$ differences are strictly proportional to the $T_{z}$ differences, and as a result, the $Q_{1}$ term was not included in multipole fits. The true $Q_{1}$ component of the anisotropy, if any, will have been incorporated into the fltted $T_{z}$ coefficient by the relation $T_{z}$ (fitted) $=T_{z}$ (true) $+1.10 Q_{1}$ (true). All references to $T_{\mathrm{r}}$ in this thesis refer to the fited $T_{z}$ ralue. 
Table 5.2. Global dipole-plns-quadrapole fit in $\mathrm{mK}$ antenna temperature.

Data within 5 degrees of the galactic plane has been excluded. All errors are statistical only.

\begin{tabular}{|c|c|c|c|c|c|c|c|c|}
\hline Term & Magnitude & \multicolumn{7}{|c|}{ Correlation Coeff cients } \\
\hline$T_{z}$ & $-2.760 \pm 0.084$ & 1.000 & -0.094 & 0.032 & -0.335 & 0.261 & -0.018 & 0.045 \\
\hline$T_{y}$ & $0.541 \pm 0.090$ & & 1.000 & -0.008 & 0.254 & -0.363 & -0.097 & 0.048 \\
\hline$T_{z}$ & $-0.252 \pm 0.076$ & & & 1.000 & 0.090 & 0.100 & 0.078 & 0.235 \\
\hline$Q_{2}$ & $0.118 \pm 0.102$ & & & & 1.000 & -0.126 & -0.053 & 0.007 \\
\hline$Q_{3}$ & $0.129 \pm 0.100$ & & & & & 1.000 & -0.033 & 0.161 \\
\hline$Q_{4}$ & $-0.050 \pm 0.069$ & & & & & & 1.000 & 0.055 \\
\hline$Q_{5}$ & $0.070 \pm 0.065$ & & & & & & & 1.000 \\
\hline & & & & & & & & \\
\hline
\end{tabular}

\subsubsection{Data Weighting}

Since the fluctuations in radiometer outpat throughout a flight are consistent with noise having constant variance, all data from a given flight are weighted equally. The data variance used for each flight is half the mean-squared difference between consecutive two-second integrated output values. The square root of this variance, proportional to the system noise temperature, was $10.0 \pm 0.1 \mathrm{mK}$ for the November flight and $9.9 \pm 0.1 \mathrm{mK}$ for April. The contribution of the dipole anisotropy signal to the RMS poirt-to-point differences is under $0.05 \%$. Once this contribution is removed, half the mean squared difference provides an accurate measure of the true variance of the data if the noise is white (equal power per unit frequency). If there is relatively enbanced power at longer time scales, the mean squared point-to-point difference underestimates the true variance.

The variance of the entire November data set after offset removal is $5.2 \pm 1.1 \%$ bigher than the variance determined from point-to-point differences; removing the dipole signal gives a variance $0.8 \pm 1.1^{\%}$ higher than that determined from the differences. For the April fligbt, the 
variance of the entire data set after ofiset removal is $8.8 \pm 1.1 \%$ higher than thet found from the differences. Removing the dipole signal accounts for about half of the excess, leaving a variance for the entire data set $3.1 \pm 1.1 \%$ greater than that computed from the point-to-point differences. This excess suggests some noise power with a period longer than 4 seconds remains even after the anomalous twenty-minute section (see Chapter 4 ) has been removed. If the weighting used for the April data is decreased by $3 \%$ to account for the excess variance, no fltted coefficient changes by more than $12 \%$ of the statistical error.

\subsubsection{Chi-Square Statistics and Hourly Arerages}

The $\chi^{2}$ value presented in Table 5.2 of 25811 for 25324 degrees of freedom (DOF) is not very meaningful. Taken literally, it represents a $2 \%$ probabilit that the data can be modeled by dipole and quadrupole coefficients aione. However, $\chi^{2}$ statistics with so many degrees of freedom are very sensitive to the values used for the formal ersors $\sigma_{i}$ of the individual data points. A $1 \%$ increase in the uncertainty assigned to each of the 25.00 observations lowers $\chi^{2}$ from 258]0 to 25301 and increases the confldence level from $2 \%$ to $54 \%$. While the $\chi^{2}$ values computed in the global flits may be used to compare one flt to a ther, they are not useful goodness-of-fit indicators in the absence of a very accurate determi,ation of uncertainty $\sigma_{i}$ of each observation.

By averaging the data in bins before fitting, the number of degrees of freedom is drastically reduced and the $\chi^{2}$ values are much less sensitive to the values used for the individualobservation errors. In addition, it is possible " $\mathrm{d}$ determine the uncertainties in each bin average from the scatter within the bin rather than fom some external estimate of the data uncertainty.

A convenient way to group balloon-gathered anisotropy data is to Fourier-analyze data gathered during some interval as a function of gondola azimuth angle $\phi$ (Corey 19i\&; Cheng et al., 1979). The dipole and quadrupole anisotropy can be determined from the cos $\phi$ (north - south) and $\sin \phi$ (east - west) coeffieients alone and will not contribute to $\cos n \phi$ or $\sin n \phi$ coefficients 
Table 5.8. Dipole-only parameters determined from hourly averages, in mK antenna temperature. Data within 5 degrees of the galactic plane has been excluded. Errors are statistical only.

\begin{tabular}{|c|c|c|}
\hline Term & Include all Data & $\begin{array}{c}\text { Exclude Final Interval } \\
\text { of Each Flight }\end{array}$ \\
\hline$T_{x}$ & $\begin{array}{r}-2.74 \pm 0.08 \\
0.55 \pm 0.08\end{array}$ & $\begin{array}{r}-2.75 \pm 0.09 \\
0.54 \pm 0.08 \\
T_{y} \\
T_{z}\end{array}$ \\
\hline $\begin{array}{c}-0.28 \pm 0.07 \\
\text { Chisquare/DOF }\end{array}$ & $46.42 / 39$ & $33.24 / 35$ \\
\hline $\begin{array}{c}\text { Confldence } \\
\text { Level }\end{array}$ & $19.3 \%$ & $55.3 \%$ \\
\hline
\end{tabular}

for $\mathbf{n}>1$. No anisotropy power of any order is contained in coefflcients with even $n$, since any celest:al signal must change sign as the gondola rotates $180^{\circ}$. The expressions relating the dipole and quadrupole parameters to the $\cos \phi$ and $\operatorname{sir} \phi$ coefficients and the radiometer opening angle are given in Appendix G.

The $90 \mathrm{GHz}$ data has been binned into 54-minute intervals (two calibration segments) for which $\cos \phi$ and $\sin \phi$ Fourier coefficients have been determined. The dipole-only anisotropy determined trom the Fourier coefficients is given $i_{s}$ Table 5.3, and the dipole-plus-quadrupole flt is presented in Table 5.4. Figure 5.1 shows the hourly sine and cosine coefficients. The curves in Figure 5.1 are the coefficients which would be generated by the combined dipole and quadrupole auisotropy in Table 5.4. The residual sine and cosine coefficients betreen the data and the dipole-only fit are show $\mathrm{a}$ in fig .re 5.2 along with smooth curves giving the difference between the dipole-plus-quadrupole fit and the dipole-only fit.

Since the individual two-second observations are spaced 7 to 10 degrees apart in azimuth angle, short segments of data do not provide good azimuthal coverage. As a result, Fourier 
Sine Coefficients

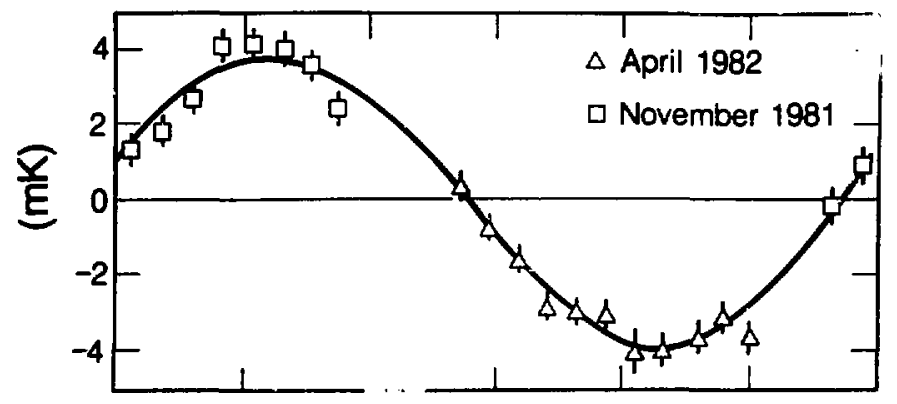

Cosine Coefficients

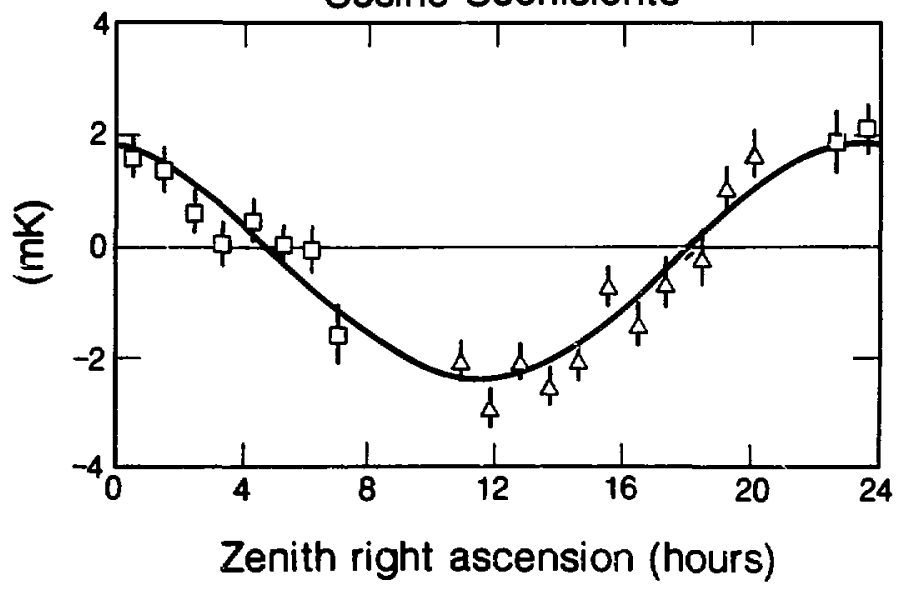

Figure 5.1. Hourly sine and cosine anisotropy coefficients. Data within 5 degrees of the galactic plane has been excluded. Curves are the sine and cosine terms corresponding to the dipole-plus-quadrupole fits in Table 5.4. 
Sine Coefficients
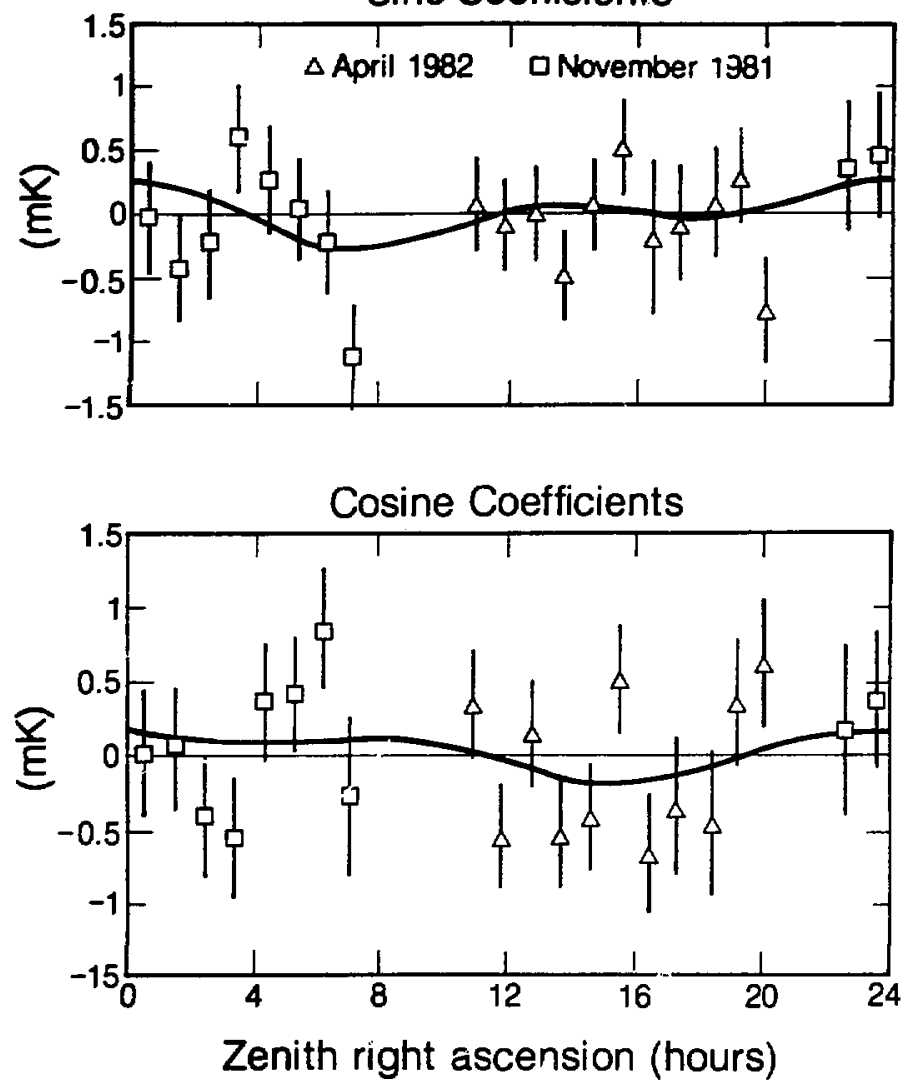

Figure 5.2. Points are residuals between hourly sine and cosine anisotropy coefficients and the dipole-only fit given in Table 5.3. Curves are the difference between dipole-plus-quadrupole (Table 5.4) and dipole-only (Table 5.3) fits. Data within 5 degrees of the galactic plane has been excluded. 
andyses were done for esch 54 -mingte internd in its eutirety. The errors of the hourly coeficients are not decermined from a scatter of individual coeficients, as mould be possibte if coefleients for many small intervals nere averaged, but instead represent statistical errors of the Fourier tits. The same individual-observation uncertainties used in the global spherical harmonic fits are also used to estimate errors in the Fourier coemeients. Since the fits in Table 5.4 have 35 degrees of freedom, as opposed to the 25324 in Table 5.2, the $\chi^{2}$ value in Table 5.4 is much less sensitive to small changes in the individual observation uncertainties.

T:it $\chi^{2}$ for the dipole-only fit in Table 5.3 is $\mathbf{4 6 . 4 2}$ for 39 degrees of freedom (19.3\% confidence level). Adding quadrapole terms (Table 5.4) reduces $\chi^{2}$ to 42.07 for 35 DOF without improving the conffdence level (19.1\%) and barely changing the dipole terms, showing that quadrupole terms are not compelle ' by the data. The value of $\chi^{2}$ is slightly higher than statistical error alone would be expected to generate. Essentially all the excess is contributed by the last intervals in both the November and April flights: the last November point (RA 7.09 hours) contributes a $\chi^{2}$ of 7.31 , and the last April point (RA 20.12 hours) contributes 5.78. This allocation of the chi-square motivated a search for systematic effects which could have influenced the ends, or the flights. Positional error should be minimized at the end of the flight since the impact point is well determined. Synchronous beating effects were not detected in the calibrator temperature sensor and would be highly unlikely in the November flight, which ended at a local solar time of 04:23 when the sun was 20 degrees below the horizon. At the end of the April flight (06:18 local solar time), the :un was 14 degrees above the horizon or 9 degrees above horizontal (the horizon at balloon altitude being 5 degrees below borizontal). The sun was therefore 36 degrees of the beam and should not have contributed a detectable signal unless diffraction over the groundshields has been grossly underestimated (see Section 5.3.1.5). The top one-half inch or so of the chopper wheel may have been directly illuminated at termination.

Fits were done excluding the last hourly coefflcients in each flight to test the sensitivity 
Table 5.4. Combined dipole-plus-quedrupole anisotropy determibed from bourly coefleients, in $\mathrm{mK}$ antenna temperature. Data within five degrees of the galactic plane has been excluded; errors are statistical only.

\begin{tabular}{|c|r|r|}
\hline Term & Include all Data & $\begin{array}{c}\text { Exclude Final Interval } \\
\text { of Each Flight }\end{array}$ \\
\hline$T_{x}$ & $-2.74 \pm 0.09$ & $-2.71 \pm 0.09$ \\
$T_{y}$ & $0.55 \pm 0.09$ & $0.49 \pm 0.10$ \\
$T_{z}$ & $-0.25 \pm 0.07$ & $-0.28 \pm 0.08$ \\
$Q_{2}$ & $0.11 \pm 0.10$ & $0.05 \pm 0.11$ \\
$Q_{3}$ & $0.12 \pm 0.10$ & $0.18 \pm 0.11$ \\
$Q_{4}$ & $-0.03 \pm 0.07$ & $0.03 \pm 0.07$ \\
$Q_{5}$ & $0.08 \pm 0.06$ & $0.02 \pm 0.07$ \\
\hline Chisquare/DOF & $42.07 / 35$ & $29.51 / 31$ \\
\hline Confidence & $19.1 \%$ & $54.3 \%$ \\
Level & & \\
\hline
\end{tabular}

of the fits to those points. The resultant parameters, given in the second columns of Tables 5.3 and 5.4, change by less than one statistical standard deviation. The $\chi^{2}$ for the dipole-only fit drops to 33.24 for 35 DOF, with the confidence level rising to $55.3 \%$. The dipole-plus-quadrupole $\chi^{2}$ becomes 29.51 for 31 DOF at the $54.3 \%$ confldence level. Although it is certainly not valid to exclude data simply because they generate large contributions to $\chi^{2}$, it is reassuring that the dipole and quadrupole parameters do not change signiflcantly when the possibly discrepant points are deleted. Since no unambiguous evidence of systematic error in the ends of the filghts could be found, all data were used in the global fits. Systematic error is explored further in Section i.3.

\subsubsection{Individual Flight Dipole Fits}

Each of the November or April flights, taken alone, covers about half the northern sky. 
Combined dipole-plus-gavadrupole its to data with such limited sky coverçe are highly correlated and are not very useful. Bowever, dipole-only its to each individual tight give meaningful results which are show in Table 5.5 along with the dipole-only fit to the two tights combined. Using the comparison analysis described in Appendix D, the difference between the two single-tight dipole fts is consistent with zero at the $21 \%$ confidence level.

\subsubsection{Galactic Effects and Models}

One of the prime motivations for measuring anisotropy at $90 \mathrm{GHz}$ was to minimize the galactic contribution. This hope appears to have been fulflled. Including or excluding the galactic plane has little effect on dipole and quadrupole fits, as is shown in Table 5.6. Galactic emission at $90 \mathrm{GHz}$ is thought to be dominated by thermal emission from interstellar dust. However, maps of the galactic dust distribution, inferred from infrared emission, have not yet (i.e. pre-IRAS) been made over large areas. Two models for galactic emission at $90 \mathrm{GHz}$, convolved over the radiometer beamwidth, were therefore constructed. The simplest is a slab galaxy, modeled by a cosecant distribution in galactic latitude which levels off five degrees from the plane. Since dust and neutral bydrogen are correlated, a better estimate of the dust distribution may be given by surveys of neutral hydrogen emission at $21 \mathrm{~cm}$ wavelength. Two surveys covering the northern shy (Heiles and Habing, 1974; Weaver and Williams, 1973) were integrated over the velocity-dispersed line profiles and convolved witb the radiometer beam to form the second galactic model, which is shown in celestial coordinates in Figure 5.3.

Fits including galactic models are given in Table 5.7. The fitted "galaxy model" coefficient in Table 5.7 represents galactic emission averaged over the region within five degrees of the galactic plane, in mK. Galaxy model 1, the trancated cosecant law, has maximum and average values within five degrees of the plane of 11.5 with respect to its value at the pole. Model 2 , the convolved neutral hydrogen map, reaches a maximum value of 34 times its polar value at 
Table 5.5. Dipole-only fits to individual tights and to combined flights in mK antenne temperature. Data within tve degrees of the galactic plane has been excluded, and errors are statistical only.

\section{November Flight}

\begin{tabular}{|c|r|ccc|}
\hline Term & \multicolumn{1}{|c|}{ Magnitude } & \multicolumn{3}{|c|}{ Correlation Coeffients } \\
\hline$T_{x}$ & $-2.532 \pm .136$ & 1.000 & 0.319 & 0.553 \\
$T_{y}$ & $0.725 \pm .147$ & & 1.000 & 0.509 \\
$T_{x}$ & $-0.039 \pm .146$ & & & 1.000 \\
\hline \multicolumn{3}{|c|}{$\chi^{2}=11465 / 11296$} \\
\hline
\end{tabular}

April Flight

\begin{tabular}{|c|c|crr|}
\hline Term & Magnitude & \multicolumn{3}{|c|}{ Correlation Coefleients } \\
\hline$T_{x}$ & $-2.833 \pm .114$ & 1.000 & 0.220 & $\begin{array}{r}-0.369 \\
-0.379 \\
T_{y}\end{array}$ \\
$T_{z}$ & $\begin{array}{r}0.514 \pm .113 \\
-0.310 \pm .110\end{array}$ & & 1.000 & 1.000 \\
\hline \multicolumn{3}{|c|}{$\chi^{2}=14346 / 14059$} \\
\hline
\end{tabular}

\section{Both Flights}

\begin{tabular}{|l|c|crr|}
\hline Term & Magnitude & \multicolumn{3}{|c|}{ Correlation Coeflcients } \\
\hline$T_{x}$ & $-2.757 \pm .077$ & 1.000 & 0.075 & 0.042 \\
$T_{y}$ & $0.545 \pm .080$ & & 1.000 & $-\begin{array}{r}0.009 \\
1.000\end{array}$ \\
$T_{z}$ & $-0.283 \pm .073$ & & & \\
\hline \multicolumn{3}{|c|}{$\chi^{2}=25816 / 25363$} \\
\hline
\end{tabular}




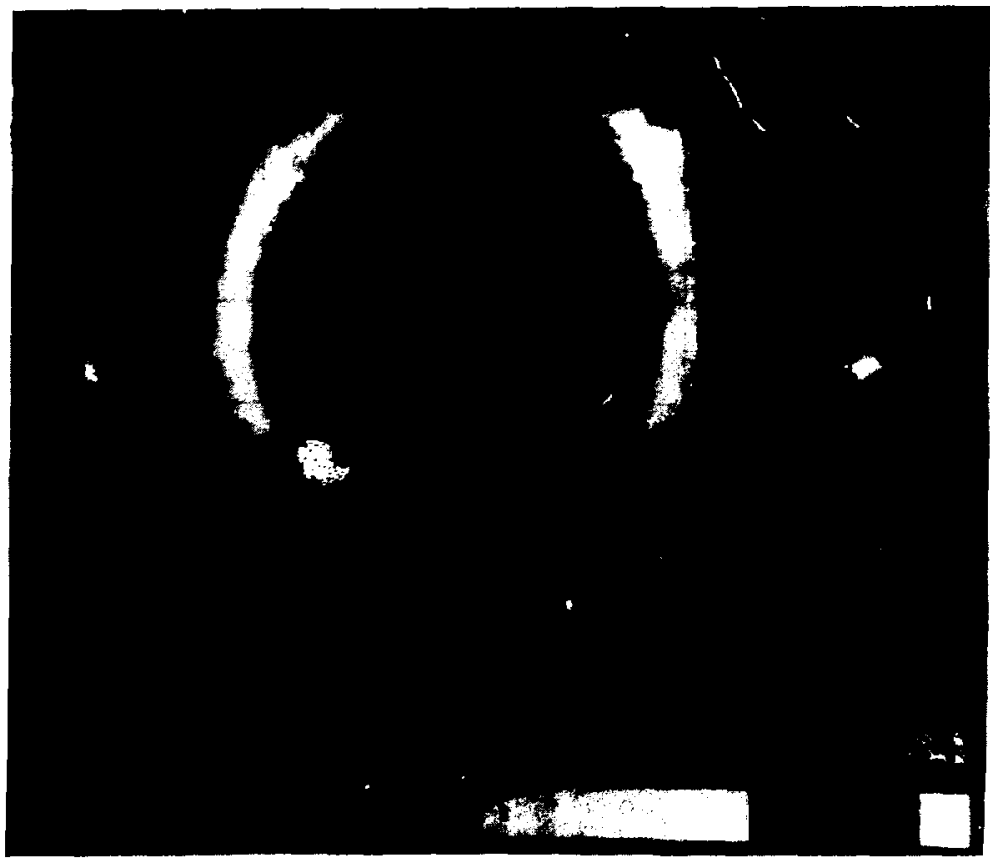

ХВВ $830-10234$

Figure 5.3. Sine equal-al a projection of 21-cu HI emiscion in 1 he northers sky, used for galasy model 2. Map coordinate are $1 \mathrm{ho}$ sanc as for Figure 4.1. Cnits are proportional to integr $1 t e d 21$-ein line strength averaged over a frecuewry range which includes mo: galactic JIJ emisciuj. Data art' from Hejles and Tlabing. (19i3) and Weares and Willams. (19it) 
Table 5.6. Effects of galactic cuts on dipole-plus-quadropole fits, in mK antenna temperature. All errors are statistical only.

\begin{tabular}{|c|r|r|r|}
\hline Term & No Galactic Cut & Cut $|b|<5^{\circ}$ & Cut $|b|<10^{\circ}$ \\
\hline$T_{x}$ & $-2.73 \pm .08$ & $-2.76 \pm .08$ & $-2.77 \pm .09$ \\
$T_{y}$ & $0.47 \pm .08$ & $0.54 \pm .09$ & $0.56 \pm .10$ \\
$T_{z}$ & $-0.22 \pm .07$ & $-0.25 \pm .08$ & $-0.22 \pm .08$ \\
$Q_{2}$ & $0.18 \pm .10$ & $0.12 \pm .10$ & $0.14 \pm .11$ \\
$Q_{3}$ & $0.19 \pm .09$ & $0.13 \pm .10$ & $0.11 \pm .11$ \\
$Q_{4}$ & $-0.06 \pm .06$ & $-0.05 \pm .07$ & $-0.04 \pm .08$ \\
$Q_{5}$ & $0.05 \pm .06$ & $0.07 \pm .06$ & $0.05 \pm .07$ \\
\hline Number of & & & \\
Points & 29161 & 25363 & 21691 \\
\hline
\end{tabular}

one spot on the galactic plane and averages 23 times the polar intensity over the band within five degrees of the galactic equator.

Table 5.7 implies an averaged galactic emission over the region less than five degrees from the galactic plane of $0.53 \pm 0.22 \mathrm{mK}$ for model 1 and $0.50 \pm 0.21 \mathrm{mK}$ for model 2 . Neither galactic model yields a significant fit when data within five degrees of the galactic plane is excluded.

\subsection{Sky Maps}

In addition to being fitted to spherical harmonics, the data can in principle be inverted to yield a map of the sky. Formally this involves a least-squares fit of the data to coeffients representing the temperature of each pixel in the inverted map. Since the data are insensitive to the addition of a constant to all points in the map, an additional parameter such as the temperature of a given cell must be specifled to determine the solution uniquely. Furthermore, depending on the sky coverage and the interconnectedness of the data (i.e., the way in which map elements are linked to each other by measurements), other degeneracies can exist. Wi $W$ balloon 
Table 5.7. Dipole-plus-quadrupole fits, in mK atenna temperalure, with and withont galactic models. Galactic model coeficients represent emission averaged over the region within five degrees of the galactic plane. Correlation coefflcients between the galactic terms and multipole parameters are snown; coefficients among the multipole parameters are similar to those given in Table 5.2. No galactic cuts were made, and all errors are statistical.

\begin{tabular}{|c|c|c|c|c|c|}
\hline & Fits & & & $\begin{array}{r}\text { Galasy } \\
\text { Correlation }\end{array}$ & $\begin{array}{l}\text { Term } \\
\text { Coefflcients }\end{array}$ \\
\hline Term & $\begin{array}{c}\text { No } \\
\text { Galaxy Model }\end{array}$ & Model 1 & Model 2 & Model $]$ & Model 2 \\
\hline $\begin{array}{l}T_{x} \\
T_{y} \\
T_{z} \\
Q_{2} \\
Q_{3} \\
Q_{4} \\
Q_{5}\end{array}$ & $\begin{array}{r}-2.73 \pm .08 \\
0.47 \pm .08 \\
-0.22 \pm .07 \\
0.18 \pm .10 \\
0.19 \pm .09 \\
-0.06 \pm .06 \\
0.05 \pm .06\end{array}$ & $\begin{array}{r}-2.72 \pm .08 \\
0.49 \pm .08 \\
-0.25 \pm .07 \\
0.06 \pm .11 \\
0.17 \pm .09 \\
0.03 \pm .08 \\
0.11 \pm .06\end{array}$ & $\begin{array}{r}-2.72 \pm .08 \\
0.50 \pm .08 \\
-0.25 \pm .07 \\
0.05 \pm .11 \\
0.16 \pm .09 \\
0.03 \pm .07 \\
0.10 \pm .06\end{array}$ & $\begin{array}{r}.05 \\
.10 \\
-.19 \\
-.47 \\
-.09 \\
.54 \\
.39\end{array}$ & $\begin{array}{r}.04 \\
.12 \\
-.17 \\
-.50 \\
-.11 \\
.49 \\
.28\end{array}$ \\
\hline $\begin{array}{c}\text { Galaxy } \\
\text { Model } 1 \\
\text { (mK, equatorial) }\end{array}$ & - & $0.53 \pm .22$ & - & 1.00 & - \\
\hline $\begin{array}{c}\text { Galasy } \\
\text { Model } 2 \\
\text { (mK, equatorial) }\end{array}$ & - & - & $0.50 \pm .21$ & - & 1.00 \\
\hline$\chi^{2}$ & 29760.39 & 29754.78 & 29754.15 & & \\
\hline DOF & 29153 & 29152 & 29152 & & \\
\hline
\end{tabular}


data taken at a single latitude, the radiometer compares the sky at one declination with exactly one other declination. An intensity distribution independent of right ascension and symmetric in declination about the midpoint between the two beams will not be seen in, or recoverable from, differential measurements.

Making a sky map with 3 degree by 5 degree pirels from this data set is equivalent to inverting a 2269 by 2269 matrix whose non-diagonal terms represent the differential measurements connecting different pirels. Fortunately for the analysis but unfortunately for the quality of the end result, very few (20000) of the over flve million cerms are non-zero. Details of the iterative Gauss-Seidel inversion used to create a map are given in Appendix E. The iterations converged, but since the matrix is poorly conditioned, it is not clear how well the solution represents the original intensity distribution.

A reconstructed sly map from the flight data is shown in Figure 5.4. Spherical harmonic flts to the map qualitatively recover the coeffieients found in direct fits to the data for both flight. data and Monte Carlo fight simulations. However, the quantitative agreement between direct. fits and fits to reconstructed maps is poor, with there being only a $1 \%$ chance that the fits represent the same data set when considered as independent measurements. Furthermore, the inversicn procedure introduces noise. In maps reconstructed from flight data, the chi-square for spherical barmonic fits to the map increased with the number of iterations performed in inverting the map. Comparison of single and double precision inversion routines showed that n'merical error was not responsible for the increase. The same increase in noise was observed in fits to maps inverted from Morte Carlo flight simulations.

\subsection{Systematic Error Analysis}

Errors quoted in the flts earlier in this chapter represent only statistical uncertainty resulting from radiometer noise. Two kinds of systematic errors must giso be estimated - those 


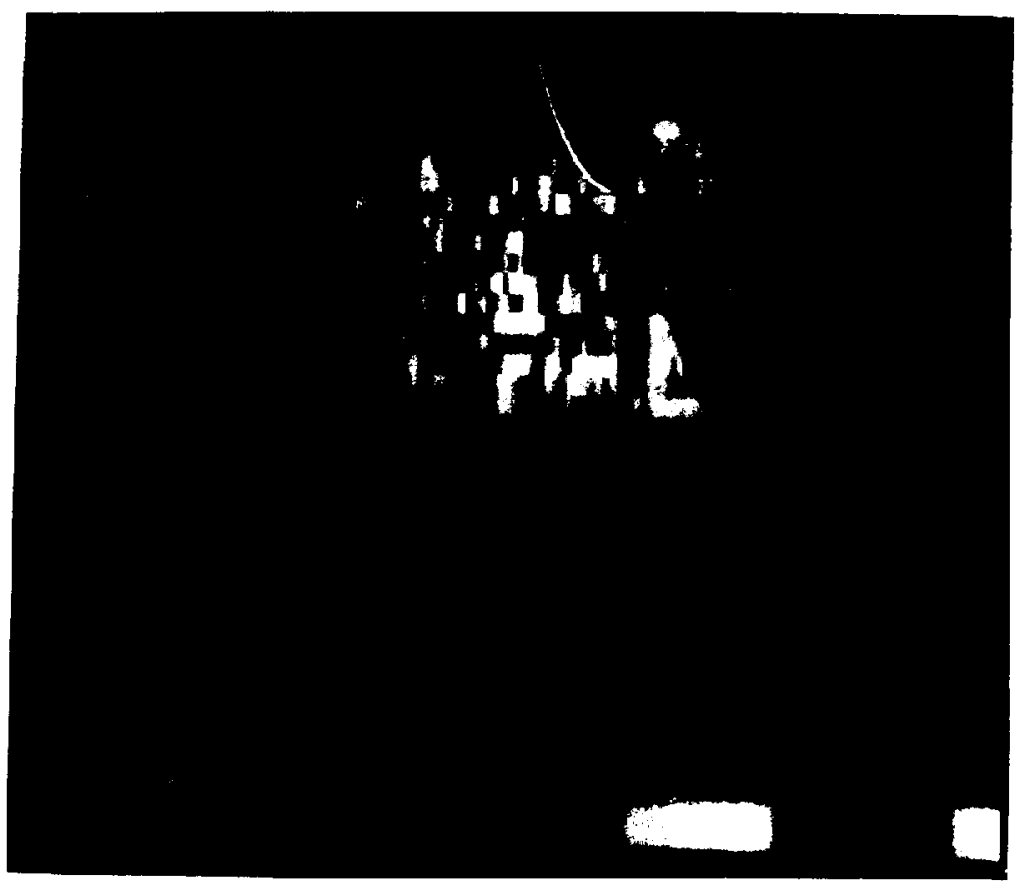

XBB $8311-10235$

Figure 5.4. Reconstructed $90 \mathrm{GHz}$ sly mapas a sine equal-area projection in the same coordinates as Figures 4.1 and 5.3. Scale runs from -8 to $8 \mathrm{ml}$. 
such as atmospheric or galactic emission which can generate spurious signals, and those such as pointing errors which can have the effect of generating quadrupole terms from the existing dipole anisotropy.

\subsubsection{Errors of the First Kind}

\subsubsection{Galactic Sources}

At centimeter and longer wavelengths, galactic emission is primarily synchrotron radiction (antenna temperature $T_{a} \sim \nu^{-2.8}$ ) and thermal brehmsstrahlung emission $\left(T_{a} \sim \nu^{-2.1}\right)$. Bounds on these contributions are obtained by scaling lower trequency surveys. Scaling the Haslam et al. (1982) $408 \mathrm{MHz}$ galactic survey, which is dominated by synchrotron radiation, limits the maximum synchrotron contribution anywhere in the sky at $90 \mathrm{GBz}$ to under $0.1 \mathrm{mK}$ when convolved over the radiometer beamwidih.

Data on thermal emission from ionized hydrogen regions has been collected, convolved with a 7 degree beam, and referred to $33 \mathrm{GHz}$ by Witebsky (1978). Scaling thase data to 90 $\mathrm{GHz}$ with a temperature spectral index of -2.1 gives a masimum intensity of $0.5 \mathrm{mK}$ on the galactic plane for the Cygnus arm of the galaxy. The maximum value more than five degrees off the galactic plane is about $0.1 \mathrm{mK}$.

At millimeter wavelengths and shorter, dust emission is the primary galactic contaminant. The spectral and spatial distribution of dust emission is poorly known, and observations made at other frequencies cannot. be scaled to $90 \mathrm{GHz}\left(3 \mathrm{~cm}^{-1}\right)$ as easily for dust as for thermal and synchrotron radiation. Owens et al. (1979) measured dust emission in the galactic plane in the 10 to $25 \mathrm{~cm}^{-1}$ band. For a source having an emissivity proportional to frequency squared over that band, their observed flux corresponds to antenna temperatures at $22 \mathrm{~cm}^{-1}$ avetaging about $28 \mathrm{ml}$ : on the galactic plane between 0 and 45 degrees galactic longitude. Only 
one source, haring a peak antenna temperature of $15 \mathrm{mK}$, was found more than five degrees of the plane. Owens et al. state that their "best guess" for scaling observations to different frequencies is to take the emissivity to be proportional to frequency squared.

The best experimental limits to the intensity of dust emission at $90 \mathrm{GHz}$ are the fits to galactic models in this thesis. These show the galactic emission less than five degrees from the galactic plane to be on the order of $0.5 \mathrm{mK}$. Any diffuse thermal emission, which was not incladed in the compilation by Witebsky (1978), is included in this limit.

Possible effects of diffuse of-plane galactic emission on multipole fits were investigated by generating simulated observations of an extended source in the region of the off-plane emission reported by Owens et al. The worst-case source intensity is given by scaling the $15 \mathrm{mK}$ Owens et al. intensity to $90 \mathrm{GHz}\left(3 \mathrm{~cm}^{-1}\right)$ using a temperature spectral index of 1.5 . The center frequency of the Orens et al. apparatus for a source with that spectral index is $16.7 \mathrm{~cm}^{-1}$ (S. Meyer, private communication). Scaling from $16.7 \mathrm{~cm}^{-1}$ with a temperature spectral indes of 1.5 gives a source intensity at $3 \mathrm{~cm}^{-1}$ of $1.1 \mathrm{mK}$. Such a source would contribute $+70 \mu \mathrm{K}$ to $Q_{2}$ and would change the other dipole and quadrupole terms by less than $50 \mu \mathrm{K}$.

\subsubsection{Interplanetary Suurces}

At still shorter wavelengths (10 to 100 micron), the dominant source of difuse emission is likely to be interplanetary dost particles (zodiacal light). Zodjacal emission at wavelengths longer than its peak at about 10 microns is not well characterized. Measurements are not inconsistent with a $300 \mathrm{~K}$ spectrum having an emissivity proportional to frequency and an optical depth at 10 microns of about $5 \times 10^{-8}$ (NASA, 1977). This spectral dependence gives a zodiacal contribution of tens on nanokelvins at $90 \mathrm{GHz}$. 


\subsubsection{Ionospheric Free-Free Emission}

A more local source of ertraneous radiation is the residual atmosphere above the balloon. Corey (1978) has calculated a firm upper limit of $0.6 \mathrm{mK}$ on ionospheric free-free emission at 19 GHz with a probable value signiflcantly less than that. The antenna temperaure of thermal freefree emission is inversely proportiona to frequency squared, so the corresponding upper bound at $90 \mathrm{GH} z$ is a negligible $0.03 \mathrm{mK}$.

\subsubsection{Atmospheric Molecular Emission}

Molecular emission from the atmosphere can be estimated from the emission line parameters of atmospheric constituents, together with a description of atmospheric physical parameters as a function of altitnde. The Air Force Cambridge Geophysical Laboratory has compiled line parameters from the radio to the optical (referenced in Weiss, 1980), and the $1962 \mathrm{U}$. S. Standard Atmosphere (Cole, 1965; tabulated in Ulaby et al., 1981) describes atmospheric physical properties. Calculated zenith atmospheric emission at $30 \mathrm{~km}$ is shown in Figure 2.2 for frequencies less than $150 \mathrm{GHz}$. At $90 \mathrm{GHz}$, the dominant contribution is $5 \mathrm{mK}$ from the pressure-broadened wings of oxygen magnetic dipole transitions centered at 60 and $120 \mathrm{GHz}$. Wings of rotational water lines, the nearest of which are at 22 and $183 \mathrm{GHz}$, add another $0.2 \mathrm{mK}$. The ozone spectrum is full of rotational lines in the microwave region, but the $90 \mathrm{GHz}$ radiometer $\mathrm{RF}$ passband between 88.4 and $91.6 \mathrm{GHz}$ does not include any major ones and at $30 \mathrm{~km}$ picks up about $0.1 \mathrm{mK}$ from ozone line wings. Both the zenith column density and the line widths increase with pressure, so off-peak atmospheric emission grows faster than linearly with pressure. At 27.5 $\mathrm{km}$, the oxygen emission is $15 \mathrm{mK}$ and it increases to $40 \mathrm{mK}$ at $25 \mathrm{~km}$.

For a layered slab atmosphere with zenith temperature $T_{0}$, the emission at zenith angle $\theta$ is given by 


$$
T(\theta)=T_{0} \sec \theta
$$

$A$ gondola tilt aiggle of $\Delta \theta$ generates a difference between the beam zenith angles of $2 \Delta \theta$ and a differential atmospheric signal of

$$
T(\theta, \Delta \theta)=2 T_{0} \sec \theta \tan \theta \Delta \theta
$$

For radiometer beam zenith angles of 45 degrees at $27.5 \mathrm{~km}$ altitude, the $\mathbf{a}^{\mathrm{t}}$ mospheric signal is $23 \mathrm{mK}$ and the differential atmospheric signal is $0.8 \mathrm{mK}$ per degree radiometer tilt.

\subsubsection{Gronnd Pickup}

The earth has a radiometric temperature on the order of $300 \mathrm{~K}$ at $90 \mathrm{GHz}$. The antenna sidelobe pattern, togethe) with the groundshiplds, must therefore provide $65 \mathrm{db}$ of rejection to ensure that radiation from the ground contributes less than $0.1 \mathrm{mK}$. Since it was not possible to measure the far-sidelobe radiometer response after the gondola was lost, we instead calculate an estimate of the power diffracted over the groundshield.

The groundshield is modeled by a cylindricai cone having an opening angle equal to that of the actual hexagonal shield, with a radius equal to that of the hexagon's inscribed circle. For tbe calculation, the horn aperture is take's to be in the center of the cone, $12.7 \mathrm{~cm}$ below the plane of the cone's edge, aud pointing upwards at a 45 degree angle. (The real horn pas at the same depth and angle but was sei back $4.3 \mathrm{~cm}$ behind the chopper wheel, which was at the certer of the shield.)

At each point on the edge of the shield, the far-field antenna pattern is used to determine the antenna gain. However, with the far-fleld approximation strictly valid for regions farther ihan $\pi a^{2} / \lambda \approx 60 \mathrm{~cm}$, with $a$ the horn radius, using far-fleld patterns for the groundshield edge $33 \mathrm{~cm}$ away is not strictly justifled. Radiation from each region on the ground is assumed to difract. 
over only that section of the groundshield edge directly between it and the horn. Fresnel theory for diffraction over a conducting straightedge is used to compute the ratio of the power diffracted into the horn to the power incident (Born and Wolf, 1964, p. 578). The diffracted power over each portion of the edge, multiplied by the appropriate antenna gain, is integrated around the groundshield to give the total diffracted contribution.

The intensity of radiation diffracted over a conducting edge is polarization-dependent. In the polarization accepted by the horn, E field parailel to the shield edge, the interior shield diffracts an estimated $0.03 \mathrm{mK}$ into the horn. Diffracted power in the rejected mode is $0.98 \mathrm{mK}$.

The accuracy of this calculation, in light of the assumptions made, is certainly not high. However, several factors indicate that ground contamination may be less than that estimated here. On all flights, there was shielding in addition to the interior hexagonal shield. In November, 1981, panels extending the hexagonal groundshield horizontally for $13 \mathrm{~cm}$ were attached to all six sides, requiring ground radiation to diffract around two edges to reach the horn. On the April, 1982 flight, a second groundshield completely surrounded the gondola; the tops of the two shields were at the same height which again required ground radiation to difract twice in order be detected. Furthermore, only the anisotropy in diffacted ground radiation can contaminate CBR anisotropy data. Although the radiometric temperature of seawater is on the order of $100 \mathrm{~K}$ less than that of land (Ulaby et al., 1981), atmospheric emission reflected off the water and also added between the ground and the balloon minimize the observed temperature difference between land and water. Since power from near the horizon dominates the diffacted contribution, the atmospheric path lengths are long enough for their optical depth to become appreciable. If the maximum antenna temperature difference from horizon to horizon is $30^{\circ} \mathrm{C}$, the effect of anisotropic ground emission is an order of magnitude less than the total power diffracted into the horn. 


\subsubsection{Balloon Contributions}

The balloos emits thermal radiation and also reflects power from the ground. Botb contributions are negligibly small. Tbe absorption coefilcient of polyethylene was measured to be $1.5 \times 10^{-2} \mathrm{~cm}^{-1}$ at $90 \mathrm{GHz}$. The balloon thickness $t$ is $0.5 \mathrm{mil}=1.3 \times 10^{-3} \mathrm{~cm}$. Areraging the $4 \pi r^{2} t$ volume of the polyethylene membrane over its projected ar: : of $\pi r^{2}$ gives an average projected thickness of 4 t or $5.2 \times 10^{-3} \mathrm{~cm}$, for a mean optical depth of : $3 \times 10^{-5}$. At a fiysical temperature of $300 \mathrm{~K}$, the balloon would have a brightness temperature c $23 \mathrm{mK}$.

The reflection coefficient for a thin dielectric film of thickness $t$ and dielectric constant $n$ at normal jncidence is

$$
R=\left(\frac{4 \pi n t}{\lambda} \frac{n-1}{n+1}\right)^{2}
$$

For a $1.3 \times 10^{-3} \mathrm{~cm}$ fllm of polyethylene $(n \approx 1.5)$ at $\lambda=0.33 \mathrm{~cm}, R=2.1 ;: 10^{-4}$. Reflected esrth power will have a brightness temperature of $R \times 300 \mathrm{~K}$ or $62 \mathrm{mK}$. Although the reflection coefilient increases as the anglt of incidence increases, reflection paths having incidence angles greater than 50 degrees do not intercept the earth and need not be considered.

Summing the two contributions gives the balloon a brightness temperature of $87 \mathrm{mK}$ when viewed head-on. Since all points on the balloon are at least 29 degrees of the antenna axis, the antenna gain over the balloon is less than $3.0 \times 10^{-5}$. Using this bound as an upper limit and integrating over the balloon gives a maxisum emitted plus reflected contribution to the radiometer sigual of $23 \mu \mathrm{K}$.

\subsubsection{Synchronous System Madulations}

Modulations in system parameters (gain, offet) synchronous with gondola rotation will be interpreted as a spurious anisotropy signal. As discussed in Chapter 3 , signals generated by rotation within the earth's magnetic field are under $0.1 \mathrm{mK}$; variations in the chopper wheel's 
physical temperature bounded in Chapter 4 induce a sigal less than $0.03 \mathrm{mK}$. Variations of the second (non-cryogenic) IF amplifler temperature synchronous with balloon rotation were found to be less than $0.025^{\circ} \mathrm{C}$ during flight. With the temperature dependence of the second IF gain measured to be less than $0.5 \%$ per degree $C$, temperature-induced gain variations acting on the $200 \mathrm{mK}$ offset generate a spurious racironous signal of less than $30 \mu \mathrm{K}$.

5.3.2 Errors of the Secold Kind

\section{3.i.1 Calibration Uncertainty}

Determination of the system calibration constant, known to $\pm 6 \%$, is discussed in Appendix C. The calibration was found to be the same throughout a flight ( to 1\%) and from one flight to another (to $3 \%$ ). Unmodeled variation in the system calibration from flight to flight or within a flight would be a source of error which would have the effect of taking part of the dipole anisotropy and attributing it to higher order moments. These errors were bounded by sensitivity studies in which the system gain was assumed to vary within or between fights. The consequent. changes in the values for fitted multipole parameters indicate possible effects of calibration errors. Varying the relative November to April calibration by $\pm 3 \%$ changes the fitted value of $T_{x}$ by 40 $\mu \mathrm{K}, T_{y}$ and $T_{z}$ by less than $20 \mu \mathrm{K}$, the quadrupole parameters $Q_{2}$ and $Q_{3}$ by less than $10 \mu \mathrm{K}$, and $Q_{4}$ and $Q_{5}$ by less than $20 \mu \mathrm{K}$.

The error in the absolute calibration oi $6 \%$ cannot put dipole power into the quadrupole terms. Instead, it introduces an ancertainty in each coefficient proportional to the coefficient's magnitude and is significant only for $T_{x}$, where the $170 \mu \mathrm{K}$ absolute calibration uncertainty is the dominant error. 


\subsubsection{Pointing Errors}

Errors in determining the gondola orientation can also redirect the dipole anjsotropy into other dipole or higher order terms. Moon and sun observatjons in Teras (November, 1981) and in Brazil (November, 1982) indicate that the aximuth angle of the radiometer beams is know to \pm 1.5 degrees. Total error in the radiometer beam positions is taken to be less than \pm 2 degrees.

Sensitivity studies were done in which the caiculated azimuth angle of the gondola was skewed by \pm 2 degrees for the Navember and April flights. Since diferent magnetometers were used for the two flights, the erfors in azimuth for November and for April are not necessarily correlated, so the results were added in quadrature. The total change in the $T_{x}$ component was less than $20 \mu \mathrm{K}, T_{y}$ less than $70 \mu \mathrm{K}, T_{z}$ less than $50 \mu \mathrm{K}, Q_{2}$ asd $Q_{3}$ less than $40 \mu \mathrm{K}$, and $Q_{4}$ and $Q_{5}$ less than $30 \mu \mathrm{K}$.

\subsection{Summary and Conversion to Thermodynamic Temperature}

The breakdown of the total estimated errors on the multipole parameters given in Table 5.2 are shown in Table 5.8. The specified contributions itemized in Section 5.3 are listed along with the statistical errors, and all components are summed in quadrature to give the totrl error in microKelvins.

The dipole and quadrupole anisotropy coeficients, with total errors, are given in Table 5.9 both in antenna temperature and in thermodynamic temperature corresponding to a $2.7 \mathrm{~K} \mathrm{CBR}$ temperature. The conversion from antenna to thermodynamic temperature is discussed more fully in the following chapter. 
Table 5.8. Contributions to total error of dipole and quadrupole parameters, in microKelvins.

\begin{tabular}{|c|c|c|c|c|c|}
\hline Term & $\begin{array}{c}\text { Statistical } \\
\text { Error }\end{array}$ & $\begin{array}{c}\text { Absolute } \\
\text { Calibration }\end{array}$ & $\begin{array}{c}\text { Relative } \\
\text { Calibration }\end{array}$ & $\begin{array}{c}\text { Pointing } \\
\text { Error }\end{array}$ & $\begin{array}{c}\text { Total } \\
\text { Error }\end{array}$ \\
\hline$T_{x}$ & 80 & 170 & 40 & 20 & 190 \\
$T_{y}$ & 90 & 30 & 20 & 70 & 120 \\
$T_{z}$ & 80 & 20 & 20 & 50 & 100 \\
$Q_{2}$ & 100 & 10 & 10 & 40 & 110 \\
$Q_{3}$ & 100 & 10 & 10 & 40 & 110 \\
$Q_{4}$ & 70 & 0 & 20 & 30 & 80 \\
$Q_{5}$ & 60 & 0 & 20 & 30 & 70 \\
\hline
\end{tabular}

Table 5.9. Dipole-plus-quadrupole anisotropy at $90 \mathrm{GHz}$ in antenna temperature and in thermodynamic temperature. Error estimates include systematic errors.

\begin{tabular}{|l|r|r|}
\hline Term & Antenna Temperature & $\begin{array}{r}\text { Thermodynamic Temperature } \\
\text { (for } T_{\mathrm{CBR}}=2.7 \mathrm{~K} \text { ) }\end{array}$ \\
\hline$T_{\mathbf{I}}$ & $-2.76 \pm 0.19$ & $-3.40 \pm 0.23$ \\
$T_{\mathbf{y}}$ & $0.54 \pm 0.12$ & $0.67 \pm 0.15$ \\
$T_{\mathbf{z}}$ & $-0.25 \pm 0.10$ & $-0.31 \pm 0.12$ \\
$Q_{2}$ & $0.12 \pm 0.11$ & $0.15 \pm 0.14$ \\
$Q_{3}$ & $0.13 \pm 0.11$ & $0.16 \pm 0.14$ \\
$Q_{4}$ & $-0.05 \pm 0.08$ & $-0.06 \pm 0.10$ \\
$Q_{5}$ & $0.07 \pm 0.07$ & $0.09 \pm 0.09$ \\
\hline
\end{tabular}




\section{Chapter 6}

\section{Interpretation}

\subsection{Dipole Anisotropy}

The dipole anssotropy measured by this experiment is presented with total errors in Table 6.1. Table 6.2 repeats these results in spherical coordinates and also includes results of the most recent publications by the Princeton and the Berkeley groups. Not included in Table 6.2 is Lubin et ah, (1983a), the initial publication of the results reported in this thesis. Those injtial results differ from the ones reported here primarily in a $6 \%(1 \sigma)$ adjustment of the value used for the system calibration. The calculation of system gain used in the initial results included a term based on the in-flight pop-up calibrator observations which jncreased slightly the calibration $(\mathrm{mK} /$ volt ) at altitude relative to that on tbe ground. Further analysis has show $n$ that the pop-up calibrator observations on the ground were not sufficiently well-determined to justify the increase, and no such correction has been applied in this work. See Appendix C.

\subsubsection{Direction}

The direction of the dipsle measured by this experiment agrees with that of the others in Table 6.2 except for a statistically significant discrepancy in declination with respect. to Smoot 
Table 6.1. $90 \mathrm{GHz}$ dipole-only anisotropy in antenna temperature and in thermodynamic temperature for $T_{\text {CBR }}=2.7 \mathrm{~K}$. Data within five degrees of the galactic plane has been excluded, and error estimates include systematic errors.

\begin{tabular}{|c|c|c|}
\hline Term & Antenna Temperature & $\begin{array}{c}\text { Thermodynamic Temperature } \\
\text { (for } T_{\mathrm{CBR}}=2.7 \mathrm{~K} \text { ) }\end{array}$ \\
\hline$T_{x}$ & $-\because .76 \pm 0.19$ & $-3.40 \pm 0.23$ \\
$T_{y}$ & $0.54 \pm 0.11$ & $0.67 \pm 0.14$ \\
$T_{z}$ & $-0.28 \pm 0.09$ & $-0.35 \pm 0.11$ \\
\hline
\end{tabular}

and Lubin (1979). In the comparisons, dipole-only flts are quoted for experiments not claiming significant quadrupole terms. The dipole-only fit should equal the dipole part of a dipole-plusquadrupole fit if there is no significant quadrupole power and if there are small correlations between dipole and quadrupole terms. A comparison of Tables 5.9 and 6.1 shows that the dipoleonly and the dipole-plus-quadrupole fits agree quite well for this experiment. Although Fixsen et al (1983) found no evidence for a quadrupole, their $T_{z}$ and $Q_{1}$ components are rather highly correlated (correlation coeffient $=-0.70$ ). The dipole result quoted in Table 6.2 for Fixsen et al. has been reconstructed from their dipole-plus-quadrupole fit by inverting the solution vector and error matrix to recover their original data vector and moment matrix, and solving again for only the dipole terms. The Fixsen et al. dipole-only result in Table 6.2 has a declination 3 degrees more positive and a right ascension 1 degree greater than their dipole-plus-quadrupole fit. The Fixsen et al. dipole direction agrees very mell with this measurement.

Comparison of this experiment to Boughn et al. (1981) is complicated by the quadrupole anisotropy claimed by Boughn et al. If the quadrupole were real, then dipole-only fits with incomplete sky coverage would be corrupted by quadrupole power and would not represent the true dipole anisotropy. Boughn et al. give data for the dipole and four of the five quadrupole components, with $T_{z}$ (fitted) $=T_{x}$ (true) $+1.1 Q_{1}$. Their quoted dipole anisotropy, given 
Table 6.2. Comparison of dipole anisotropies measured by different experiments. Magnitude is mK thermodynamic temperature for $T_{\text {CBR }}=2.7 \mathrm{~K}$.

\begin{tabular}{|c|c|c|c|c|c|}
\hline Reference & $\begin{array}{c}\text { Frequency } \\
\qquad(\mathrm{GHz})\end{array}$ & $\begin{array}{l}\text { Magnitude } \\
\text { (mK) }\end{array}$ & $\begin{array}{c}\text { Right } \\
\text { (degrees) }\end{array}$ & $\begin{array}{c}\text { Ascension } \\
\text { (hours) }\end{array}$ & $\begin{array}{c}\text { Declination } \\
\text { (degrees) }\end{array}$ \\
\hline This work & 90 & $3.48 \pm 0.24$ & $168.8 \pm 1.9$ & $11.3 \pm 0.1$ & $-5.7 \pm 1.8$ \\
\hline $\begin{array}{l}\text { Fixsen et al., } \\
1983\end{array}$ & 24.5 & $3.12 \pm 0.18$ & $169.2 \pm 1.6$ & $11.3 \pm 0.1$ & $-5.3 \pm 1.6$ \\
\hline $\begin{array}{l}\text { Smoot and Lubin, } \\
1979\end{array}$ & 33 & $3.1 \pm 0.4$ & $172 \pm 6$ & $11.5 \pm 0.4$ & $9.6 \pm 6$ \\
\hline $\begin{array}{l}\text { Boughn et al., } \\
1981\end{array}$ & $\begin{array}{l}19,24.8 \\
31.4,46\end{array}$ & $3.78 \pm 0.30$ & $174 \pm 3$ & $11.6 \pm 0.2$ & $-12 \pm 5$ \\
\hline
\end{tabular}

${ }^{1}$ Dipole-only fit reconstructed according to Section 6.1.1. Table 1.1 entry is dipole portion of combined dipole-plus-quadrupole fit.

${ }^{2}$ Dipole portion of combined dipole-plus-quadrupole fit.

in Table 6.2, is the dipole part of the combined dipole-plus-quadrupole fit, with the fitted $T_{z}$ converted to true $T_{z}$ by removing the $Q_{1}$ term of $0.38 \pm 0.26$ reported by Smoot aE $a^{2}$ Lubin (1979). If the same dipole-only reduction used on Fixsen et al. is applied to Boughn et al., the dipole declination becomes $-6 \pm 5$ degrees and the right ascension decreases to 171 degrees $(11.4$ hours), which is in better agreement with this experiment. The magnitude of the reconstructed Boughn et al. dipole-only fit is $3.74 \pm 0.30 \mathrm{mK}$.

\subsubsection{Magnitude}

The magnitudes of the dipole results are qualitatively in agreement. They are reported in Table 6.2 as thermodynamic temperature anisotropies assuming a CBR $2.7 \mathrm{~K}$ blackbody spectrum. A simple weighted average of their amplitudes is $3.33 \pm 0.12 \mathrm{mK}$. The $x^{2}$ value of the entries with respect to this average is 4.38 for 3 DOF, with over half coining from the Boughn et al. measurement. The confidence level is $22 \%$. In order to account for the direction as well in the sverage, a quantitative analysis would need tc generate an effective total moment matrix 
and data vector for the four experiments, solve the combined system, and compute the total $\chi^{2}$ for consistently-defined solntions (e.g. all dipole-only fits). This procedure is strictly valid for experiments dominated by statistical error, which is not the case for the ones here. Since the dipole directions are in agreement, with the largest discrepancy being in the least-heavily weighted measurement, averaging the magnitudes is an adequate indication of th 5 consistency of the experiments under the assumption of a $2.7 \mathrm{~K}$ blackbody spectrum.

Since the magnitude of a motion-indnced antenna temperature anisotropy depends upon the CBR sjectral shape as well as its intensity, it is necessary to model the CBR spectrum in order to quantitatively compare the results. Coniersely, by assuming that the antenna temperature anisotropies measured at different fequencies represent the same thermodynamic anisotropy, information about the CBR spect;um can be obtained (Danese and De Zotti, 1981; Lubin et al., 1983c).

For an arbitrary CBR spectru $m$, the anisotropy at ! $\quad \cdots \nu$ is given in terms of the velocity $\beta$, the intensity spectral index $\alpha$, and the CBR antenna temperature $T_{a}$ by substituting equation (1.5) into equation (1.4b):

$$
\Delta T_{a}=(2-\alpha) \beta T_{a}
$$

For a blackbody CBR with frequency-independent thermodynamic anisotropy $\Delta T$, the parameters $\alpha$ and $T_{a}$ are related, so anisotropy measurements at different frequencies unambiguously scale according to equation $(1,0 b)$ :

$$
\Delta T_{a}(\nu)=\frac{x^{2} e^{x}}{\left(e^{x}-1\right)^{2}} \Delta T, \quad \text { Fith } x=\frac{h \nu}{k T} .
$$

\subsubsection{CBR Spectral Distortion}

Distortions from a CBR blackbody spectrum will indure corresponding departures from (1.6b). In particular, near $90 \mathrm{GHz}$ the measurement of Woody and Richards (1979) suggests 
a higher temperature and a flatter spectral index than those for blackbody spectra. For the Woody-Richards data at $90 \mathrm{GHz}, T$ is $3.18 \pm_{0.10}^{0.25} \mathrm{~K}$ with corresponding antenna temperature $T_{a} \simeq 1.47 \pm 0.2 \mathrm{~K}$. The spectral index is $\simeq 0.88$, but values between 0.85 and 1.15 cannot be ruled out. At $90 \mathrm{GHz}$, a $2.7 \mathrm{~K}$ blackbody has $T_{\mathrm{a}}=1.09$ and $\alpha=1.00$; a $3 \mathrm{~K}$ blackbody has $T_{a}=1.34$ and $a=1.11$. Both the flux and the spectral slope of the Woody-Richards spectrum would enhance the $90 \mathrm{GHz}$ anisotropy relative to what would be expected by scaling lower frequency observations using (1.6b).

The consistency between this experiment and lower frequency observations can be examined with respect to different models for the CBR spectra, paralleling the analysis of Lubin et al., (1983c). Given the CBR antenna temperature $T_{a}$ and temperature spectral index $\alpha$ at frequency $\nu$, the velocity vector $\vec{\beta}_{i}$ corresponding to each dipole anisotropy observation $\Delta \vec{T}_{i}$ can be calculated from (6.1). If the two observations $\vec{\beta}_{i}$ truly represent the same $\vec{\beta}$, then the scalar product

$$
S^{2}=\left(\vec{\beta}_{1}-\vec{\beta}_{2}\right)^{T}\left\{\mathbf{M}_{1}^{-1}+\mathbf{M}_{2}^{-1}\right\}^{-1}\left(\vec{\beta}_{1}-\vec{\beta}_{2}\right)
$$

where the $\mathbf{M}_{i}^{-1}$ are the error matrices corresponding to the $\vec{\beta}_{i}$, has a $\chi^{2}$ distribution for three degrees of freedom (Appendix D). A measure of the consistency of two measurements, then, is the probability that $\chi^{2}$ for 3 DOF would exceed the $S^{2}$ value calculated here.

Dipole magnitude measurements do not yet have precision sufficient to place very stringent limits on the CBR spectrum. Furthermore, the variation in measurements made at the same frequency suggests that systematic errors may be underestimated. Nevertheless, an analysis comparing the $90 \mathrm{GHz}$ experiment to the Fixsen et al. result at $24.5 \mathrm{GHz}$ has been carried out. Of the experiments in Table 6.2, these two are the most recent and the most precise. They are also the only ones with in-flight calibration. Tbe dipole anisotropy results for this experiment and for Fixsen et al are given in antenna temperature in Table 6.3, along with the expected anisotropies $(3-\alpha) \beta T_{\mathrm{a}}$ corresponding to different CBR models. 
Toble 6.9. Measured dipole anisotropy at 90 and $24.5 \mathrm{GHz}$, along with predicted anisotropies for different CBR spectra.

\begin{tabular}{|c|c|c|}
\hline & Th.is experiment & Fixsen et al. \\
\hline Frequency (GEz) & 90 & 24.5 \\
\hline \multicolumn{3}{|c|}{ Measired Components in $\mathbf{m K}$ antenna temperature: } \\
\hline $\begin{array}{c}\Delta T_{a, x} \\
\Delta T_{a, y} \\
\Delta T_{a, z} \\
\text { Magnitude } \\
\Delta T_{a}\end{array}$ & $\begin{array}{r}-2.76 \pm 0.19 \\
0.54 \pm 0.11 \\
-0.28 \pm 0.09 \\
2.83 \pm 0.19\end{array}$ & $\begin{array}{r}-3.01 \pm 0.17 \\
0.58 \pm 0.09 \\
-0.28 \pm 0.08 \\
3.07 \pm 0.17\end{array}$ \\
\hline \multicolumn{3}{|c|}{ Expected Magnitude $(3-\alpha) T_{a} \beta$ in $K$ antenna temperature: } \\
\hline $\begin{array}{l}2.7 \mathrm{~K} \text { Blackbody } \\
\text { 3.0 K Blackbody } \\
\text { Woody-Richards }\end{array}$ & $\begin{array}{l}2.191 \beta \\
2.531 \beta \\
3.116 \beta\end{array}$ & $\begin{array}{c}2.658 \beta \\
2.962 \beta \\
\\
\end{array}$ \\
\hline
\end{tabular}

Table 6.4 gives the $S^{2}$ values and corresponding confidence levels for the comparisons. CBR blackbody spectra with iemperatures of $2.7 \mathrm{~K}$ and $3.0 \mathrm{~K}$ are examined, as are spectra which are $2.7 \mathrm{~K}$ or $3.0 \mathrm{~K}$ blackbodies at $24.5 \mathrm{GHz}$ but are consistent with the Woody-Richards data at $90 \mathrm{GHz}$. For completeness, CBR spectra which are a $2.7 \mathrm{~K}$ blackbody at one of the two frequeusies and $3.0 \mathrm{~K}$ blackbody at the other are also examined. Table 6.4 shows that a $2.7 \mathrm{~K}$ thermal CBR spectra is quite consistent with the $24.5 \mathrm{GHz}$ and $90 \mathrm{GHz}$ anisotropy measurements. Neither the quality of the snisotropy observations, nor the precision with which the anisotropy expected from the Woody-Richards spectrum can be calculated, allows significant conclusions to be made concerning the Woody-Richards data.

For a blackbody $C B R$, the cases $T_{C B R}=2.7 \mathrm{~K}$ and $T_{C B R}=3.0 \mathrm{~K}$ can be generalized to arbitrary temperature. Figure 6.1 presents. as a function of $T_{C B R}$, the confidence levels at which 


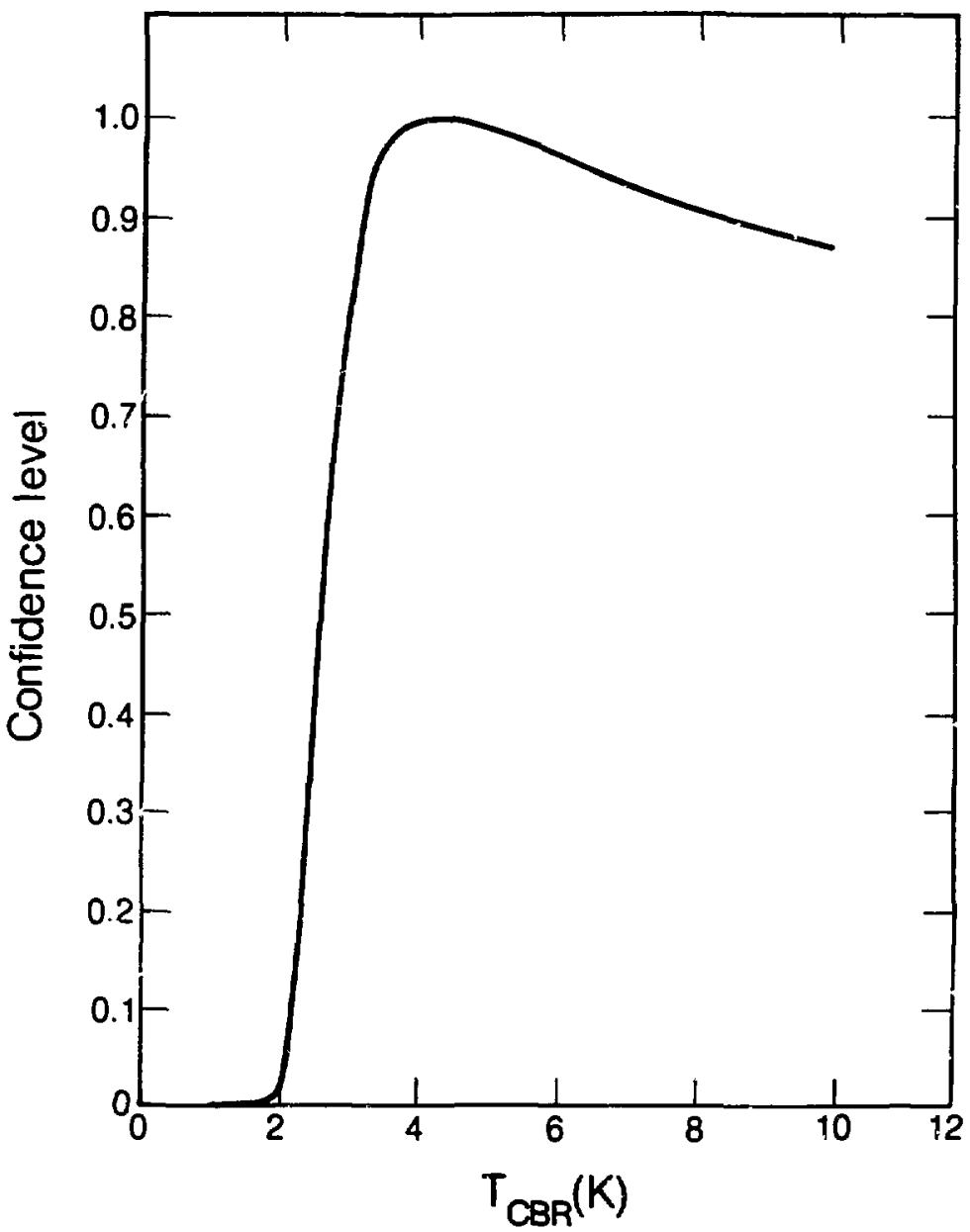

Figure 6.1. Confldence levels st which $24.5 \mathrm{GHz}$ and $90 \mathrm{GHz}$ dipole observations agree with each other sssuming a blackbody CBR, as a function of CBR temperature 
Table 6.4. Confdence levels at which anjsotropies measured at $24.5 \mathrm{GHz}$ and at $90 \mathrm{GHz}$ are consistent with each other assuming various CBR spectra.

\begin{tabular}{|c|c|c|c|}
\hline $\begin{array}{c}\text { CBR Spectrum } \\
\text { at } 24.5 \mathrm{GHz}\end{array}$ & $\begin{array}{c}\text { CBR Spectrum } \\
\text { at } 20 \mathrm{GHz}\end{array}$ & $\begin{array}{c}\text { Value of } \\
S^{2}\end{array}$ & $\begin{array}{c}\text { Confidence } \\
\text { Level }\end{array}$ \\
\hline $2.7 \mathrm{~K}$ & $3.0 \mathrm{~K}$ & 0.2027 & $97.7 \%$ \\
$3.0 \mathrm{~K}$ & $3.0 \mathrm{~K}$ & 0.9133 & $82.2 \%$ \\
$2.7 \mathrm{~K}$ & $2.7 \mathrm{~K}$ & 1.9048 & $59.2 \%$ \\
$3.0 \mathrm{~K}$ & $\mathrm{~W}-\mathrm{R}$ & 2.7451 & $43.3 \%$ \\
$3.0 \mathrm{~K}$ & $2.7 \mathrm{~K}$ & 6.9941 & $7.2 \%$ \\
$2.7 \mathrm{~K}$ & W-R & 9.0799 & $2.8 \%$ \\
\hline
\end{tabular}

the $24.5 \mathrm{GHz}$ and the $90 \mathrm{GHz}$ anisotropy measurements are consistent with each other assuming a blackbody CBR spectrum. It shows that no CBR temperature less than $2.19 \mathrm{~K}$ is consistent with the observations at bigher than a $10 \%$ confidence level, putting a $90 \%$ confldence-level minimum of $2.2 \mathrm{~K}$ on the CBR temperature. Unlike most other conclusions about the CBR spectrum derivable trom anisotropy measurements, this CBR tempersture lower limit is quite firm. Physically, a blackbody CBR spectrum cooler than the minimum would have so much less power at $90 \mathrm{GHz}$ than at lower frequencies that that the measured $90 \mathrm{GHz}$ anisotropy would imply enormous anisotropy at $24.5 \mathrm{GHz}$.

No upper bound can be placed on the CBR temperature from this analysis alone. Since the difference between the expected snisotropies at $24.5 \mathrm{GHz}$ and $90 \mathrm{GHz}$ for high temperature blackbody spectra is so small, all suffiently high temperatures are equivalent and cannot be distinguished by the two measurements. The confldence level vs. CBR temperature plot in Figure 6.1 has a very broal maximum, showing that a CBR temperature of $4.2 \mathrm{~K}$ is formally most consistent with the $24.5 \mathrm{GHz}$ and the $90 \mathrm{GHz}$ observations but also indicating that an infinitely hot CBR is consistent with the ratio of those measurements at a confldence level of $79 \%$. 


\subsubsection{Solar Velocity}

The dipole anisotropy is most readily interpreted as a measure of velocity relative to a comoving frame of reference. Since dipole anisotropy generated by other mechanisms would in general be accompanied by higher order anisotropies, the lack of quadrupole moment (see next section) supports the peculiar velocity origin of the dipole, as does the dipole's frequency independence.

Assuming a $2.7 \mathrm{~K}$ blackbody CBR, the dipole anisotropy measured by this experiment represents a solar velocity of $387 \pm 25 \mathrm{~km} / \mathrm{sec}$ towards right ascension $\alpha=11 .{ }^{h} 3 \pm 0 .{ }^{h} 1$ and declination $\delta=-6^{\circ} \pm 2^{\circ}$ (galactic latitude $b=50^{\circ} \pm 2^{\circ}$ and longitude $l=266^{\circ} \pm 3^{\circ}$ ). The weighted average magnitude found in Section 6.1 .1 corresponds to a velocity of $367 \pm 13 \mathrm{rm} / \mathrm{sec}$ in essentially the sane directicn. This velocity can be decomposed into several components at different levels of aggregation. The sun moves relative to the "local standard of rest" defined by nearby stars, the closest of which are on the order of parsecs away. This ensemble participates in galactic rotation about the galactic center some 10 kiloparsecs distant. The Milky Way galaxy is a member of the "Local Group", a collection of ten to twenty primarily dwarf galaxies within about a megaparsec. The entire Local Group belongs to yet a larger structure, the equally prosaicallynamed Local Supercluster, which is dominated by the Virgo cluster of galaxies some 15 Mpc away. Oply the vector sum of all the relative velocities is measured by the CBR anisotropy.

Referring the sun's velocity $v_{\odot}$ to the centroid of the Local Group by subtracting their relative motion $v_{\Theta}-v_{L G}$ of $308 \mathrm{~km} / \mathrm{sec}$ towards $(\alpha, \delta)=\left(22 .{ }^{h} 9,51^{\circ}\right) ;(l, b)=\left(105^{\circ},-7^{\circ}\right)($ Yahil et al., 1977). the $90 \mathrm{GHz}$ anisotropy shows the Local Group to have a velocity of $640 \mathrm{~km} / \mathrm{sec}$ towards $(\alpha, b)=\left(11 .^{h} 1,-26^{\circ}\right)$. This direction is $43^{\circ}$ away from the Virgo cluster centered at $(\alpha, \delta)=\left(12 .{ }^{h}, 13^{\circ}\right)($ Sandage and Tammann, 1976), yielding a velocity component towards Virgo of $470 \pm 30 \mathrm{~km} / \mathrm{sec}$. 
Studies of Local Group velocity relative to objects closer to us than the last scatterers of the CBR are required in order to decouple currents within the Loca Supercluster from large-scale bulk flow of the entire supercluster. Sevcral such studies bave been made, but they are not all in agreement. They are reviewed in a recent paper by Davis and Peebles (1983b) which discusses anisotropy in the Hubble flow.

Anisotropy in the hard I-ray background has been observed which is not inconsistent with the CBR. measurements. However, the sources of the $x$-ray background are not well understood. If the $\mathrm{x}$-rays are produced at the present epoch, then an increased concentration of sources in the local supercluster proportional to the observed enhancement of optical luminosity could account for most of the x-ray asymmetry (Boldt, 1981).

Three studies of solar velocity derived from anisotropy in galactic red shifts are compared with the velocity relative to the CBR in Table 6.5. Using luminosity as a distance indicator, Rubin et al (1976) examine spiral galaxies outside the Local Supercluster and assign thr sun a peculiar velocity of $600 \mathrm{~km} / \mathrm{sec}$ towards $(\alpha, \delta)=\left(2 .{ }^{h} 1,53^{\circ}\right)$. The Sc galaxies studied have recession velocities between 3500 and $6500 \mathrm{~km} / \mathrm{sec}$, corresponding to distances of $35 h^{-1}$ to $65 h^{-1}$ Mpc with the Hubble constant $H_{0}$ expressed as $100 \mathrm{hm} \mathrm{sec}-1 \mathrm{Mpc}^{-1}$. Removing the solar velocity, Rubin et al. find their data is consistent with isotropic Hubble expansion. 
Toble 6.5. Solar peculiar velocity relative to the CBR and to three samples of galaxies. Velocity direction is given both in celestial and galactic coordinates. No correction for solar motion within the galary and Local Group has been applied.

\begin{tabular}{|c|c|c|c|c|}
\hline & This Work & $\begin{array}{c}\text { Rubin et al., } \\
1976\end{array}$ & $\begin{array}{c}\text { De Vaucouleurs et al., } \\
1981\end{array}$ & $\begin{array}{c}\text { Hart and Davies, } \\
1982\end{array}$ \\
\hline $\begin{array}{c}\text { Magnitude: } \\
(\mathrm{km} / \mathrm{sec})\end{array}$ & $38 \mathrm{i} \pm 25$ & $600 \pm 125$ & $306 \pm 40$ & $310 \pm 55$ \\
\hline $\begin{array}{c}\alpha: \\
(\text { hours })\end{array}$ & $11.3 \pm 0.1$ & $2.1 \pm 1.3$ & $17 \pm 7$ & $12.3 \pm 0.7$ \\
\hline $\begin{array}{c}\delta: \\
(\mathrm{deg})\end{array}$ & $-6 \pm 2$ & $53 \pm 11$ & $84 \pm 7$ & $30 \pm 12$ \\
\hline $\begin{array}{c}\text { l: } \\
(\mathrm{deg})\end{array}$ & $266 \pm 3$ & $135 \pm 12$ & $117 \pm 12$ & $192 \pm 60$ \\
\hline $\begin{array}{c}b: \\
(\mathrm{deg})\end{array}$ & $50 \pm 2$ & $-8 \pm 12$ & $30 \pm 7$ & $83 \pm 12$ \\
\hline
\end{tabular}

De Vaucouleurs et al. (1981) study the motion of 300 spiral galaxies receding at between 200 and $2900 \mathrm{~km} / \mathrm{sec}$. Assuming isotropic expansion and fitting observed galactic red shifts to the dipole anisotropy which would be generated by a solar peculiar velocity, they find a relative motion between the sun and the galaxy sample of $306 \pm 40 \mathrm{~km} / \mathrm{sec}$ towards $(\alpha, \delta)=\left(17^{h}, 84^{\circ}\right)$. Galactic distances are determined by the Tully-Fisher method of comparing the observed optical luminosity to the absolute luminosity determined from the hydrogen 21-cm linewidth. Solutions excluding galaxies near the supergalactic center are consistent with the results of the full sample.

Hart and Davies (1982) analyze a sample of $78 \mathrm{Sbc}$ galaxies receding at between 1000 and $5000 \mathrm{~km} / \mathrm{sec}$. The redshift of the hydrogen $21-\mathrm{cm}$ line provides velocity data, and the line's 
legrated flux, parameterized by its width, serves as a standard candle. Their result for solar peculiar velocity is $310 \pm 55 \mathrm{~km} / \mathrm{sec}$ towards $(\alpha, \delta)=\left(12 .^{h} 3,30^{\circ}\right)$.

The augular resolutions of the peculiar velocity studies relative to galaxies are on the order of t.en degrees. None of the velocity directions in Table 6.5 agree to that level. The Hart and Davies velocity is closest to the velocity implied by the CBR anisotropy at $90 \mathrm{GH} \varepsilon$, with a difference between the directions of two velocities of 39 degrees. Discrepancies in magnitude among the studies make the disagreement even worse. After being referred to the centroid of the Local Group, the Hart and Davies finding shows the Local Group to have a velocity $463 \mathrm{~km} / \mathrm{sec}$ directed $26^{\circ}$ from Virgo. The direction of this velocity is $17^{\circ}$ from the Local Gruup's velocity with respect to the CBR, with the difference vector between Hart and Davies velocity and the CBR velocity having magnitude $242 \mathrm{kno} / \mathrm{sec}$. Inconsistency among Local Group velocities relative to different sets of galaxies wonld imply either relative motion between the galaxy sets or else underestimated systematic error. Disagreement between galactic studies and CBR anisotropy similarly implies either large-scale motion of the supercluster as a whole, or systematic error.

The Rubin result, in particular, is highly controversial. Fall and Jones (1976) argue that. inhomogeneity in the distribution of galazies (inferred from the known inhomogenous distribution of clusters), together with use of a narrow magnitude window, can cause selection of galaxies in an isotropically expanding universe which will display an anisotropic velocity distribution. Hart and Davies (1982) repeat their $21-\mathrm{cm}$ line analyses on those Sc galaxies in the Rubin et al. sample which have recession velocities between 1000 and $5000 \mathrm{~km} / \mathrm{sec}$, augmented by some additional southern hemisphere galaxies. They find a result consistent with their own (Hart and Davies) Sbe galazy sample and inconsistent with the Rubin et al. flndings. In their review, Davis and Peebles (1983b) lend greater credence to studies such as Hart and Davies (1982) which employ an additional distance-independent variable such as 21-cm linewidth.

The similarity of the Hart and Davies velocity to the CBR-derived motion suggests 
that the dipole anisotropy could be caused be motion within the supercluster, although with a several standard-deviation discrepancy remaining this conclusion is certainly not required. In supercluster internal-flow models, the component of velocity towards Virgo results frow: the gravitational attraction of the enhanced matter distribution in that direction, while a transverse component could arise from a lack of spherical symmetry in the supercluster collapse (Davis and Peebles, 1983b). The Local Grouf velocity with ?espect to the CBR need not point directly towards Virgo if this is the case. Alternatively, discrepancy between the CBR anisotropy and the Milky Way motion within the supercluster implies either an intrinsic component to the CBR anisotropy or the existence of bulk flow on larger scales than have yet been exarained.

\subsection{Quadrupole Anisotropy}

This experiment detects no significant quadrupolar anisotropy in the CBR. It does not reproduce the "quadrupole-like" anisoiropy of Fabbri et al., (1980) nor the significant quadrupole terms reported in Boughn et al. (1981), which were attributed in a later publication by that group to ground radiation and excess radiometer noise synchronous witi gondola rotation (Fixsen et al., 1983). Table 6.6 presents the quadrupole coefflients reported by the experiments whose dipole terms are given in Table 6.2. In the sbseare of true quadrunolar anisotrogy: an experiment with statistical errors and correlations equivalent to those of this work would measure quadrupole power equal to or greater than that detected here $45.5 \%$ of the time.

A limit on quadrupolar anisotropy can be quantifled by defining the average quadrupole temperature

$$
\begin{aligned}
T_{Q, \mathrm{rms}} & =\left(\frac{1}{4 \pi} \int_{4 \pi} T_{Q}^{2}(\Omega) d \Omega\right)^{\frac{1}{2}}, \text { where } \\
T_{Q}(\Omega) & =\sum_{i=1}^{5} Q_{i} q_{i}(\Omega)
\end{aligned}
$$


Table 6.6. Comparison of quadrupole coefilcients in mK thermodvnamir temperature.

\begin{tabular}{|c|c|c|c|c|c|}
\hline Reference & $Q_{1}$ & $Q_{2}$ & $Q_{3}$ & $Q_{4}$ & $Q_{5}$ \\
\hline This work & - & $0.15 \pm 0.14$ & $0.16 \pm 0.14$ & $-0.06 \pm 0.10$ & $0.09 \pm 0.09$ \\
\hline $\begin{array}{l}\text { Fixsen et al., } \\
1983\end{array}$ & $0.15 \pm 0.08$ & $0.15 \pm 0.11$ & $0.14 \pm 0.07$ & $0.06 \pm 0.11$ & $-0.01 \pm 0.07$ \\
\hline $\begin{array}{l}\text { Smoot and Lubin, } \\
1979\end{array}$ & $0.38 \pm 0.26$ & $-0.34 \pm 0.29$ & $0.02 \pm 0.24$ & $-0.11 \pm 0.16$ & $0.06 \pm 0.20$ \\
\hline $\begin{array}{l}\text { Boughn et al. } \\
\quad 1981\end{array}$ & - & $0.28 \pm 0.22$ & $0.13 \pm 0.21$ & $-0.31 \pm 0.15$ & $-0.54 \pm 0.14$ \\
\hline
\end{tabular}

and the quadrupole basis functions $q_{i}$ are defined in Table 5.1. Evaluating the angular integrals,

$$
T_{Q, \mathrm{rms}}^{2}=\frac{1}{5} Q_{1}^{2}+\frac{4}{15} \sum_{i=2}^{5} Q_{i}^{2} .
$$

Since this experiment is insensitive to $Q_{1}$, only four of the five quadrupole degrees of freedom can be examined. Assuming that the universe is not aligned with the earth's north pole, the total quadrupole power $\left(\sim T_{Q, r m s}^{2}\right)$ can be bounded by augmenting the limit on power in the measured $Q_{2}$ to $Q_{5}$ terms by an additional $25 \%$.

Neglecting $Q_{1}$, the value $T_{Q_{1} \text { rms }}$ in antenna temperature for the $90 \mathrm{GHz}$ data is $0.10 \mathrm{mK}$. Monte Carlo calculations show that ninety percent of the time, a radiometer having the statistical errors and correlations of this experiment and observing a true quadrupole anisotropy given by the $Q_{2}$ to $Q_{5}$ values in Table 5.9 will have $T_{Q, r m s} \leq 0.20 \mathrm{mK}$. The calculation involves diagonalizing the error matrix to express the quadrupole coefficients in uncorrelated basis functions, jittering each transformed coefflent with noise values taken from a distribution whose variance is the coefficient's mean-square error, histogramming the resultant values of $T_{Q . r m k}$, and finding that value marking the $90^{\text {th }}$ percentile of $T_{Q, \text { rms }}$ distribution. Multiplying this value by the square root of 1.25 to account for the missing $Q_{1}$ power gives a $90 \%$ confidence-level upper limit. of $0.22 \mathrm{mK}$ antenna temperature. The equivalent thermodynamic rms quadrupole upper limit for 
$T_{\mathrm{CBR}}=2.7 \mathrm{~K}$ is $0.27 \mathrm{mK}$, or a part in $10^{-4}$ of the CBR.

The rms dipole anisotropy is given by an analogous formula,

$$
\begin{aligned}
T_{D, \mathrm{rms}} & =\left(\frac{1}{4 \pi} \int_{4 \pi} T_{D}^{2}(\Omega) d \Omega\right)^{\prime} \\
& =\left(\frac{\left(T_{x}^{2}+T_{y}^{2}+T_{z}^{2}\right)}{3}\right)^{\prime} .
\end{aligned}
$$

Substituting the values in Table 5.9, the rms dipole anisotropy $T_{D, r m s}=2.01 \mathrm{mK}$ (thermodyoamic), yielding $\left(T_{Q, r m s} / T_{D, r m s}\right) \leq 0.13$ at $90 \%$ confldence.

Fixsen et al. (1983) present values for all five quadrupole components, so $F_{Q, r m s}$ can be computed directly from equation (6.4). The $90 \%$ confidence-level limit calculated from those components by the Monte Carlo technique described above is $0.23 \mathrm{mK}$ (thermodynamic). An alternative method of comparing the quadrupole limit of Fixsen et al. to this work is to resolve the Fixsen et al. system without a $Q_{1}$ term. Following the identical procedure on the re-determined error and solution parameters as was performed on the $90 \mathrm{GHz}$ results gives a $90 \%$ confidence-level quadrupole temperature limit of $0.22 \mathrm{mK}$ for the Fixsen et al. data. The $90 \%$ limit actually quoted by Fixsen et al. is $0.19 \mathrm{mK}$, but the method of analysis is not explicitly described.

\subsection{Galactic Emission}

Fits to the $90 \mathrm{GHz}$ data are unaffected by inclusion of the galactic plane (Table 5.6), showing that the data are not severely contaminated by dust emission. At $24.5 \mathrm{GHz}$, Fhere galactic emission is much stronger, failure to model or exclude the galayy changes the fitted $T_{y}$ component of the dipole by almost $0.5 \mathrm{mK}$ (Fixsen, 1083). Fits of the $90 \mathrm{GHz}$ data to galactic models are consistent with dust emission, averaged over the region within five degrees of the galactic plane and convolved with the radiometer beam, of alout $0.5 \mathrm{mK}$. This value represents 
a global fit to the entire observed sky; dust emission in any speciflc region can be estimated only to the extent that the overall galactic model correctly refects the dust distribution in that region.

\subsection{Summary}

The dipole anisotropy measured by this experiment at $90 \mathrm{GBz}$ has an antenna temperature magnitude of $2.82 \pm 0.19 \mathrm{mK}$ directed towards $11.3 \pm 0.1$ hours right ascension and $-5.7 \pm 1.8$ degrees declination. The direction is consistent with lower frequency observations, and the dipole magnitude measured by this experiment relative to that observed at $24.5 \mathrm{GHz}$ by Fixsen et al.is consistent with motion through a $2.7 \mathrm{~K}$ blackbody CDR. With that CBR spectrum, the thermodynamic anisotropy measured by this experiment bas magnitude $3.48 \pm 0.24 \mathrm{mK}$, corresponding to a solar velocity of $387 \pm 25 \mathrm{~km} / \mathrm{sec}$. Correcting for the sun's motion relative to the Local Group gives a Local Group peculiar velocity of $640 \mathrm{~km} / \mathrm{sec}$ directed $43^{\circ}$ from the Virgo cluster. Motion solely within the Local Supercluster may be responsible for the entire CBR anisotropy, but the discrepancies among studies of Local Group velocity with respect to ot Ler galasies make it impossible to drav this conclusion with high confldence.

This experiment finds no evidence for higher order anisotropy in the cosmic background radiation. The universe appears isotropic on the largest directly accessible scale, with a $90 \%$ confidence-level upper limit of $10^{-4}$ on the CBR quadrupole anisotropy. Since galactic emission at $90 \mathrm{GHz}$ is low, it is not a signiflcant source of systematic error in this experiment.

In the two decades since the discovery of the CBR, our understanding of the nature and history of our universe has increased tremendously. Angular anisotropy measurements, having improved in sensitivity by more than three orders of magnitude, characterize a dipole anisotropy which still has no detectable frequency dependence or higher order moments. 
Appendia A

\section{Relativistic Flux Transformation}

\section{A.1 Phase Space Density}

The intensity $I_{\nu}$ (ergs $\mathrm{cm}^{-2} \mathrm{sec}^{-1} \mathrm{~Hz}^{-1} \mathrm{str}^{-1}$ ) of a radiation fleld at frequency $\nu$ can be shown to be proportional to $\nu^{3}$ times the Lorentz-invariant photon phase space density $\eta$. Intensity transformation from one reference frame to anotier is therefore given in terms of the transformation of $\nu^{3}$.

Phase space density invariance follows from the invariance of the phase space volume element - if a group of particles occupies the same amount of phase space volume in all frames, all observers will measure the same value for the density. As shown below, although tbe different observers measure different physical space volumes $d x d y d z$ and momentum space volumes $d p_{z} d p_{y} d p_{z}$, the product $d^{3} z d^{3} z$ is invariant.

Consider first a group of massive particles occupying physical space element $d \tau d y d z$ in its rest frame. An observer moving along the $x$ direction with velocity $\beta=v / c$ measures transverse dimensions $d y^{\prime}$ and $d z^{\prime}$ equal to the rest-frame dimensions $d y$ and $d z$. Her measurement of the longitudinal dimension $d x^{\prime}$ is made at a single instant. in her own frame, so $d t^{\prime}=0$ and $d x=\gamma\left(d x^{\prime}-\beta c d t^{\prime}\right)=\gamma d x^{\prime}$. Her Lorentz-contracted length measurement, multiplied by 
the unchanged area, gives a physical-space volume compressed by a factor of $\gamma$ relative to the particle rest frame: $d p_{x} d p_{y} d p_{x}=d^{3} \vec{z}^{\prime}=d^{3} \vec{x} / \gamma$.

Since kinetic energy is quadratic in momentum, the spread in particle energy $c^{2} d p_{0}$ vanishes (i.e. is of order $d p_{x}^{2}$ ) in the particle rest frame. The moving observer measures $c d p_{x}^{\prime}=$ $\gamma\left(c d p_{x}-\beta c^{2} d p_{0}\right)=\gamma c d p_{x}$. Again, the transverse measurements $d p_{y}^{\prime}=d p_{y}$ and $d p_{z}^{\prime}=d p_{z}$ are unaffected, so $d^{3} z^{\prime}=\gamma d^{3} Z$. The total phase space volume is seen to be

$$
\begin{aligned}
d^{3} \vec{x}^{\prime} d^{3} \vec{z}^{\prime} & =\left(\frac{d^{3} \vec{z}}{\gamma}\right)\left(\gamma d^{3} \vec{z}\right) \\
& =d^{3} \vec{z} d^{3} \underline{x}
\end{aligned}
$$

for all observers. In the limit as rest mass approaches zero, the identity remains valid, establishing phase space volume and density invariance for photons as well.

\section{A.2 Intensity}

Given a photon phase space density, the intensity measured by an observer will depend on the energy of each detected photon and the rauge of solid angle subtended by the photons. The energy per photon is proportional to $v$, and the amount of solid angle per unit momentum space goes as $\nu^{-2}\left(d^{3} \vec{z}=p^{2} d p d \Omega\right.$, with $p=|\vec{x}| \sim \nu$ for photons, so $\left.d \Omega / d^{3} \vec{x} \sim \nu^{-2}\right)$. Therefore, the intensity is proportional to $\nu^{3}$ times the phase space density $\eta$. (Misner et. al, 1973, p. 589) The ratio $I_{\nu} / \nu^{3}$, proportional to $\eta$, is Lorentz invariant and can be used to transform radiation intensity:

$$
\begin{aligned}
& \frac{I_{\nu}^{\prime}}{I_{\nu}}=\left(\frac{\nu^{\prime}}{\nu}\right)^{3} \\
& I_{\nu}^{\prime}=\left(\frac{\nu^{\prime}}{\nu}\right)^{3} I_{\nu} .
\end{aligned}
$$

If a radiation fleld is isotropic in the unprimed frame, this relation gives its angular dependence for an observer in the moving primed frame. Let $\theta$ be the angle in the unprimed 
frame between the direction from which the photon arrives and the moving observer's velocity. A photon baving angular frequency $\omega$ and wavevector $\vec{k}$ in the isotropic frame ( $\mathbf{w i t h} \omega=|\vec{k}| c$ ) will have

$$
\begin{aligned}
\omega^{\prime} & =\gamma\left(\omega-\beta c k_{x}\right) \\
& =\gamma \omega(1+\beta \cos \theta) \\
& =\omega(1+\beta \cos \theta) \operatorname{for} \beta<<1,
\end{aligned}
$$

so for small velocity, $\left(\nu^{\prime} / \nu\right)=\left(\omega^{\prime} / \omega\right)=(1+\beta \cos \theta)$.

For a spectrum having $\operatorname{logarithmic}$ frequency derivative $(\theta \log l / \delta \log \nu)$ equal to $\alpha$ near fr:quency $\nu_{0}$ such that $I \sim J_{0}\left(\nu / \nu_{0}\right)^{\alpha}$, we obtain

$$
\begin{aligned}
I_{\nu}\left(\theta^{\prime}\right) & =\left(\frac{\nu^{\prime}}{\nu}\right)^{3} I_{0}\left(\frac{\nu}{\nu_{0}^{\prime}}\right)^{\alpha} \\
& =I_{0}\left(\frac{\nu^{\prime}}{\nu}\right)^{3}\left(\frac{\nu}{\nu^{\prime}}\right)^{\alpha}\left(\frac{\nu^{\prime}}{\nu_{0}}\right)^{\alpha} \\
& =\left[I_{0}\left(\frac{\nu^{\prime}}{\nu_{0}}\right)^{\alpha}\right]\left(\frac{\nu^{\prime}}{\nu}\right)^{3-\alpha} \\
& =I_{0}^{\prime}\left(1+\left(3-\sigma_{0}\right) \beta \cos \theta\right)
\end{aligned}
$$

for observations at a fixed frequency $\nu^{\prime}$ in the primed frame. 
Appendix B

\section{Radiometers}

A micyowave radiometer mensures electromagnetic energy. The simplest type of radjometer measures the total power collected by an antenna. Added to the amplifled input power is noise generated withi' the radiometer itself, so the output of a total power radiometer is a signal proportional to the sum of the incident and the interually-generated porer. The system noise temperature characterizes noise generated by the radiometer. It is the antenna temperature of a source which, when observed by a noise-free radiometer, produces the same output as the actual radiometer would yield looking at source at zero $\mathbf{K}$.

The radiometer output will fluctuate due to statistical variation : . . ne noise power and to fluctuations in the radiometer gain. These output fluctuations determine the resolit:ion with which the input antenna temperature can sa measured. For a total power radiometer with system noise temperature $T_{\text {syz }}$, the RMS output fluctuations when observing incident antenna temperature $T_{\mathrm{a}}$ are

$$
\Delta T=\left(T_{\mathrm{sys}}+T_{\mathbf{2}}\right)\left\{\left(\frac{1}{B \tau}\right)+\left(\frac{\Delta G}{G}\right)^{2}\right\}^{\frac{1}{2}},
$$

where $B$ is the bandwidth, $\tau$ is the net integration time, and $(\Delta G / G)$ is the RMS fractional change in recciver gain over the integration time. $\Delta T$, also known as the figure of merit, is the input signal which can be detected with a signal-to-noise ratio of unity. 
To minimize the effect of gain fluctuations, a differential radiometer (Dicke, 1045) switches between the source anci a reference and yjelds an outpu proportional to their differeuce in emitted power. If the source has exactly the same intensity as the reference, the radion etes is balanced and has no net output. Gain variations on time scal:s longer than tie switching feriod in a balanced differential radiometer are cancelled out. If there is a- IIset temperature between source and reference, gain variations will cause somc fluctuation in the output. Hu-gever, these Euctuations will be suppressed by a factor of $T_{\text {offet }} /\left(T_{s w s}+T_{\mathrm{a}}\right)$ with respect to those of a total power radions. $r$. If the reference temper ature cannot be adjusted to eliminate the offset, the effect of gain variacion on the offset can be minimized $b_{y}$ iaterchanging source and reference, as is done when the $90 \mathrm{GP}$. radiou eter rotates $180^{\circ}$. This interchange introduces a further level of diferencing, which nulls out gain varititions on time scales longe; than the interchange period.

The sencitivity of a differential radjometer having offset temperature $T_{\text {offset }}$ is given by the expression

$$
\begin{aligned}
\Delta T=\left[\left\{\left(T_{\text {sys }}+T_{\mathrm{a}}\right)\left(\frac{K}{\sqrt{B \tau}}\right)\right\}^{2}\right. & +\left\{\left(T_{\text {sys }}+T_{\mathrm{a}}\right)\left(\frac{\Delta G}{G}\right)_{\substack{\text { switcb } \\
\text { period }}}\right\}^{2} \\
& \left.+\left\{\left(T_{\text {offet }}\right)\left(\frac{\Delta G}{G}\right)_{\substack{\text { inter- } \\
\text { chagge }}}\right\}^{2}+\left\{\left(\Delta T_{\text {offsel }}\right)\right\}^{2}\right]^{\frac{1}{2}}
\end{aligned}
$$

where the constant $K$ der,ends on how the synchronous switching and detection are done, and $\Delta T_{\text {offet }}$ in, udes drift in the reference temperature. The $(\Delta G / G)$ terms are RMS fractional gain variations over the indicated time periods. If source and reference are not interchanged, the "interchange period" becomes the total observation time.

For an ideal differential radiometer, the factor $K$ in equation (B.2) is 2. The factor comes about because a differential radiometer observes the source only half the time, spending the remainder on the reference. The statistical fluctuations in source and reference measurements are greater by $\sqrt{2}$ than those of a total power radiometer spending the entire time on one or the 
other, and fluctuations in differencing the source and reference intensities are another factor of $\sqrt{2}$ greater than those in either measurement alone. In practice, $K$ also depends on details of the synchronous flter design such as the number of harmonics of tin. switching frequency which are transmitted by stages downstream of the switch. Since the $90 \mathrm{GHz}$ lockin back end passes several switching-frequency harmonics, its value for $K$ should be very close to 2 .

Two references which discuss descriptions and properties of different types of microwave radiometers are Etrans and McLeish, 1977, and Kraus, 1966. 
Appendix C

Calibration

\section{C.1 Primary Calibration}

Primary calibration of the radiometer in the laboratory and on the flight line is done by positioning a blackbody targe: removed from a container of liquid nitrogen in one beam and an ambient-temperature blackbody in the other. The targets are held in position until the lockin output stabilizes, and the output is recorded before the liquid nitrogen target starts to warm up (typically within 10 to 15 seconds). Averaging the difference in outputs between measurements with the targets interchanged remores any ofiset. The ambient temperature is measured either by a thermometer or by temperature sensors on the radiometer. Ambient temperature uncertainty of two degrees yields a calibration error of one percent and is not a significant source of error. The liquid nitrogen 1 et is assumed to be at the temperature of boiling liquid nitrogen at sea level. The slope of the boiling curve is $0.085 \mathrm{~K}$ per percent change in pressure, so atmospheric pressure variations of up to $5 \%$ introduce less than $0.5 \mathrm{~K}$ uncertainty in the liquid nitrogen temperature. Measurements at $10 \mathrm{GHz}$ and $33 \mathrm{GHz}$ show that an Eccosorb ${ }^{1}$ target dipped in and then removed from liquid nitrogen has an antenna temperature within $\pm 0.3 \mathrm{~K}$ of a target

\footnotetext{
${ }^{1}$ Emerson and Cummings microwave absorber model $\mathrm{AN}-73$
} 
immersed in liquid nitrogen (S. Friedman and G. De Amici, private communication). For the purposes of this experiment, the $77.4 \mathrm{~K}$ boiling point $T_{\mathrm{LN}}$ of liquid nitrogen is taken to be $77 \mathrm{~K}$.

The primary calibration value, averaged over widely separated measurements in a threeweek period before the November light, was 67.2 Kelvins per lockin output volt with an RMS seatter of $\mathbf{2 . 6 \%}$. No changes affecting system gain were made following that flight, and a corresponding average of calibrations done over two weeks before the April flight was $67.6 \mathrm{~K} /$ volt with a scatter of $2.1 \%$. The weighted average of November and April values, $67.5 \mathrm{~K} / \mathrm{volt}$, was used in analyzing data from both flights. Calibrations done on the flight line immediately before each flight $(66.9 \pm 0.9 \mathrm{~K} /$ volt in November and $67.6 \pm 0.5 \mathrm{~K} /$ volt in April; both included in the averages above) were consistent with the laboratory measurements.

\section{C.2 Corrections}

Two corrections were made to the calibration constant to yield the value used on flight data. The first of these accounts for the change in lockin gain by a factor of $101.1 \pm 0.2$ between the scales used for ground calibration (range $\pm 338 \mathrm{~K}$ ) and for flight (range $\pm 3.34 \mathrm{~K}$ ). The second corrects for slight saturation of the detector diode. If the diode characteristic (voltage out vs. power in) becomes non-linear, jts slope is a function of the incident power level. The above calibration procedure measures the slope of the chord connecting the operating points for ambient temperature and liquid nitrogen temperature loads. For a non-linear characteristic, this slope is not necessarily the same as the slope of the characteristic at the operating point maintained by the system when viewing the $3 \mathrm{~K} \mathrm{CBR}$.

To determine the slope at the actual operating point, power in the radiometer IF band was att.enuated by varying amounts and detected. The resultant diode output voltages were fitted to a cubic polynomial in incident power level. This polynomial was used to find analytically the ratio of the slope of the chord connecting the ambient and $L N$ calibration points to the slope 
of the characteristic at the high-adtitude operating point. The calibration constant measured with ambient and $L N$ loads, in Kelvins per volt, is inversely proportional to the slope of the $\left(T_{\text {amb }}-T_{\mathrm{LN}}\right)$ chord. Multiplying the measured calibration constant by the ratio fonnd above, and correcting for the change in gain, yields the calibration constant ased at the flight operating point.

The diode characteristic calculated for the $90 \mathrm{GHz}$ radiometer showed a $4.5 \%$ nonlinearity for an ambient temperature load, a $3.4 \%$ non-linearity for liquid nitrogen, and $1.3 \%$ for the operating point at altitude. The ratio of the slope of the $\left(T_{\text {amb }}-T_{\text {LN }}\right)$ chord to the slope at the operating point is 0.96 with an estimated error of \pm 0.02 . Using this factor and correcting for the difference in gain, the conversion at altitude from lockin output volts to milliKelvins antenna temperature is $641 \mathrm{mK} /$ volt.

\section{C.3 Stability of and Error in Calibration}

\section{C.3.1 Pop-up Calibrator - Description}

The in-fight pop-up calibrator is a piece of iron-loaded epoxy microwave absorber $0.35 \mathrm{~cm}^{2}$ in area, mounted with a temperature sensor on the end of a brass rod $0.31 \mathrm{~cm}$ wide. When raised into the beam, the target subtends a solid angle of $1.9 \times 10^{-4}$ steradians at a distance trom the horn of $43 \pm 3 \mathrm{~cm}$, depending upon where on the horn the measurement is taken. At that distance, not quite into the far-fleld of the antenna (which is distances greater than $\pi a^{2} / \lambda$ from a circular horn of radius $a$, or $60 \mathrm{cr}$ : for this experiment), the horn's gain pattern is not known accurately enough to use the target as an absolute calibration. As a first approximation, the ratio of the target solid angle to the antenna's integrated gain-weighted solid angle of $0.021 \pm 0.003$ steradians predicts that the target. will generate an antenna temperature equal to $0.009 \pm 0.0013$ of its physical temperature. 
Seen from the horn and conrolved over the same antenna pattern, the support rod subtends $0.0214 \pm 0.015$ of the beam, or 2.4 times that of the target. The emissivity of brass is on the order of $10^{-3}$, so emission from the arm is negligible. As long as the variations in radiation scattered of the arm are small compared to the target emission, a constant calibrator signal will indicate a stable system calibration. If the scattered contribution is variable, changes in calibrator signal amplitude provide an upper bound to system variability.

\section{C.3.2 Pop-up Calibrator Flight Observations}

In-fight calibrator observations were made every 27 minutes during the Norember, 1981 hight. Each was analyzed by taking the average of six 2.1-second integration periods during which the calibrator was centered in the direct beam, and subtracting the mean of equivalent sections before and after the calibration. The statistical uncertainty of each observation was $0.006 \mathrm{~K}$ or $0.2 \%$. During the data-taking portion of the flight, the calibrator temperature varied by $1.4 \%$ between 219 and $222 \mathrm{~K}$.

The mean magnitude of the calibrator signals for the November flight was $2.26 \mathrm{~K}$, wit.h an RMS scatter of $0.014 \mathrm{~K}$ or $0.6 \%$. The predicted calibrator emission is $1.96 \pm 0.33 \mathrm{~K}$, with the error representing uncertainty in the target's true gain-weighted solid angle. Besides emission, power scattered of the arm may have contributed to the signal as well. The total signal generated by the calibrator therefore consisted of emission from the target plus scattering off the arm, minus the sky emission which was blocked iy the target and arm. At altitude, the arm sees primarily sky or reflected sky with an angle-independent intensity. Except for power scattered from the earth, radiation scattered by the arm has the same antenna temperature as radiation blocked by the arm. Groundshields prevented most earth emission from reaching the arm and target, although the arm and target could see the borizon in the November, 1981 conflguration. During the 13 seconds that the target was in the beam each calibration, the gondola rotated through 85 
degrees. Therefore, horizon power scattered by the calibrator was averaged over a large area.

\section{C.3.3 Pop-up Calibrator Ground Observations}

Measurements done on the ground could not unambiguously resolve the level to which scattering influenced the in-flight calibrations. The sky was rarely stable enough to permit observations of the pop-up target on the ground using the flight sensitivity. In addition, the $\sec \theta$ variation of atmospheric temperature with zenith angle, along with the presence of warm objects of various sizes on the horizon, cause scattered power in ground observations to have in general a much different temperature than the atmospheric emission which is blocked by the arm. Therefore, scattering should have a greater effect on the calibrator signal in ground observations than it would at altitude.

Crude observations made before the first flight in July showed that the arm without target generated a signal about one-third of that generated by the arm plus target. This result indicates that on the ground, the difference in power scattered minus power obstructed by the arm alone is half the correspouding difference in power emitted minus power obstructed by the target.

Observations of the pop-up calibrator on the ground were made using the fight sensitivity on three widely spaced instances in Texas and in Berkeley. The calibrator physical temperature, sky temperature, and measured signal are shown in Table C.1 along with the predicted signal assuming the target solid angle given earlier. The solid angle is highly uncertain but is the same for all observations, so the differences in measured signal give an indication of the variability of the scattered contribution. Calibration constants measured at the time of each observation, along with saturation corrections appropriate to the sky temperature, have been used.

The large variation in signal (from 1.22 to 1.74 times the predicted ralue) probably 
Table C.1. Pop-up calibrator ground observations.

$\begin{array}{rcccc}\text { Date } & \begin{array}{c}\text { Calibrator } \\ \text { Temperature }\end{array} & \begin{array}{c}\text { Sky } \\ \text { Temperature }\end{array} & \begin{array}{c}\text { Predicted } \\ \text { Signal }\end{array} & \begin{array}{c}\text { Measured } \\ \text { Signal }\end{array} \\ 11 / 82 & 309 \pm 1 & 48 \pm 1 & 2.35 \pm 0.40 & 3.14 \pm 0.07 \\ 11 / 82 & 307 \pm 1 & 71 \pm 2 & 2.12 \pm 0.35 & 2.59 \pm 0.04 \\ 4 / 83 & 283 \pm 1 & 46 \pm 2 & 2.12 \pm 0.35 & 3.68 \pm 0.05\end{array}$

represents the differences in effective horizon temperature. The last measurement above, for example, was made in a parking lot surrounded by trees and buildings.

\section{C.3.4 Error in Calibration Constant}

In any given measurement of the system calibration, the temperature difference $T_{\mathrm{amb}}$ $T_{\mathrm{LN}}$ and the radiometer output voltage can both be measured to better than one percent. The dominant. source of uncertainty is variation arising from system drifts and differences in the beam filling factor for the liquid-nitrogen temperature. Scatter among widely-spaced calibration measurements gives an estimate of these effects and is under $3 \%$ for this system. Any systematic change in system behavior between ground and fight, or from flight to flight, will also directly contribute to caljbration error. To account for possible systematic errors, the absolute calit.ration uncertainty bas been taken to be $\pm 6 \%$ with a relative fight-to-flight uncertainty of $3 \%$. The pop-up in-flight calibrator was replaced with a full-beam calibrator following he April, 1982 fight; unfortunately data with observations of this calibrator at altitude were lost, along with the gondola, during the November, 1982 flight in Brazil. 
Appendir D

\section{Linear Least Squares Estimation}

\section{D.1 Best Fit Solution}

One of the most widely used techniques of modeling noisy data is least squares fitting, which determines the set of model parameters minimizing the squared residuals between the data and the model. Consider a collection of data measurements $y_{i}$, each having uncertainty $\sigma_{i}$. The measurements are to be modeled by a function of the $p$ to-be-determined parameters $a_{j}$ and the $l$ independent coordinates for each observation $\vec{x}_{i}=\left(x_{q}^{1}, \ldots, x_{i}^{l}\right)$.

Attributing all discrepancy between the best-flt model and the data to noise, we have

$$
y_{i}=W\left(a_{j}, \vec{x}_{i}\right)+\epsilon_{i},
$$

with the $\epsilon_{i}$ representing randomly distributed noise values. To determine the best-fit parameters $a_{j}$, define

$$
\chi^{2}=\sum_{i}\left(\frac{\epsilon_{i}}{\sigma_{i}}\right)^{2}=\sum_{i} \frac{\left(y_{i}-W^{\prime}\left(a_{j}, \vec{x}_{i}\right)\right)^{2}}{\sigma_{i}^{2}} .
$$

Minimize $\chi^{2}$ with respect to the $a_{j}$ :

$$
\frac{\partial \chi^{2}}{\partial a_{j}}=\left.0 \Rightarrow \sum_{i} \frac{y_{i}}{\sigma_{i}^{2}} \frac{\partial W}{\partial a_{j}}\right|_{x_{i}}=\left.\sum_{i} \frac{W\left(a_{j}, \vec{x}_{i}\right)}{\sigma_{i}^{2}} \frac{\partial W}{\partial a_{j}}\right|_{\vec{x}_{i}}
$$


For models in which $W$ is linear in the parameters $a_{\jmath}$, the normal equations defined by (D.3) simplify considerably. If $W=\sum_{j} a_{j} f_{j}\left(\vec{x}_{i}\right)$, where the $f_{j}$ are a set of basis functions, then $\partial W / \partial a_{j}=f_{j}$, and the normal equations become

$$
\sum_{i} \frac{y_{i} f_{j}\left(\vec{x}_{i}\right)}{\sigma_{i}^{2}}=\sum_{i} \sum_{k} \frac{a_{k} f_{j}\left(\vec{x}_{i}\right) f_{k}\left(\vec{x}_{i}\right)}{\sigma_{i}^{2}}
$$

Deflne the data vector components $D_{j}$ to be $\sum_{i} y_{i} f_{j}\left(\vec{x}_{i}\right) / \sigma_{i}^{2}$ and the moment matrix $\mathbf{M}_{j k}=\sum_{i} f_{j}\left(\vec{x}_{i}\right) f_{k}\left(\vec{x}_{i}\right) / \sigma_{i}^{2}$. The normal equations become $D_{j}=\sum_{k} \mathbf{M}_{j k} \hat{a}_{k}$, and the best-fit solution parameters $a_{k}$ are found by inverting the system to give $\vec{a}_{k}=\sum_{j} \mathbf{M}_{j k}{ }^{1} D_{j}$.

\section{D.2 Uncertainty in Fitted Parameters}

The fitted $\hat{a}_{k}$ have an uncertainty due to noise in the observations $y_{i}$. The $\hat{a}_{k}$ are also in general correlated in that independent errors $\epsilon_{i}$ in the observations $\dot{y}_{i}$ will result in changes $\delta a_{k}$ to the solution vector components which are not independent. The uncertainties and correlations in the $\hat{a}_{k}$ can be analyzed by examining the expected value of the covariances $\left\langle\delta \hat{a}_{j} \delta \hat{a}_{k}\right\rangle$ over independent sets of measurement noise values $\epsilon_{i}$ :

$$
\begin{aligned}
\delta a_{j} & =\sum_{i} \frac{\partial a_{j}}{\partial \epsilon_{i}} \delta \epsilon_{i} ; \\
\left\langle\delta a_{j} \delta a_{k}>\right. & =<\sum_{i} \frac{\partial a_{j}}{\partial \epsilon_{i}} \delta \epsilon_{i} \sum_{i} \frac{\partial a_{k}}{\partial \epsilon_{l}} \delta \epsilon_{l}>.
\end{aligned}
$$

Now since $a_{i}=\sum_{m} \mathbf{M}_{j m}^{-1} D_{m}$, we have

$$
\begin{aligned}
\frac{\partial a_{j}}{\partial \epsilon_{i}} & =\sum_{m} \mathbf{M}_{j m}^{-1} \frac{\partial D_{m}}{\partial \epsilon_{i}} \\
& =\sum_{m} \mathbf{M}_{j m}^{-1} \frac{f_{m}\left(\vec{x}_{i}\right)}{\sigma_{i}^{2}}
\end{aligned}
$$

upon substituting equation (D.1) into the definition of the data vector. The covariances are 


$$
\left\langle\delta a_{j} \delta a_{k}\right\rangle=\sum_{i, l} \sum_{m, n} \mathbf{M}_{j m}^{-1} \frac{f_{m}\left(\vec{x}_{i}\right)}{\sigma_{i}^{2}} \mathbf{M}_{k n}^{-1} \frac{f_{n}\left(\vec{x}_{i}\right)}{\sigma_{l}^{2}}<\delta \epsilon_{i} \delta \epsilon_{l}>,
$$

and since the $\delta \epsilon_{i}$ are independent,

$$
\left\langle\delta \epsilon_{i} \delta \epsilon_{l}>= \begin{cases}\sigma_{i}^{2}, & \text { if } i=l \\ 0, & \text { otherwise }\end{cases}\right.
$$

Therefore

$$
\begin{aligned}
<\delta a_{j} \delta a_{k}> & =\sum_{m, n} \mathbf{M}_{j m}^{-1} \sum_{i} \frac{f_{m}\left(\vec{x}_{i}\right) f_{n}\left(\vec{x}_{i}\right)}{\sigma_{i}^{4}} \sigma_{i}^{2} \mathbf{M}_{k n}^{-1} \\
& =\sum_{m, n} \mathbf{M}_{j m}^{-1} \mathbf{M}_{m n} \mathbf{M}_{k n}^{-1} \\
& =\mathbf{M}_{j k}^{-1} .
\end{aligned}
$$

Hence the components of $\mathrm{M}^{-1}$, called the error matrix, give the solution parameter covariances, and the diagonal terms $\mathbf{M}_{j j}^{-1}$ are the mean squared errors of the components $a_{j}$ of the solution vector. The correlations between solution parameters are often expressed as a normalized correlation matrix $\mathbf{C}$ :

$$
\mathbf{C}_{j k}=\frac{\mathbf{M}_{j k}^{-1}}{\sqrt{\mathbf{M}_{j j}^{-1} \mathbf{M}_{k k}^{-1}}}
$$

\section{D.3 Comparison of Independent Solutions}

Consider parameter estimates $\hat{a}^{1}$ and $\hat{a}^{2}$ fltted to independent data sets. To determine Whether the fits are consistent with each other, examine the difference vector $\Delta \hat{a}=\hat{a}^{1}-\hat{a}^{2}$. If the two flts are solutions for the same observables, each component of this difference should have an expectation value of zero, with the expected covariance $\left\langle\left(\hat{a}^{1}-\hat{a}^{2}\right)_{j}\left(\hat{a}^{1}-\hat{a}^{2}\right)_{k}\right\rangle$ given by $\left\langle\left(\hat{a}_{j}^{1} \hat{a}_{k}^{1}\right)\right\rangle-\left\langle\left(\hat{a}_{j}^{1} \hat{a}_{k}^{2}\right)\right\rangle-\left\langle\left(\hat{a}_{j}^{2} \hat{a}_{k}^{1}\right)\right\rangle+\left\langle\left(\hat{a}_{j}^{2} a_{k}^{2}\right)\right\rangle$. The first and fourth terms, from (D.6), are $\mathbf{M}_{j k}^{1}{ }^{-1}+M_{j k}^{2}{ }^{-1}$. The cross terms are zero since the errors $\delta \epsilon_{i}^{1}$ and $\delta \epsilon_{l}^{2}$, which appear 
in the expression equivalent to (D.5) generalized to more than one data set, are independent: $<\delta \epsilon_{i}^{1} \delta \epsilon_{l}^{2}>=0$ even for $i=l$. Therefore,

$$
<\left(\hat{a}^{1}-\dot{a}^{2}\right)_{j}\left(\dot{a}^{1}-\dot{a}^{2}\right)_{k}>=\mathbf{M}_{j k}^{1}{ }^{-1}+\mathbf{M}_{j k}^{2}-1
$$

If the total error matrix $\mathbf{M}^{-1}$ is deflned such that $\mathbf{M}^{-1}=\mathbf{M}^{1-1}+\mathbf{M}^{-1}$, the covariance matrix of the $p$ elements of the difference vector $\Delta \dot{a}$ is $\mathbf{M}^{-1}$, and the scalar product

$$
S^{2}=\Delta \dot{a}^{T} \mathbf{M} \Delta \dot{a}
$$

will be distributed as $\chi^{2}$ for $p$ degrees of freedom. This distribution is easiest to see in a set of basis functions for which $\mathbf{M}$ is diagonal. $M$ is symmetric, so such a diagonalization can always be found. Since $\mathbf{M}^{-1}$ is the covariance matrix for the $\Delta \hat{a}_{j}$, which are uncorrelated in this basis, the elements $\mathbf{M}_{j k}^{-1}$ are equal to $\left\langle\Delta \ddot{a}_{j}^{2}>\right.$ for $j=k$ and 0 for $j \neq k$. Therefore, $\mathbf{M}_{j k}=\left\langle\Delta \hat{a}_{j}^{2}\right\rangle^{-1}$, and

$$
S^{2}=\sum_{j=1}^{p} \frac{\Delta \hat{a}_{j}^{2}}{\left\langle\Delta \bar{a}_{j}^{2}\right\rangle}
$$

which can be seen to have a $\chi_{p}^{2}$ distribution. The confidence level at which two independent solutions $\hat{a}^{1}$ and $\hat{a}^{2}$ agree is the confldence level of drawing $S^{2}$ from a $\chi^{2}$ distribution for $p$ degrees of freedom.

Two useful references discussing estimation and other statistical techniques are Bevington (1969) and Martin (1971). 
Appendix E

Generation of Sky Maps

\section{E.1 Normal Equations}

A map is a representation of the sky intensity distribution using a set of basis functions $f_{2}$ which have magnitude unity over a particular area or pixel in the sky, are zero elsewhere, and do not overlap. Relevant normal equations are derived by minimizing the following expression for $\chi^{2}$ (c.f. equation (D.2)):

$$
\chi^{2}=\sum_{i}\left(\frac{\Delta T_{i}-\left(T_{A i}-T_{B i}\right)}{\sigma_{i}}\right)^{2}
$$

where $T_{A i}$ and $T_{B i}$ are the temperatures of the pixel that radiometer beams $A$ and $B$ oiserve to give the $i^{\text {th }}$ difference $\Delta T_{i}$.

By adding all measurements linking a particular pair of pixels $j$ and $k$ to give an average temperature difference $\overline{\Delta T}_{j k} \pm \sigma_{j k}$, the sum over data points can be replaced by a double sum over pixels. Let $\{j\}$ be the set of pixels connected to pixel $j$ by at least one observation $\Delta T_{i}$. (Note that $j$ is not a member of $\{j i$, since no observation has pixel $j$ in both beams.) An equivalent formulation of $\chi^{2}$ becomes

$$
\chi^{2}=\sum_{j} \sum_{k \in\{j\}}\left(\frac{\overline{\Delta T}_{j k}-\left(T_{j}-T_{k}\right)}{\sigma_{j k}}\right)^{2} .
$$


The normal equations are given by setting $\left(\theta \chi^{2} / \partial T_{j}\right)=0$ for all $T_{j}$ :

$$
\begin{aligned}
\frac{\theta \chi^{2}}{\partial T_{j^{\prime}}} & =-2 \sum_{j} \sum_{k \in\{j\}}\left(\frac{\overline{\Delta T}{ }_{j k}-\left(T_{j}-T_{k}\right)}{\sigma_{j k}^{2}}\right)\left(\delta_{j}^{j^{\prime}}-\delta_{k}^{\prime}\right) \\
& =0, \quad \text { with } \delta_{j}^{j^{\prime}}= \begin{cases}1, & \text { if } j=j^{\prime} \\
0, & \text { otherwise. }\end{cases}
\end{aligned}
$$

We can eliminate the sum over $j$ and replace $\overline{\Delta T}_{k j}$ with $-\overline{\Lambda T}_{j k}$ to get

$$
\sum_{k \in\left\{j^{\prime}\right\}}\left(\frac{T_{j^{\prime}}-T_{k}-\overline{\Delta T}_{j^{\prime} k}}{\sigma_{j^{\prime} k}^{2}}\right)=0 .
$$

Expanding, and replacing $j^{\prime}$ with $j$,

$$
T_{j} \sum_{k \in\{j\}} \frac{1}{\sigma_{j k}^{2}}-\sum_{k \in\{j\}} \frac{T_{k}}{\sigma_{j k}^{2}}-\sum_{k \in\{j\}} \frac{\overline{\Delta T}_{j k}}{\sigma_{j k}^{2}}=\eta .
$$

By defining

$$
\begin{aligned}
a_{j j} & =\sum_{l \in\{j\}} \frac{1}{\sigma_{\jmath}^{2}} \\
a_{j k} & =-\frac{1}{\sigma_{j k}^{2}}, k \in\{j\} ;=r \text {, otherwise } \\
b_{j} & =\sum_{l \in\{j\}} \frac{\overline{\Delta T_{j l}}}{\sigma_{j l}^{2}}
\end{aligned}
$$

equation (E.3) reduces to

$$
\sum_{k} a_{j k} T_{k}=b_{j}
$$

The coefflcients $a_{j k}$ are non-zero only for pixel pairs connected by measurements $\Delta T_{i}$. If one radiometer beam is viewing a given pixel, there are only a very limited number of pixels which may be observed by the other beam. Therefore, the great majority of the $a_{j k}$ are zero, and the system (E.5) is quite sparse. Since $\overline{\Delta T}_{j k}=-\overline{\Delta T}_{k}$, implying $\sum, b,=0$, the equations (E.5 are not linearly independent. The data, all differential measurements. are insensitive to the addition of a constant to all pixels. An additional constraint equation is required to remove this degeneracy. 


\section{E.2 Gauss-Seidel Sparse Matrix Inveræion}

The Gauss-Seidel technique is an iterative method of inverting a system of linear equations. The system is expressed as a set of equations in whch each unknown pa ameter is given as a function of the others. If initial estimates are made for all parameters, an updated value for the first unknown can be calculated. This new value, along with the in:tial guesses for all the other parameters, can be substituted into the equation for the second unknowr, and so on. For each unknown, the most recently found estimates of the othes parameters are used. Iterations will converge to a solution of the original system for suffliently well-behaved systems.

Consider the system described by (E.4) and (E.5) above. Each parameter $T_{k}$ is given in terms of the others by

$$
T_{k}=\frac{b_{k}}{a_{k k}}-\sum_{j \neq k} \frac{a_{j k}}{a_{k k}} T_{j} .
$$

Iere $a_{k k}$ represents the $k^{\text {th }}$ diagonal element, rather than the trace, of the coeffcient riatrix. Initial estimates of all the $T_{j}$ ere made, enabling updated values to be computed. As ney yalues are found, they immedistely replace the previous estimates which had been used in the righthand-sides of the equations (E.6).

Iterative procedures such as the Gauss-Seidel methoci are particularly suited for sparse matrix inversion since the number of stored parameters and computations required depends on the number of non-zero coefficients. Given the flxed radiometer geometry, and the sky coverage having a single zenith declination, a particular pixel could be linked by measurements to pixels in at most two other regions of the sky. The number of non-zero matrix elements in the normal equations is therefore approximately proportional to the number of observed pixels, rather than to the square of the number of pixels as would be the case if the pixels were more generaliy interconnected.

A sufficient, but not necessary, condition for the iterations to converge is that the 
diagonal coefficient of each row of the coeffleient matrix dominate the other terms in the row (Caraahan et al., 1969):

$$
\left|a_{j j}\right|>\sum_{k, j=j}\left|a_{j k}\right| .
$$

If the coefficients are sufficiently well distributed (there being no $p \times q$ submatris of the $n \times n$ coefficient matrix which is a zero and for which $p+q=n$ ), the strict inequality can be relaxed to

$$
\left|a_{j j}\right| \geq \sum_{k \neq j}\left|a_{j k}\right|
$$

provided that (E.7) holds for at least one row (Hildebrand, 1956).

From the definitions in (E.4) we have $a_{j j} \equiv \sum_{k \neq j}\left|a_{j k}\right|$, not satisfying equations (E.T). Nevertheless, the map iterations converge quite rapidly for the $90 \mathrm{GHz}$ data. The RMS and the maximum change in the pixel values per iteration, taken over the entire map, decrease monotonically as iterations increase. Consecutive changes in the value of any given pixel do not necessarily decrease unless that pixel is the one having the greatest change. To provide the required additional constraint, the weighted mean of all pixel values was set to zero by addition of a constant to all pixels. The maps presented in Chapter 5 were the results of 200 iterations; the RMS change in pixel value during the $200^{\text {th }}$ iteration was on the order of tenths of $\mu \mathrm{K}$ per pixel.

\section{E.3 Jacobi Iterative Method}

The Jacobi method, like the Gauss-Seidel approach, computes updated parameter values from initial guesses or prior iteration resolts. However, the new values computed during an iteration are not used in later computations within the same iteration. Instead, all parameter values are updated simultaneously at the end of each iteration. The Jacobi method generally does not converge as fast as the Gauss-Seidel technique and was not used for the $90 \mathrm{GHz}$ maps. 


\section{E.4 Non-Iterative Method}

A differential map can be constructed from differential data without iteration by summing, with the appropriate sign, all observations of a pixel by either radiometer beam. If the radiometer output values $\Delta T_{i}$ are the differences $T_{A i}-T_{B i}$, with $T_{A i}$ and $T_{B i}$ defined as above,

then the differential map value for pixel $j$ is $\sum_{i} \Delta T_{i} \delta_{j}^{A}-\sum_{i} \Delta T_{i} \delta_{j}^{B}$. This value is equivalent to the result of one iteration of the Jacobi method if an initial value of zero is selected for all pixels. 
Appendix $\mathbf{F}$

Radiometer Parts

Component

Antenna

Batteries, gelled-electrolyte lead-acid

Coupler, directional ring

Dewar, cryogenic

Diode, detector

Diode, miser
Manufacturer and Motel

Petersen Instruments, $90 \mathrm{GHz}$ scalar feed horn

Powersonic PS-1260, PS-1265

Custom Microwave Components, WR-10 paveguide, gold-plated

Infrared Laboratories helium-cooled dewar, Model HD-3(8)

Hewlett Packard 8472B

Chip 2P8-500, GaAs, supplied by Roger Mattauch, Department of Electrical Engineering, University of Virginia, Charlottesville, VA

FETs, GaAs, for cooled IF amp Mitsubishi MGF-1412

Filter, bandpass

K \& L Microwave Model 8B120-1375/295-OP/O

Filter, notch

K \& L Microwave Model F6N45-1485.5/40-O/O

Gunn diode oscillator

Central Microwave CMF1210AE

IF amp, uncooled

Miteq Model AM-5A-0420

Mixer block

Motor, chopper

Custom Microwave Components, WR-10 waveguide, gold-plated

Globe/TRW DC. Motor 319A101-8

Globe/TRW DC. Motor 5A561-1

Develco Model 9200C:

F. W. Bell Model BH-850

Datel Intersil LPS-16

Analog Devices AD590

Baytron Co., Model 3R-50

Termination, waveguide 
Appendix G

\section{Azimuthal Anisotropy Coefficients}

For a differential radiometer having two beams at zenith angle $\theta$ which are $180^{\circ}$ apart in azimuth, the dependence of the ontput signal on azimnth angle can be used to find the dipole and quadrupole terms of a spherical-harmonic expansion e? the CBR anisotropy.

In terms of the basis functions defined in Table 5.1, the CBR temperature in the direction of unit vector $\hat{n}=\left(n_{x}, n_{y}, n_{z}\right)$ wowards (right ascension, declination) $=(\alpha, \delta)$ is given by

$$
T_{C B R}=T_{0}+\sum_{i=x, y, z} T_{i} n_{i}+\sum_{i=1,5} Q_{i} q_{i}(i)
$$

The temperature difference measured between radiometer beams $A$ and $B$ is

$$
\Delta T=T_{\hat{n}_{A}}-T_{n_{B}}=\sum_{i=x, y, z} T_{i} \cdot\left\{n_{A i}-n_{B i}\right\}+\sum_{i=1}^{5} Q_{i} \cdot\left\{g_{i}\left(\hat{n}_{, A} i-g_{i}\left(\hat{n}_{B}\right)\right\}\right.
$$

Expressing the functions $n_{i}$ and $q_{i}(\hat{n})$ of the direction vector compocents in terms of the sadiometer zenith right ascension $\alpha$ and declination $\delta$, the beam zenith angles $\theta$, and the aximuth angle $\phi$ of the direct beam $A$, the temperature difference in (G.2) becomes 


$$
\begin{aligned}
& \Delta T=(2 \sin \theta \sin \delta \cos \alpha \cos \phi+2 \sin \theta \quad \sin \alpha \sin \phi) T_{x} \\
& +(2 \sin \theta \sin \delta \sin \alpha \cos \phi-2 \sin \theta \quad \cos \alpha \sin \phi) T_{y} \\
& +\left(\begin{array}{ll}
-2 \sin \theta \cos \delta & \cos \phi
\end{array}\right) T_{2} \\
& +\left(\begin{array}{lll}
-\frac{3}{2} \sin 2 \theta \sin 2 \delta & \cos \phi
\end{array}\right) Q_{1} \\
& +(-2 \sin 2 \theta \cos 2 \delta \cos \alpha \cos \phi+2 \sin 2 \theta \sin \delta \sin \alpha \sin \phi) Q_{2} \\
& +(-2 \sin 2 \theta \cos 2 \delta \sin \alpha \cos \phi-2 \sin 2 \theta \sin \delta \cos \alpha \sin \phi) Q_{3} \\
& +(\sin 2 \theta \sin 2 \delta \cos 2 \alpha \cos \phi+2 \sin 2 \theta \cos \delta \sin 2 \alpha \sin \phi) Q_{4} \\
& +(\sin 2 \theta \sin 2 \delta \sin 2 \alpha \cos \phi-2 \sin 2 \theta \cos \delta \cos 2 \alpha \sin \phi) Q_{5} \text {. }
\end{aligned}
$$

In (G.3), the coefficients of $\cos \phi$ and $\sin \phi$ depend on the to-be-found multipole parameters $\left\{a_{j}\right\}=\left\{T_{ \pm}, T_{y}, T_{z}, Q_{1}, Q_{2}, Q_{3}, Q_{4}, Q_{5}\right\}$ as well on as the sky position and the radiometer geometry. Grouping together the $\cos \phi$ and $\sin \phi$ coefficients, we can define functions $W^{c}$ and $W^{\delta}$ by $\Delta T(\phi)=W^{c}\left(\left\{a_{j}\right\}, \alpha, \delta, \theta\right) \cos \phi+W^{\theta}\left(\left\{a_{j}\right\}, a, \delta, \theta\right) \sin \phi$. Dividing the radiometer data into intervals and fitting the data over each interval $i$ to an analogous expression $\Delta T(\phi)=$ $\left(\Delta T_{i}^{c} \pm \sigma_{i}^{c}\right) \cos \phi+\left(\Delta T_{i}^{g} \pm \sigma_{i}^{s}\right) \sin \phi$, we can form an expression for $\chi^{2}$ :

$$
\chi^{2}\{a,\}=\sum_{i}\left[\frac{W^{c}\left(\left\{a_{j}\right\}, \alpha_{i}, \delta_{i}, \theta\right)-\Delta T_{i}^{c}}{\sigma_{i}^{c}}\right]^{2}+\left[\frac{W^{s}\left(\left\{a_{j}\right\}, \alpha_{i}, \delta_{i}, \theta\right)-\Delta T_{i}^{\varepsilon}}{\sigma_{i}^{\delta}}\right]^{2} .
$$

In (G.4), $\alpha_{i}$ and $\delta_{z}$ are the arerage right ascension and declination over the $i^{\text {th }}$ interval. Minimizing $\chi^{2}$ with respect to the anisotropy dipole and quadrupole parameters $\left\{a_{j}\right\}$, as deseribed in Appendis D, determines the best-flt parameters. 


\section{Acknowledgements}

When I first got into the ballooning business, I was promptly regaled with legend and lore about various ballooning disasters of the past, including the "Lost in the Badlands" saga, the "Lost at Sea" story, and the "Great Airport Disaster". I did not expect at the time to make my own contribution to that body of technical literature. Nevertheless, I am grateful for the opportunity I've had to do this experiment and to work with those who have made it possible. Without Pbil Lubin's direction, dedication, motivation, and insight, this project would quite literally never have gotten off the ground. The experiment bas been the fruition of some of Phil's ideas, and the breeding ground for many more. A special note of thanks is due Phil's family, who endured his absence on more than a few field expeditions, and who probably thought he was done with grad student hours when he finished his own PhD. George Smoot has taught me about data, about analysis, and about what they have to do with science. He has also tried to instill in me some sense of organization. Hal Dougherty's precision mechanical design and construction was as attractive as it was functional. John Gibson designed and built most of the electronics. Both Hal and John were tolerant beyond the call of duty when yesterday's rush job became today's re-design. Tap Lum and the U.C. Radio Astromony Laboiatory were invaluable in building the GaAs FET amplifiers; George Jackson and Armi Meuti also helped build the experiment.

I especially thank Rainer Weiss and David Wilkinson for fiying, at no insignificant risk to their own experiments, the $90 \mathrm{GHz}$ radiometer on their gondolas. The skilled staff of the 
National Scientific Balloon Facility in Palestine, TX bas been competent and friendly. I also thank the Instituto de Pesqnisas Espacias in Saõ José dos Campos, SP, Brasil, for their assistance and cooperation. Ted Stubbs and the K-Division Analog Tape Center at Lawrence Livermore National Laboratory helped digitize the Brazilian telemetry data. This thesis bas been typeset. with the TeX typesetting program (Knuth, 1979).

I've enjoyed working with my fellow students: Scott "i-before-e" Friedman, Chris W-it- "e"-as-in-Edward- "b"-as-in-Baker-s-k-y Witebsky, Giovanni De Amici, Phil Melese, and Steve Levin. I also thank two of my predecessors at Princeton and Berkeley, Mark Gorenstein and Brian Corey, for their exceptionally helpful theses. Faye Mitschang and Nancy Gusack have done an admirable job keeping the Astrophysics group running; Nancy also was the one other person in Berkeley who would keep Silver Spring, MD, from going plural. The resources of the Lawrence Berkeley Laboratory bave been much appreciated and utilized, as has been the support of the U.C. Berkeley Space Sciences Laboratory. This work has been supported in part by NASA and the Department of Energy. California Space Institute (CalSpace) funding has also been particularly appreciated. The Fannie and John Hertz Foundation has supported me throughout my graduate stay at Berkeley. 


\section{References}

Alpher, R. and Herman, R., Phys. Rev. 75, 1089 (1949).

Barber, Mark, IEEE Trans. Microwave Theory Tech. MTT-15, 629 (1967).

Barrow, J. D. and Matzner, R. A., Mon. Not. R. Astr. Soc. 181, 719 (1977).

Barrow, J. D. and Turner, M. S., Nature 298, 801 (1982).

Bevington, P. R., Data Reduction and Error Analysis for the Physical Sciences, McGram-Hill, New York (1969).

Boldı, E., Comments Astrophys. 9, 97 (1981).

Bondi, H. and Gold, T., Mon. Not. R. Astr. Soc. 108, 252 (1948).

Born, M. and Wolf, E., Principles of Optics, Pergamon Press (1964).

Boughn, S. P., Fram, D. M., Partridge, R. B., Ap. J. 165, 439 (1971).

Boughn, S. P., Cheng. E. S., and Wilkinson, D. T., Ap. J. Lett. 243, L113 (1981).

Carnahan, B., Luther, H. A., and Wilkes, J. O., Applied Numerical Methods, Wiley, New York (1969).

Carr. B. J. and Rees, M. J., Nature 278, 605 (1979).

Carter, "Large Number Coincidences and the Anthropic Principle in Cosmologr," in Confrontation of Cosmological Theories with Observational Datc edited by M. S. Longair, I.A.Y. Symposium Noo. 63, D. Reidel, Dordrecht, Holland (19T々).

Ceccarelli, C., Dall'Oglio, G., Melchjorri, F., and Pietranera, L., Ap. J. 260, 484 (1982).

Cheng. E. S., Saulson, P. R., Wilkinson, D. T., and Corey, B. E., Ap. J. Lett. 232, L139 (1979).

Colc. A., Court, A, and Kantor, A. J., "Model Atmospheres," in Handbook of Geophysics and Space Environment, edited by S. L. Valley, Oftice of Aerospace Research, USAF, Cambridgc Research Lab (1965). 
Collins, C. B. and Hawking, S. W., Ap. J. 180, 317 (1973a).

Collins, C. B. and Hawking, S. W., Mon. Not. R. Astr. Soc. 162, 307 (1973b).

Conklin, E. K., Nature 222, 971 (1969).

Corey, B., Ph.D. Thesis, Princeton University (1978).

Danese, L. and De Zotti, G., Astron. Astrophys. 94, L33 (1981).

Davis, Jesse, NRAO Electronics Division Intemal Report 177 (1977).

Davis, M. and Peebles, P. J. E., Ap. J. 267, 465 (1983a).

Davis, M. and Peebles, P. J. E., Ann. Rev. Astron. Astrophys. 21, 109 (1983b).

De Vaucouleurs, G., Peters, W., Bottinelli, L., Gougenheim, L., Paturel, G., Ap. J. 248, 408 (1981).

Dicke, R. H., Nature 192, 440 (1961).

Dicke, R. H., Rev. Sci. Inst. 17, 268 (1946).

Dicke, R. H., Beringer, R., Kyhl, R. L., and Vane, A. B., Phys. Rev. 70, 340 (1946).

Dicke, R. H., Peebles, P. J. E., Roll, P. G., and Wilkinson, D. W., Ap. J. 142, 414 (1965).

Erans, G. and McLeish, C., RF Radiometer Handbook, Artech House, Inc., Dedham, MA. (197T).

Fabbri, R., Guidi, I., Melchiorri, F. and Natale, V., Phys. Rev. Lett. 44, 1563 (1980).

Fabbri, R., Guidi, I., and Natale, V., Ap. J. 257, 17 (1982).

Fall, S. M., and Jones, B. J. T., Nature 262, 467 (1976).

Fissen, D., Ph.D. Thesis, Princeton University (1982).

Fixsen, D. J., Cheng, E. S. and Wilkinson, D. T., Phys. Rev. Lett. 50, 620 (1983).

Gamow, G., Phys. Rev. 70, 572 (1946).

Gamow, G., Phys. Rev. 74, 505 (1948).

Gorenstein, M. V., Ph.D. Thesis, University of California, Berkeley (1978).

Gorenstein, M. V. and Smoot, G. F., Ap. J. 244, 361 (1981).

Guth, A. H., Phys. Rev. D 23, 347 (1981).

Harrison, E. R., Cosmology, the Science of the Universe, Cambridge Universe Press, Cambridge (1981).

Hart. L., and Davies, R. D., Nature 297, 191 (1982). 
Hawking, S., Mon. Not. R. Astr. Soc. 142, 129 (1969).

Haslam, C. G. T., Salter, C. J., Stofel, H., and Wilson, W. E., Astron Astrophys. Suppl. Ser. 47, 1 (1982).

Heiles, C., and Habing, H. J., Astron. Astrophys. Suppl. Ser. 14, 1 (1974).

Hildebrand, F. B., Introduction to Numerical Analysis, McGraw-Hill, New York (1956).

Bogan, C. J., Kaiser, N., and Rees, M. J., Phil. Trans. R. Soc. Lond. A 307, 97 (1982).

Janssen, M., Bednarczyk, S., Gulkis, S., Marlin, H., and Smoot, G. F., IEEE Trans. Ant. Prop. AP-27, 551 (1979).

Kerr, Anthony, IEEE Thans. Microwave Theory Teeh. MTT-23, 781 (1975).

Knuth, Donald E., TeX and Metafont, New Directions in Typesetting, Digital Press, Bedford, MA (1979).

Kraus, John D., Radio Astronomy, McGraw-Hill, New York (1966).

Kuhn, Thomas S., The Copernican Revolution, Planetary Astronomy in the Development of Western Thought, Harvard University Press, Cambridge, MA (1957).

Kuhn, Thomas S., The Structure of Scientific Revolutions, Chicago University Press, Chicago $(19 \div 0)$

Lubin, P., Epstein, G., and Smoot, G., Phys. Rev. Lett. 50, 616 (1983a).

Lubin, P., Melese, P., and Smoot, G., Ap. J. Lett. 273, Ljl (1983b).

Lubin, P., Fixsen, D., Cheng, E., and Epstein, G., Ap. J., submitted (1983c).

Martin. B. R., Stotistics for Physicists, Academic Press, London (1971).

Matber, J. and Kelsall, T., Physica Scripte 21, 671 (1980).

Meyer, D. H. and Jura, M., Ap. J. Lett. 276, Ll (1984).

Mise, E., Zeitschrift fïr Astrophysik 6, 1 (1933).

Misner, C. W., Thorne, K. S., and Wheeler, J. A., Gravitation, W. H. Freeman and Co., San Francisco (1973).

Misner, C. W., Ap. J. 151, 431 (1968).

NAS_ Goddard Space Fligbt Center, Interim Study Report for Cosmic Background Explorer, p. $48(1977)$.

Owens, D. K., Muehlner, D. J., and Weiss, R., A p. J. 231, 702 (1979).

Partridge, R. P. and Wilkinson, D. T., Phys. Rev. Lett. 18, 55 i (196i). 
Peebles, P. J. E., Physical Cosmology, Princeton University Press, Princeton, New Jersey (I97I).

Peebles, P. J. E. and Wilkinson, D. T., Phys. Rev. 174, 2168 (1968).

Peebles, P. J. E., Ap. J. Lett. 243, L119 (1981).

Peebles, P. J. E., Ap. J. 259, 442 (1982).

Penzias, A. A. and Wilson, R. W., Ap. J. 142, 419 (1965).

Press, W. H., "Galaxies may bc Single Particle Fluctuations from an Early False-Vacuum Era," in Cosmology and Particles, edited by Audouze et al., Proreedings of the Sixteenth Rencontre de Moriond Astropbysics Meeting, Singapore National Printers (1981).

Rees, M. J., Ap. J. Lett. 153, L1 (1968).

Roll, P. G. and Hiikinson, D. W., Phys. Rev. Lett. 16, 405 (1966).

Roll, P. G. and Wilkinson, D. W., Annals of Physics 44, 289 (1967).

Rubin, V. C., Thonnard, N., Ford, W. K., and Roberts, M. S., Astron. Jaur, 81, 719(1976).

Sandage, A. and Tammann, G. A., Ap: J. Lett. 207, L1 (1976).

Sciama, Dennis, Modern Cosmology, Cambridge, Universicy Press. Cambridge (1971).

Silk, Joseph, The Big Bang, W. H. Freeman and Company, San Francisco (1980).

Silk, J. and Wilson, M., Ap. J. Lett. 244, L37 (1981).

Smoot, G., De Amici, G., Fricdman, S., Witebsky, C., Mandolesi, R.. Partridge, B., Sironi, G., Danese, L, and De Zotti, G., Phys. Rev. Lett. 51, 1099 (1983).

Smoot, G. F., Gorenstein, M. V., and Muller, R. A., Phys. Rev. Lett. 39, 898 (1977).

Smoot, G. F. and Lubin, P. M., Ap. J. Lett. 234, L83 (1979).

Ulaby, Faw az T., Moore, Richard K., and Fung, Adrias K., Microwave Remote Sensing, Active and Passive, Vol. 1, Addisin-Wesley, London (1981).

Weaver, H., and Williams, D. R. W., Astron. Astrophys. Supp. Ser. 8, 1 (1973).

Wejnreb, Sander and Kerr, Anthony, IEEE J. Solid-State Circuits SC-8, 58 (1973).

Weinberg, S., Gravitation and Cosmology: Principles and Applications of the General Theory of Relativity, John Wiley and Sons, New York (1972).

Weinberg. S., The First Three Minutes, Basic Books, New Yorh (197i).

Wejss. R. W., Ann. Rev. Astron. Astrophys. 18, 489 (1980).

Williams, D. R. and Lum, W., Microwave Journal 23. No. 11. 73. (October 1980). 
Wilson, R. W., Science 205, 866 (1979).

Wilson, R. W. and Penzias, A. A., Science 156, 1100 (1967).

Witebsky, C., Univ. Cal. Berkeley Space Sciences Laboratory Astrophysical Note 374 (COBE Memo \# 5018), unpublished (1978).

Woody, D. P. and Richards, P. L., Phys. Rev. Lett. 12, 925 (1979).

Yahil, A., Tammann, G. A., and Sandage, A., Ap. J. 217, 903 (1977).

Yang, J., Schramm, D., Steigman, G., and Rood, R., Ap. J. 227, 697 (1979). 
This report was done with suppore from the Department of Energy. Any conclusions or opinions expressed in this report represent solely those of the author(s) and not necessarily those of The Regents of the University of California, the Lawrence Berkeley Laboratory or the Department of Encrgy.

Reference 10 a company or product name does not imply approval or recommendation of the product by the University of California or the U.S. Department of Energy to the exclusion of others that may be suitable. 